\title{
Depressiv-ängstliche Störungen bei somatischen Krankheiten 62
}

\author{
H.-P. KAPFHAMMER
}

\author{
$62.1 \quad$ Einleitung 1559 \\ 62.1.1 Klassifikation 1560 \\ 62.1.2 Klinische Bedeutung 1561 \\ 62.2 Epidemiologie 1562 \\ 62.3 Ätiopathogenese 1563 \\ 62.3.1 Psychologische Bedingungsfaktoren 1564 \\ 62.3.2 Biologische Bedingungsfaktoren 1565 \\ 62.3.3 Soziale Bedingungsfaktoren 1567 \\ 62.3.4 Bedingungsgefüge am Beispiel \\ „Krebserkrankung“ 1568 \\ 62.4 Symptomatologie 1571 \\ 62.5 Verlauf und Prognose 1573 \\ 62.6 Diagnostik und Differentialdiagnose 1574 \\ 62.7 Therapie 1575 \\ 62.7.1 Psychologische Ansätze 1575 \\ 62.7.2 Biologische Ansätze 1576 \\ 62.7.3 Einsatz von Antidepressiva \\ bei definierten internistischen Erkrankungen 1580 \\ Literatur 1587
}

$\mathrm{D}$ epressiv-ängstliche Störungen sind bei den unterschiedlichen somatischen Erkrankungen häufig. Sie sind nicht nur als Reaktion auf die Situation der Erkrankung zu verstehen, sondern in ein komplexes Bedingungsgefüge eingebettet. Sie sind besonders häufig bei Erkrankungen, die das Zentralnervensystem oder endokrine Regulationsysteme direkt betreffen. Außerdem besteht ein enger Zusammenhang zur Chronizität, Schwere und Prognose der Erkrankung. Eigenständige Effekte von diversen pharmakologischen Substanzgruppen sind wahrscheinlich.

Beim Verlauf somatischer Erkrankungen sind negative Auswirkungen koexistenter depressiv-ängstlicher Störungen auf die psychosoziale Adaptation, das medizinische Inanspruchnahmeverhalten, die Morbidität und Mortalität zu beachten. Psychotherapeutische Verfahren besitzen einen wichtigen Stellenwert im Gesamtbehandlungsplan. Differenzierte psychopharmakologische Strategien sind für die einzelnen somatischen Erkrankungen unter Kenntnis wichtiger Nebenwirkungen, der Interaktionen mit Internistika und der pathophysiologischen Grundbedingungen der somatischen Erkrankung verfügbar.
62.1

\section{Einleitung}

Ängste und Depressionen sind sehr häufige Begleitumstände körperlicher Krankheiten. Im psychiatrischen Konsiliardienst stellen sie eine bedeutsame Hauptgruppe der Überweisungsgründe zur konsiliarischen Untersuchung und Mitbehandlung dar (s. Kap. 72). Aufgrund der besonderen Häufigkeit sowohl von somatischen Erkrankungen einerseits, von depressiven und Angststörungen andererseits in der Allgemeinbevölkerung mag ein zufälliges Zusammentreffen nicht überraschen. Diesem Sachverhalt kommt mit steigendem Lebensalter eine zunehmende Bedeutung zu. Gerade im höheren Lebensalter können typische Depressionen, aber auch klinisch relevante Ängste erstmalig im Kontext zunehmend häufiger werdender somatischer Erkrankungen ausgelöst werden (Satlin 1998). Jenseits einer zufälligen epidemiologischen Koexistenz muß aber auch stets ein komplexes, interagierendes Bedingungsgefüge zwischen somatischen Krankheiten, Therapien und psychischen Störungen angenommen werden. Koexistenz bzw. Komorbidität von körperlicher Krankheit und psychischer Störung verweisen hierbei grundsätzlich auf 4 Hauptkategorien:

Zufällige Koinzidenz. Trotz eines fehlenden kausalen Zusammenhangs zwischen psychischer Störung und körperlicher Krankheit bedeutet die zusätzliche psychische Störung in aller Regel eine Komplizierung für das Management der körperlichen Krankheit.

Gemeinsame Ursache für psychische Störung und körperliche Krankheit. Aus einem belastenden negativen Lebensereignis können prinzipiell beide Erkrankungen resultieren. In der individuellen Sicht kann der Verarbeitung eines bestimmten Lebensgeschickes mitunter ein höherrangiger Stellenwert eingeräumt werden als der Bedeutung der körperlichen Krankheit und folglich zu einem stärkeren psychosozialen Streß führen.

Psychische Störung verursacht körperliche Krankheit. Klinische Angst und Depression beeinflussen die 
Auslösung, Exazerbation und Aufrechterhaltung einer körperlichen Krankheit. Sie komplizieren den Krankheitsverlauf signifikant.

Körperliche Krankheit verursacht psychische Störung. Eine körperliche Krankheit verursacht bei einer prädisponierten Person eine psychische Störung z.B. Angst oder Depression über einen biologischen Mechanismus und/oder eine psychologische Reaktion. Die psychische Störung kann hierbei de novo entstehen, entweder bei einer vulnerablen Persönlichkeit durch die Krankheit als bedeutsamen psychosozialen Stressor oder durch krankheitsimmanente pathophysiologische Prozesse ausgelöst werden. Selbstverständlich kann auch eine Person mit einer positiven psychiatrischen Anamnese im Kontext der Erkrankung durch die hier wirksamen psychosozialen und/oder biologischen Einflußfaktoren leichter ein Rezidiv erleiden.

\subsection{1}

\section{Klassifikation}

Allein diese kurze Skizzierung veranschaulicht, daß eine vorrangig auf deskriptive Kategorien fußende Klassifikation sich großen konzeptuellen Problemen gegenübersieht, dem klinischen Stellenwert von depressiv-ängstlichen Störungen bei somatischen Erkrankungen gerecht zu werden (Kapfhammer 2001). Dies spiegelt sich u. a. in den sehr divergierenden diagnostischen Konzepten in der Geschichte der psychiatrischen Klassifikationssysteme wieder, die auf diesen Störungsbereich angewendet wurden und werden.

\section{Konzept der sekundären Depression}

So zielte z. B. das Konzept der „sekundären Depression“ (Robbins u. Guze 1972) bei einem depressiven Syndrom zunächst auf die Trennung von primären und sekundären affektiven Störungen. Die Unterscheidung erfolgte hierbei über 2 Kriterien:

- zunächst über eine zeitliche Bestimmung der depressiven Indexepisode in der chronologischen Entwicklung einer affektiven Störung (z. B. unipolar vs. bipolar) oder

- in der Abfolge einer anderen psychiatrischen Störung (z. B. Schizophrenie, Alkoholismus), die der depressiven Indexepisode zeitlich vorausgeht.

Dieses Konzept implizierte also keine Differenzierung nach dem Schweregrad eines depressiven Syndroms, berücksichtigte auch nicht vorliegende oder fehlende bedeutsame Lebensereignisse oder spezielle Symptommuster, sondern lediglich die zeitliche Reihenfolge.

Klerman (1981) plädierte nun in Anlehnung an Robbins u. Guze (1972) für eine eigenständige diagnostische Kategorie einer „sekundären Depression bei einer körperlichen Krankheit bzw. bei bestimmten medikamentösen Therapien“. Er hatte hierbei die besondere zeitliche Verwobenheit, aber wohl auch eine spezielle symptomatische Ausgestaltung depressiver Syndrome im Kontext somatischer Erkrankungen im Auge. Ähnlich argumentierte Cassem (1990) für eine gesonderte Betrachtung von „Depression und Angst als sekundären Störungen bei einer körperlichen Krankheit“.

\section{Klassifikation durch ICD und DSM}

Weder die ICD-10 noch das DSM-III-R und DSM-IV kennen eine konzeptuelle Unterscheidung in „primär und sekundär" mit den zeitlichen und inhärent kausalen Implikationen (Morrison u. Kastenberg 1997). Die Diagnose einer „Major Depression“ wird sowohl in der ICD-10 als auch im DSM-III-R bei körperlichen Krankheiten infolge des Ausschlußkriteriums einer möglichen „organischen Bedingtheit" sogar erschwert.

\section{Major Depression vs. organische affektive Störung}

Die Alternativdiagnose einer „organischen affektiven Störung" wiederum kann aber schon bei 2 Zusatzsymptomen aus der geforderten Symptomliste einer Major Depression gestellt werden. Die Validität einer Unterscheidung von Major Depression vs. organischer affektiver Störung muß deshalb gerade für den konsiliarpsychiatrischen Bereich stark bezweifelt werden. Unabhängig davon, daß bei einer somatischen Grunderkrankung im Einzelfall der Beweis für eine organische Bedingtheit der affektiven Störung nur schwerlich vollständig $\mathrm{zu}$ führen ist, erscheint eine streng an den klinischen Symptomen orientierte Beschreibung auf der Achse I sowie dann eine Identifikation von relevanten organischen Faktoren auf der Achse III im DSM-System als weitaus sinnvoller (Fogel 1990).

Bei somatischen Erkrankungen muß zudem festgehalten werden, daß die körperlichen Symptome wie Appetit- und Schlafstörungen oder Energieverlust zunächst nur wenig über das eventuelle Vorliegen einer Depression aussagen, die kognitiven und affektiven Symptome hingegen eine gute Diskriminationskraft besitzen (Fava u. Molnar 1987). Von Ammon Cavenough (1984) riet, bei körperlich kranken Patienten die somatischen Symptome nur zur Stützung der Diagnose einer Major Depression heranzuziehen, wenn sie ernster Natur, unverhältnismäßig im Vergleich zum vorliegenden Krankheitsprozeß erscheinen und mit den affektivkognitiven Symptomen zeitlich korrelieren (s. auch Abschn. 62.6)

\section{Angststörung und Anpassungsstörung}

Ganz analoge Probleme stellen sich bei somatisch kranken Patienten in der ICD-10 und im DSM-III-R auch für die Diagnosestellung von „Angststörungen“ einerseits, von „organischen Angststörungen“ andererseits. Eine 
ähnliche diagnostische Haltung wie im Fall der Depression empfiehlt sich auch hier.

Die verbleibende diagnostische Kategorie einer $A n$ passungsstörung mit depressiver und/oder ängstlicher Verstimmung kann mit führenden Symptomen einer depressiven Weinerlichkeit und Hoffnungslosigkeit sowie einer ängstlichen Nervosität für jene Patienten reserviert werden, bei denen die affektiven bzw. Angstsymptome den für eine Major Depression bzw. eine Angststörung geforderten diagnostischen Kriterien nicht voll genügen.

\section{Dysthymie vs. Minor Depression}

Das diagnostische Konzept der Dysthymie betrifft wiederum ein chronisches depressives Syndrom von in der Regel mäßiger Intensität, d.h. die vollen Kriterien einer Major Depression sind nicht erfüllt. Cassem (1990) favorisierte hierfür den Terminus einer „Minor Depression" und plädierte dafür, die explizite Forderung einer mindestens über 2 Jahre bestehenden dysphorischen Stimmungslage bei somatisch kranken Patienten aufzugeben.

\section{Klassifikation durch DSM-IV}

Auch im DSM-IV sind die diagnostischen Probleme für diesen konsiliarpsychiatrisch sehr relevanten Störungsbereich letztlich nicht gelöst. Das DSM-IV weist im Vergleich zur ICD-10, aber auch in Abgrenzung zum Vorläufer DSM-III-R 2 wesentliche Neuerungen auf.

\section{Zusammenhänge statt Dichotomie}

Zum einen hat es die implizite traditionelle Orientierung an „organisch vs. funktionell-reaktiv" aufgrund moderner Erkenntnisse der Neurowissenschaften als eine überholte Dichotomisierung fallen gelassen. So kennt es weder eine „organische affektive Störung“ noch eine „organische Angststörung". Statt dessen führt es sowohl eine „affektive Störung aufgrund eines medizinischen Krankheitsfaktors“ bzw. eine „substanzinduzierten affektiven Störung“ als auch eine „Angststörung aufgrund eines medizinischen Krankheitsfaktors" bzw. eine „substanzinduzierten Angststörung“. Somatischer Status, Anamnese, Laborbefunde und/oder andere medizinische Untersuchungsparameter müssen die affektive Störung bzw. Angststörung als eine direkte körperliche Folge (in einem zeitlichen und kausalen Sinne) ausweisen.

Auch hier existiert dasselbe Problem eines überzeugenden Nachweises der „organischen Bedingtheit bzw. substanzbezogenen Induktion" wie bei den diagnostischen Vorläuferkonzepten der „organischen psychischen Störungen " in DSM-III-R bzw. ICD-10. Und diese diagnostischen Kategorien können dann in eine Aporie einmünden, wenn offenkundig nur ein sehr variabler Prozentsatz von Patienten mit definierten somatischen
Erkrankungen oder unter bestimmten pharmakologischen Behandlungen mit depressiven oder Angststörungen reagiert, also erneut der Stellenwert von Dispositions- bzw. Vulnerabilitätsfaktoren in der ätiologischen Diskussion aufscheint (Whitlock 1982).

\section{Label statt Kategorie}

Zum anderen hält das DSM-IV eine sehr breite diagnostische Gruppierung bereit, die im DSM-III-R nur knapp eine zeitliche Beziehung von psychologischen Faktoren zur Auslösung und Exazerbation eines medizinischen Zustands skizzierte. Nunmehr fokussiert ein diagnostisches Label, weniger eine diagnostische Kategorie im engeren Sinne auf komplexe interaktive Effekte von psychologischen Faktoren auf körperliche Krankheitsbedingungen. In dieser Gruppierung, die mit dem Titel Psychologische Faktoren, die einen medizinischen Krankheitsfaktor beeinflussen überschrieben ist, können psychologische Faktoren

- als eine „psychische Störung“ der Achse I und II (z.B. Major Depression, Borderline-Persönlichkeitsstörung),

- als „psychische Symptome“, die noch unter der Schwelle einer psychischen Störung liegen (z.B. Angst, die ein Asthma bronchiale verstärkt),

- als „Persönlichkeitszüge oder Copingstile“ (z. B. TypA-Verhalten bei einer koronaren Herzerkrankung),

- als „maladaptive Gesundheitsverhaltensweisen“ (z. B. Rauchen bei kardiovaskulären Erkrankungen) oder aber

- als „streßinduzierte Reaktionen“ (z. B. psychosoziale Stressoren, die einen Hypertonus beeinflussen) konzipiert werden. Die Effekte auf einen Krankheitszustand müssen nachweisbar sein. Die medizinischen Krankheitsfaktoren selbst werden näher auf der Achse III ausgeführt (Stoudemire u. Hales 1991).

\section{Fazit}

Die im vorliegenden Kapitel erörterten depressivängstlichen Störungen bei somatischen Krankheiten betreffen also komplexe wechselseitige Interaktionen zwischen Psyche und Soma. Im Verständnis von DSMIV werden vorrangig die diagnostischen Kategorien von „depressiven Störungen“ bzw. „Angststörungen aufgrund eines medizinischen Krankheitsfaktors" bzw. „aufgrund einer Substanzinduktion“ zum einen, von "psychologischen Faktoren, die einen medizinischen Krankheitsfaktor beeinflussen“, zum anderen berührt.

\subsection{2 \\ Klinische Bedeutung}

Im Behandlungssetting eines internistischen Krankenhauses wird allenfalls die Hälfte der an depressiven oder Angststörungen leidenden Patienten in ihrer psychia- 
trischen Problematik erkannt (Fifer et al. 1994; Schulberg et al. 1985). Dies scheint relativ unabhängig von der Schwere der psychischen Störung zu sein (Feldman et al. 1987; Perez-Stable et al. 1990; Sliman et al. 1992). Arztbezogene, patientenbezogene und strukturelle Faktoren dürften diese klinische Realität der Unterdiagnostiziertheit und -therapiertheit bestimmen (s. Übersicht).

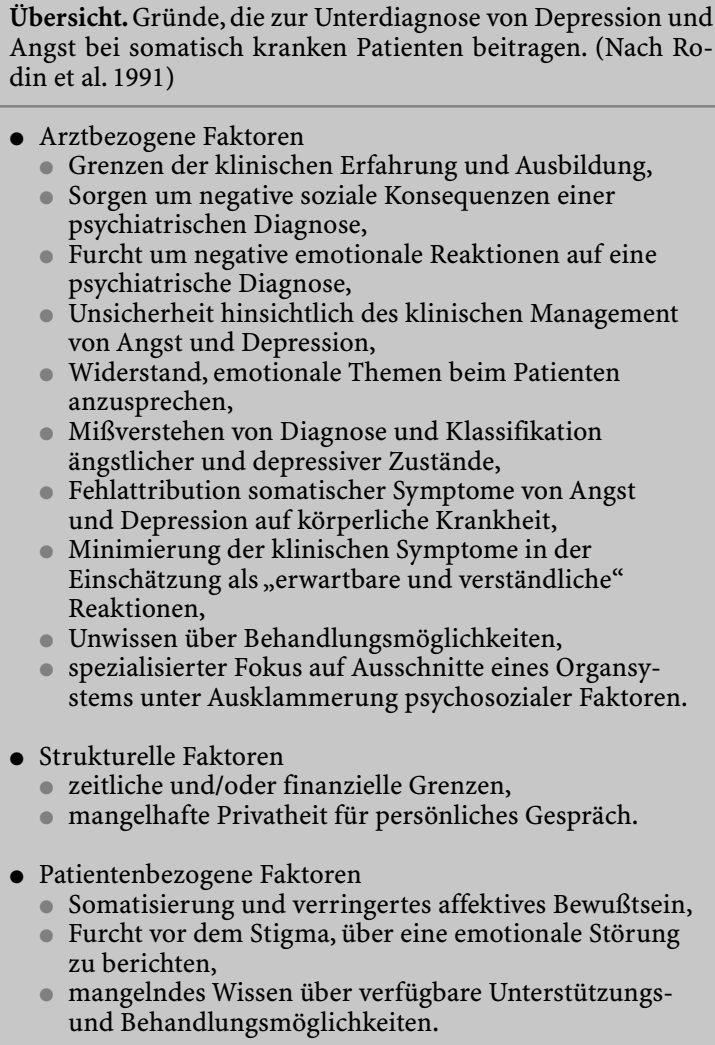

Arztbezogene Faktoren

- Grenzen der klinischen Erfahrung und Ausbildung,

- Sorgen um negative soziale Konsequenzen einer psychiatrischen Diagnose,

- Furcht um negative emotionale Reaktionen auf eine psychiatrische Diagnose,

- Unsicherheit hinsichtlich des klinischen Management von Angst und Depression,

- Widerstand, emotionale Themen beim Patienten anzusprechen,

- Mißverstehen von Diagnose und Klassifikation ängstlicher und depressiver Zustände,

- Fehlattribution somatischer Symptome von Angst und Depression auf körperliche Krankheit,

- Minimierung der klinischen Symptome in der Einschätzung als ,erwartbare und verständliche“ Reaktionen,

- Unwissen über Behandlungsmöglichkeiten,

- spezialisierter Fokus auf Ausschnitte eines Organsystems unter Ausklammerung psychosozialer Faktoren.

- Strukturelle Faktoren

zeitliche und/oder finanzielle Grenzen,

- mangelhafte Privatheit für persönliches Gespräch.

- Patientenbezogene Faktoren

- Somatisierung und verringertes affektives Bewußtsein,

- Furcht vor dem Stigma, über eine emotionale Störung zu berichten,

- mangelndes Wissen über verfügbare Unterstützungsund Behandlungsmöglichkeiten.

Patienten werden infolge ihrer nicht beachteten affektiven und Angststörungen signifikant häufiger medizinisch-diagnostischen Prozeduren unterzogen, ihre stationären Verweildauern sind bedeutsam länger (Beitman et al. 1989; Katon et al. 1986). Andererseits werden internistische und chirurgische Patienten nicht selten auch ohne konsiliarpsychiatrische Beurteilung mit Psychopharmaka der unterschiedlichsten Substanzklassen behandelt.

Depressiv-ängstliche Affektlabilität und Schmerzzustände stellen Hauptindikationen dar. Eine Tendenz zur Behandlung mit erstaunlich niedrigen Dosierungen, seltene Dosisanpassungen im Hinblick auf Therapieeffekte oder Nebenwirkungen sowie eine unkontrollierte Fortführung der einmal etablierten Medikation auch über den stationären Aufenthalt hinaus fallen auf (Callies u. Popkin 1987).

\section{2 \\ Epidemiologie}

Exakte Häufigkeitsangaben über depressiv-ängstliche Störungen bei Patienten mit somatischen Erkrankungen sind infolge heterogener diagnostischer Konzepte und Erhebungsinstrumente, unterschiedlicher Patientensamples, divergierender Behandlungssettings (ambulante vs. stationäre Versorgung) und nicht weiter spezifizierter Stadien im Krankheitsverlauf nur schwer aus der vorliegenden Literatur abzuleiten.

\section{Major depression}

Während etwa 2-4\% der Allgemeinbevölkerung an einer Major Depression leiden, erhöht sich bei Personen mit einer körperlichen Grunderkrankung dieses Risiko hinsichtlich affektiver Störungen, aber auch hinsichtlich des Mißbrauchs von Alkohol oder psychotropen Substanzen signifikant. Bestimmte chronisch-medizinische Probleme wie rheumatoide Arthritis, Karzinom-, Lungen-, Herz- und neurologische Erkrankungen scheinen hierfür besonders zu prädisponieren (Wells et al. 1988). Die Prävalenz einer Major Depression steigt bei ambulant behandelten Patienten auf ca. 9\% an und kann bei Patienten in stationärer Therapie Häufigkeiten von über $20 \%$ erreichen (Katon u. Sullivan 1990).

\section{Depressive Störungen}

Die Prävalenzzahlen für depressive Störungen bei einzelnen somatischen Krankheiten weisen eine hohe Spannbreite auf. Sie hängen nicht zuletzt von der Art der Diagnosestellung ab. Sie sind in den Studien am niedrigsten, die ein strukturiertes klinisches Interview einsetzten. Sie sind am höchsten in den Untersuchungen, die methodisch auf psychologischen Befragungen oder Selbsteinschätzungskalen beruhten. Tabelle 62.1 zeigt eine Auswahl von Studien, die sich auf strukturierte klinische Interviews mit definierten Diagnosekriterien bei somatisch kranken Patienten in stationärer Behandlung stützten. Die auch hier beträchtlich schwankenden Häufigkeitsangaben sind u.a. auf unterschiedliche Schweregrade und Prognosen zurückzuführen.

\section{Angststörungen}

Unter ambulant versorgten Patienten mit gewöhnlichen körperlichen Krankheiten (z.B. Hypertonus, Diabetes mellitus, koronare Herzkrankheit) hatten 15-18\% aktuelle Angsterkrankungen. Hierunter imponierten Panikstörung mit 1,0-1,7\% und generalisierte Angststörung mit 10,4-12,4\% (Sherbourne et al. 1996).

Die Prävalenzzahlen für aktuelle Angststörungen bei Personen mit chronischen Erkrankungen in der Allgemeinbevölkerung zeigen eine differentiell ausgeprägte Assoziation (Wells et al. 1989):

- keine medizinische Bedingung: 6,0 $\pm 0,6 \%$,

- rheumatoide Arthritis 11,9 $\pm 2,6 \%$, 
Tabelle 62.1. Prävalenz depressiver Störungen bei Patienten mit internistischen/neurologischen Erkrankungen

\begin{tabular}{|c|c|c|c|c|c|}
\hline Krankheit & $\begin{array}{l}\text { Anzahl } \\
\text { (n) }\end{array}$ & Instrument & Diagnose & $\begin{array}{l}\text { Prävalenz } \\
(\%)\end{array}$ & Autoren \\
\hline Allgemein internistisch & 294 & DIS \& CESDS & Alle depressiven Störungen & 9,2 & Schulberg et al. (1985) \\
\hline Allgemein internistisch & 809 & DIS & $\mathrm{MDE} / \mathrm{DD}$ & $5,0 / 3,7$ & von Korff et al. (1987) \\
\hline Allgemein internistisch & 294 & DIS & MDE & 7,1 & Coulehan et al. (1988) \\
\hline Neurologisch/internistisch & 128 & DIS, SADS & $\mathrm{MDE}$ & 12,0 & Koenig et al. (1988) \\
\hline Niereninsuffizienz & 99 & DIS & $\mathrm{MDE} / \mathrm{DD}$ & $8,1 / 6,1$ & Craven et al. (1987) \\
\hline Niereninsuffizienz & 124 & SADS & MDE/MinD & $6,5 / 17,7$ & Hinrichsen et al. (1989) \\
\hline Post-Stroke & 103 & PSE & $\mathrm{MDE} / \mathrm{DD}$ & $27,0 / 20,0$ & Robinson et al. (1983) \\
\hline Parkinson & 105 & $\begin{array}{l}\text { Strukturiertes } \\
\text { klinisches Interview }\end{array}$ & MDE/MinD & $21,0 / 20,0$ & Starkstein et al. (1990) \\
\hline Onkologisch-stationär & 62 & Klinisches Interview & MDE/som. exklusiv & $42,0 / 24,0$ & Bukberg et al. (1984) \\
\hline Onkologisch-stationär & 215 & Klinisches Interview & $\begin{array}{l}\text { MD-uni/-bipolar/ } \\
\text {-atypisch/ADDM }\end{array}$ & $\begin{array}{l}3,7 / 0,5 \\
1,4 / 12,1\end{array}$ & Derogatis et al. (1983) \\
\hline $\begin{array}{l}\text { Diabetes mellitus vor } \\
\text { Pankreastransplantation }\end{array}$ & 75 & DIS & $\begin{array}{l}\text { MDE (L-Zeit)/(6 Mo) } \\
\text { DD (L-Zeit) }\end{array}$ & $\begin{array}{l}24,0 / 10,7 \\
4,0\end{array}$ & Popkin et al. (1988) \\
\hline Akuter Myokardinfarkt & 100 & $\begin{array}{l}\text { Standardisiertes } \\
\text { klinisches Interview }\end{array}$ & Depr. Neurose (ICD-8) & 18,0 & Lloyd u. Cawley (1978) \\
\hline Gastroenterologisch & 23 & $\begin{array}{l}\text { Klinisches Interview } \\
\text { nach Paykel }\end{array}$ & MDE & 43,5 & Fava et al. (1985) \\
\hline Rheumatoide Arthritis & 137 & DIS & $\mathrm{MDE} / \mathrm{DD}$ & $16,8 / 40,1$ & Frank et al. (1988) \\
\hline $\begin{array}{l}\text { Kandidaten für Herztrans- } \\
\text { plantation }\end{array}$ & 68 & $\begin{array}{l}\text { Semistrukturiertes } \\
\text { klinisches Interview }\end{array}$ & MDE & 14,7 & Maricle et al. (1989) \\
\hline
\end{tabular}

DIS Diagnostic Interview Schedule; CESDS Center for Epidemiologic Studies Depression Scale; MDE Major Depression; $D D$ dysthyme Störung; MinD Minor Depression (RDC); SADS Schedule for Affective Disorders and Schizophrenia; PSE Present State Examination; $A D D M$ Anpassungsstörung mit ängstlicher/depressiver Verstimmung; L-Zeit Lebenszeitprävalenz; 6 Mo Sechsmonatsprävalenz.

- Diabetes mellitus: $15,8 \pm 6,1 \%$,

- Herzkrankheit: $21,0 \pm 5,7 \%$,

- chronische Lungenerkrankung $10,0 \pm 2,5 \%$,

- Bluthochdruck: $12,1 \pm 3,0 \%$.

In einer frühen Studie, die sich exklusiv auf Angststörungen bei stationär behandelten Patienten eines Allgemeinkrankenhauses konzentrierte, fanden Schwab et al. (1966) bei $20 \%$ der Patienten mäßige bis schwere Angstsymptome. Weitere Studien zeigten bei stationären somatisch kranken Patienten eine Häufigkeit von 6-11\% für Angststörungen (Deshpande et al. 1989; Feldman et al. 1987; Maguire et al. 1974).

\section{3}

\section{Ätiopathogenese}

Depressive und Angststörungen im Kontext somatischer Erkrankungen sind multifaktoriell bedingt (Kapfhamer 2002). Eine ätiopathogenetische Diskussion verweist zunächst auf die grundlegenden Ausführungen, die in den Darstellungen der Einzelstörungen erfolgen (s. Kap. 49 und 52). Weitere ätiopathogenetische Aspekte müssen sich aber auch mit den spezifischen Gegebenheiten einer bestimmten somatischen Krankheit und den jeweils indizierten therapeutischen Maßnahmen befassen. Psychologisch-emotionale, biologische und soziale Einflußfaktoren können prinzipiell unterschieden werden (s. Übersicht).

Übersicht. Einflußfaktoren auf das Risiko einer emotionalen Störung bei einer somatischen Krankheit. (Nach Mayou u. Sharpe 1995)

- Art der körperlichen Krankheit:

- biologische Faktoren (ZNS-Krankheiten, systemische Krankheiten).

- Krankheitsvariablen:

organpathologische Diagnose,

- anatomische Lokalisation,

- Verlauf (akut, chronisch, rezidivierend, progredient, rekonvaleszent),

- Schweregrad (Lebensbedrohung, Symptomausprägung, Behinderung, Entstellung),

- Funktionsverlust, Selbstwert.

- Behandlungsmodalitäten:

- Erfolgschancen,

- Qualität der Aufklärung/Anleitung,

- Nebenwirkungen,

- Anforderungen an Selbstfürsorge.

- Patientenbezogene Variablen:

- biologische Vulnerabilität,

- Persönlichkeit,

- psychologische Vulnerabilität,

- soziale Vulnerabilität/protektive Faktoren,

- andere psychosoziale Stressoren.

- Soziale Konsequenzen der Erkrankungen:

- Arbeit,

- Familie, soziale Beziehungen. 


\subsection{1}

\section{Psychologische Bedingungsfaktoren}

An grundlegenden psychologischen Bedingungsfaktoren sollte zunächst bedacht werden (Lipowski 1975; Schmale 1979):

- Jede akute Erkrankung unterbricht die gewohnte psychosoziale Lebensführung einer Person und kann diverse Ängste vor körperlichem Leiden, Behinderung, Abhängigkeit, persönlichen Verlusten usw. auslösen.

- Jede chronische Erkrankung geht mit einem bestimmten Grad an Irreversibilität der körperlichen Integrität, an körperlicher Funktionseinbuße und Verlust leiblichen Wohlbefindens einher und ist mit einer mehr oder weniger ausgeprägten psychosozialen Behinderung assoziiert.

- Akute wie chronische Abschnitte einer Erkrankung stellen hohe spezielle Anforderungen an die innerseelische Verarbeitung und das Copingverhalten eines Individuums und bedingen ein komplexes emotionales Erleben. Aber auch die Zeit einer Rekonvaleszenz nach einer Erkrankung kann von affektiven Prozessen bestimmt werden, die einer Trauer entsprechen.

\section{Umgang mit Bedrohung und Verlust}

In Abhängigkeit von der Art des Krankheitsprozesses, der Akuität vs. Chronizität der Krankheitsstadien, aber auch der Prognose und Aussicht auf Therapie spielen in den persönlichen Bedeutungen einer Erkrankung grundlegende Themen von Bedrohung und Verlust eine entscheidende Rolle. Während „Bedrohung“ in der Erlebniskonfrontation eher mit Angstaffekten assoziiert ist, stellt „Verlust“ den entscheidenden Ausgangspunkt für Trauer und depressive Affekte dar. Beide existentiellen Grunderfahrungen sind aber nicht polar gegenübergestellt, sondern können fließend ineinander übergehen.

Abbildung 62.1 zeigt schematisch Aspekte der psychologischen Reaktion auf eine somatische Krankheit.

Die Primärpersönlichkeit eines individuellen Patienten, sein Selbstverständnis in einem bestimmten Lebensabschnitt, seine kognitiv-affektiven Prädispositionen, aber auch intrapsychischen Konflikte beeinflussen signifikant die persönlichen Bedeutungen einer Erkrankung. Die Auseinandersetzung mit der Erkrankung beginnt mit der Herausforderung durch die individuellen Bedeutungen dieser Krankheit. Copingstile können handlungs-, affekt- und kognitionsbezogen sein und zu unterschiedlich günstigen Anpassungen führen (Schüßler 1998).

\section{Psychodynamik}

Angstkonflikte. Psychodynamisch kommt den einzelnen durch die Krankheitserfahrung ausgelösten Angstkonflikten eine spezielle Bedeutung zu. Hierunter imponieren Themen wie Bedrohung der narzißtischen und körperlichen Integrität, Furcht vor Verstümmelung oder Vitalitätsverlust, Furcht vor körperlichem Kontrollverlust, Furcht vor Autonomieverlust, Todes- und Trennungsangst (Derogatis u. Wise 1989). Diese hauptsächlichen Angstkomponenten bei schwerwiegenden körperlichen Erkrankungen stellen genuine psychodynamische Herausforderungen dar. Sie können sich aber auch mit lebensgeschichtlich ungelösten innerseelischen Konflikten oder lebenssituativen Entwicklungsaufgaben verknüpfen, die durch die Krankheitserfah-
Abb. 62.1.

Psychologische Reaktion auf eine somatische Krankheit. (Mod. nach Rodin et al. 1991)

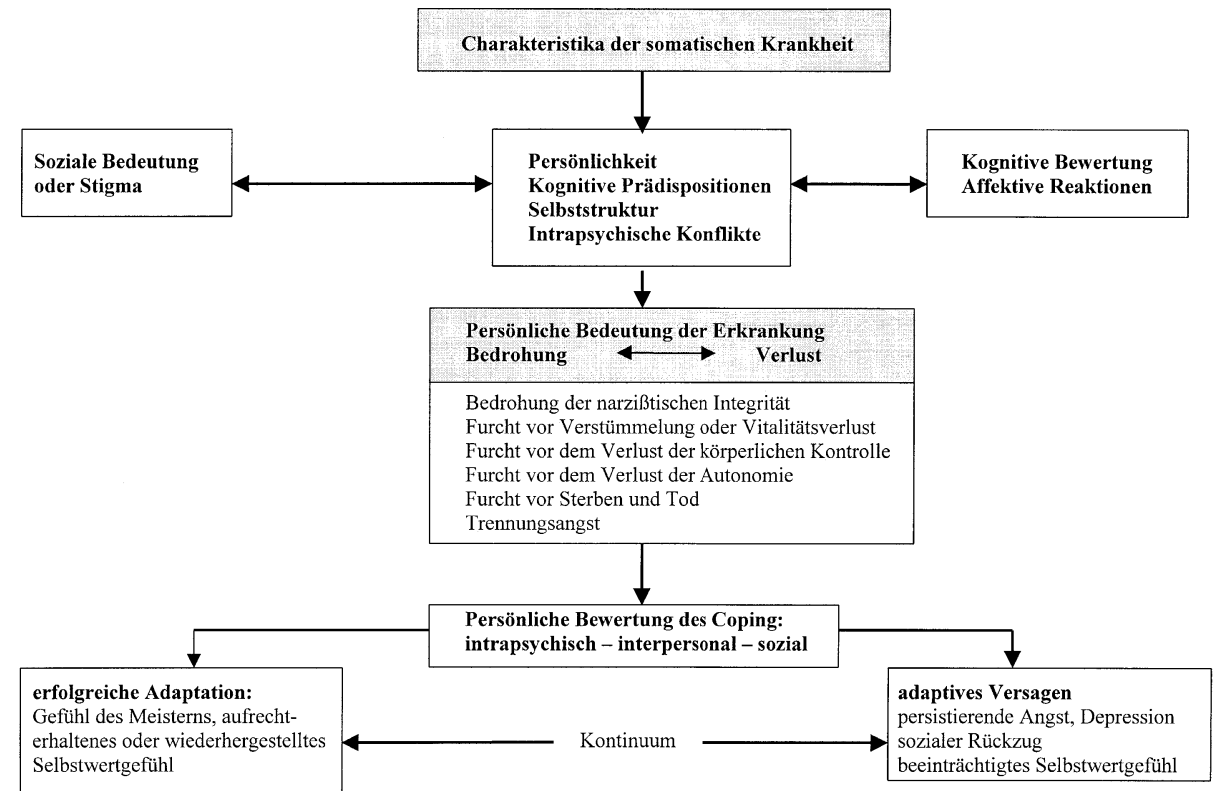


rungen an Vehemenz und drängender Schärfe zunehmen können.

Trauerarbeit. Auf der anderen Seite gewinnen Prozesse von Trauer und Depression eine eigenständige Dimension, die psychodynamisch das Gelingen oder aber auch Mißlingen der Krankheitsverarbeitung wesentlich mitbestimmen (Beutel u. Weiner 1993).

Abwehrmechanismen. Einzelne Abwehrmechanismen spielen eine bedeutsame Rolle in der Auseinandersetzung mit und der Verarbeitung der Erkrankung. So kann sich z.B. eine Verleugnung auf die Tatsache der Krankheit überhaupt, auf bestimmte Folgen der Erkrankung oder aber auf die implizite Lebensbedrohung beziehen. Verleugnung erfüllt eine adaptive Funktion beim Versuch, die übermäßige Angst und Bedrohung zu kontrollieren und enge interpersonale Beziehungen aufrechtzuerhalten. Eine maladaptive Funktion zeigt sich aber an, wenn das Ausmaß der Verleugnung eine adäquate Auseinandersetzung mit der Erkrankung grundlegend behindert und z.B. eine notwendige Trauerarbeit unmöglich macht. Aus der Perspektive der akuten und posttraumatischen Belastungsstörung können Verleugung und Intrusion als alternierende Phasen nach einer Traumaexposition mit Zuständen einer emotionalen Überkonkontrollierheit bzw. fehlenden Kontrolliertheit identifiziert werden (Horowitz 1976; s. Kap. 54).

\section{Risikofaktoren}

Kennzeichen der prämorbiden Anpassung bzw. Primärpersönlichkeit z.B. erhöhte Trait-Angst-Scores, niedriges Selbstkontrollgefühl, erlernte Hilflosigkeit und depressiogener Attributionsstil, interpersonale Dependenz, typische dysfunktionale Kognitionen usw. aber auch mangelnde soziale Unterstützung beeinflussen deutlich das Risiko einer im Kontext der somatischen Erkrankung auftretenden emotionalen Störung (Greer 1994).

Die Schwere einer Erkrankung z.B. Karzinom oder Herzinfarkt und auch spezielle medizinische Behandlungsverfahren wie chirurgische, Radio- und Chemotherapie besitzen eigenständige Bedrohungs- und Verlustkonnotationen und tragen zur psychologischen Morbidität in einer Erkrankungssituation bei (Schag $u$. Heinrich 1989).

Irreversible Folgen für das Körperbild und Selbstwerterleben aus internistischen Interventionen oder operativen Eingriffen können ebenfalls zu genuinen emotionalen Konflikten und Spannungen führen und depressive und ängstliche Reaktionen anstoßen (Harrison u. Maguire 1994).

\subsection{2}

\section{Biologische Bedingungsfaktoren}

Einzelne somatische Erkrankungen scheinen aber auch jenseits der über individuelle Bedeutungen gesteuerten psychologischen Reaktionsweisen ein differentiell ausgeprägtes Risiko hinsichtlich einer depressiven und/ oder Angststörung zu besitzen. Dies muß in Beziehung zu neurobiologischen Konzepten der Depressions- und Angstentstehung gesetzt werden. Es kann durch pathophysiologische Prozesse der Erkrankung selbst und/ oder aber auch durch interagierende Effekte von eingesetzten Medikamenten bestimmt werden.

\section{Organische Einflüsse auf depressive Symptome}

Unmittelbare organische Einflüsse können bei allen jenen Erkrankungen diskutiert werden, die entweder direkt die Integrität bestimmter neuroanatomischer Regionen oder die Funktionsweise bestimmter neurochemischer und -endokriner Schaltkreise des ZNS, die an der Regulation emotionaler Prozesse beteiligt sind, beeinträchtigen. Beispiele hierfür sind etwa die verstärkte Assoziation von depressiven Störungen bei links frontopolar oder subkortikal in den Basalganglien gelegenen Hirninfarkten (Beckson u. Cummings 1992), oder aber beim Morbus Parkinson mit Dysfunktionen nicht nur im dopaminergen, sondern auch in anderen katecholaminergen z.B. serotonergen Neurotransmittersystemen (Mayeux 1990).

Analoges gilt für zahlreiche endokrine und metabolische Erkrankungen, die entweder direkt oder indirekt zu neuroendokrinen Anomalien in der für die Entstehung depressiver Störungen als grundlegend angesehenen HPA-Achse führen. Ein Beispiel hierfür wiederum sind die häufigen Depressionen beim M. Cushing (Fava 1994).

Für eine Reihe von Medikamenten werden eigenständige depressiogene Effekte diskutiert (Patten u. Love 1997).

Von besonderem ätiopathogenetischem Interesse sind gehäufte depressive Verstimmungen bei Erkrankungen, die auf den ersten Blick weder unmittelbar das ZNS noch zentrale neuroendokrine Regulationssysteme $\mathrm{zu}$ betreffen scheinen. Ein eindrucksvolles Beispiel ist das Pankreaskarzinom, bei dem es selbst schon im zeitlichen Vorfeld der körperlichen Symptommanfestation zu zahlreichen Depressionen kommen kann (Shakin u. Holland 1988). Eine pathogenetische Hypothese bezieht sich hier auf spezielle, durch das Pankreaskarzinom produzierte Antikörper, die eine Kreuzreaktion mit zentralen Serotoninrezeptoren aufweisen (Brown u. Paraskevas 1982). Eine weitere allgemeine pathogenetische Schiene zur Auslösung von depressiven Störungen bei diversen somatischen Krankheiten könnte in zentralnervösen Interaktionen systemisch freigesetzter Zytokine bestehen (Katz 1996).

Tabelle 62.2 gibt einen Überblick über jene somatischen Krankheiten, bei denen klinisch gehäufte sym- 
Tabelle 62.2. Somatische Erkrankungen mit klarer Assoziation zu symptomatischen Depressionen. (Nach Fava u. Sonino 1996)

\begin{tabular}{|c|c|c|}
\hline Körperliche Krankheit & Klinische Charakteristika der Depression & Autoren \\
\hline \multicolumn{3}{|l|}{ Endokrine Erkrankungen } \\
\hline Morbus Cushing & $\begin{array}{l}\text { Schwere Depressionen mit Suizidrisiko möglich; sowohl bei hypophysen- } \\
\text { abhängigen als auch -unabhängigen Formen; Antidepressiva oft ineffektiv; } \\
\text { Steroidhemmer (Metyrapon, Ketoconazol) sind effektiv }\end{array}$ & Sonino et al. (1993) \\
\hline Morbus Addison & Sprechen allgemein auf Steroidsubstitution an & Fava et al. (1993) \\
\hline $\begin{array}{l}\text { Hypothyreoidismus, } \\
\text { Thyreotoxikose }\end{array}$ & $\begin{array}{l}\text { Sprechen oft auf adäquate endokrine Therapie an; manchmal Anti- } \\
\text { depressiva notwendig }\end{array}$ & Fava et al. (1993) \\
\hline $\begin{array}{l}\text { Hyperparathyreoidismus, } \\
\text { Hyperprolaktinämie }\end{array}$ & $\begin{array}{l}\text { Depression mit feindseligen Affekten assoziiert; fast nur Frauen; Bromo- } \\
\text { criptin dem Plazebo überlegen, während Amitriptylin ineffektiv }\end{array}$ & Fava et al. (1993) \\
\hline \multicolumn{3}{|l|}{ Neurologische Erkrankungen } \\
\hline Morbus Parkinson & $\begin{array}{l}\text { schwere Depression mit Suizidrisiko möglich; charakterisiert durch Angst, } \\
\text { weniger Selbstvorwurf; assoziiert mit frühem Beginn der Parkinson- } \\
\text { Erkrankung sowie bei größerer linkshemisphäraler Beteiligung; unbe- } \\
\text { handelt oft Dauer von über einem Jahr; L-Dopa ineffektiv, möglicherweise } \\
\text { depressive Symptome aggravierend, während Bromocriptin effektiv; } \\
\text { Nortriptylin dem Plazebo überlegen }\end{array}$ & $\begin{array}{l}\text { Whitlock (1982) } \\
\text { Cummings (1992) } \\
\text { Brown et al. (1988) } \\
\text { Andersen et al. (1980) }\end{array}$ \\
\hline $\begin{array}{l}\text { Zerebrovaskuläre } \\
\text { Störungen }\end{array}$ & $\begin{array}{l}\text { Major Depression mit linksfrontalen oder in Basalganglien links gelegenen } \\
\text { Läsionen, präexistenter subkortikaler Atrophie, höherem Lebensalter, } \\
\text { Heimaufenthalt, fehlender enger Beziehung assoziiert; unbehandelt oft } \\
\text { Dauer über einem Jahr; Nortriptylin dem Plazebo überlegen, Trazodon } \\
\text { hingegen nicht; elektrokonvulsive Therapie: gute Ergebnisse }\end{array}$ & $\begin{array}{l}\text { Starkstein u. } \\
\text { Robinson (1989) } \\
\text { Sharpe et al. (1994) } \\
\text { Lipsey et al. (1984) } \\
\text { Reding et al. (1986) } \\
\text { Murray u. Shea (1987) }\end{array}$ \\
\hline Chorea Huntington & $\begin{array}{l}\text { Depressive Symptome mit Wahnideen assoziiert; Response auf } \\
\text { antidepressive Medikation einschließlich MAO-Hemmer }\end{array}$ & $\begin{array}{l}\text { Folstein et al. (1983) } \\
\text { Ford (1986) }\end{array}$ \\
\hline Gehirntumoren & $\begin{array}{l}\text { Depression kann anderen Symptomen vorausgehen; oft mit anderen } \\
\text { psychischen Störungen }\end{array}$ & Lishman (1987) \\
\hline Multiple Sklerose & $\begin{array}{l}\text { Depression kann anderen Symptomen vorausgehen; variable Response } \\
\text { auf Antidepressiva: Desipramin > Plazebo, jedoch mäßige Besserung der } \\
\text { Depression; Amitriptylin > Plazebo in Kontrolle des pathologischen } \\
\text { Lachens; kognitive Therapie effektiv }\end{array}$ & $\begin{array}{l}\text { Berrios u. Quemada (1990) } \\
\text { Schiffer u. Wineman (1990) } \\
\text { Schiffer et al. (1985) } \\
\text { Larcombe u. Wilson (1984) }\end{array}$ \\
\hline Epilepsie & $\begin{array}{l}\text { Patienten unter Phenobarbital depressiver als unter Carbamazepin; } \\
\text { Amitriptylin > Plazebo }\end{array}$ & $\begin{array}{l}\text { Robertson u. Trimble } \\
(1985,1987)\end{array}$ \\
\hline Schädel-Hirn-Trauma & $\begin{array}{l}\text { Depression öfter mit rechtshirnigen Verletzungen assoziiert; Amitriptylin } \\
\text { und Phenelzin ineffektiv bei leichten gedeckten Hirnverletzungen }\end{array}$ & $\begin{array}{l}\text { Whitlock (1982) } \\
\text { Saran (1985) }\end{array}$ \\
\hline $\begin{array}{l}\text { Normaldruck- } \\
\text { hydrozephalus }\end{array}$ & Depression geht oft anderen Symptomen voraus & Whitlock (1982) \\
\hline Morbus Alzheimer & Imipramin nicht $>$ Plazebo & Reifler et al. (1989) \\
\hline Infektionserkrankungen & $\begin{array}{l}\text { Depressionen häufiger nach viralen Erkrankungen (Hepatitis, Mono- } \\
\text { nukleosis, Influenza, Enzephalitis) als nach bakteriellen Infektionen; } \\
\text { langsame Erholungsgeschwindigkeit }\end{array}$ & $\begin{array}{l}\text { Whitlock (1982) } \\
\text { Ananth et al. (1994) }\end{array}$ \\
\hline \multicolumn{3}{|c|}{ Unterschiedliche Erkrankungen } \\
\hline $\begin{array}{l}\text { Systemischer Lupus } \\
\text { erythematodes }\end{array}$ & Steroide oft erfolgreich in der Kontrolle depressiver Symptome & $\begin{array}{l}\text { Whitlock (1982) } \\
\text { Hama u. Boumpas (1995) }\end{array}$ \\
\hline $\begin{array}{l}\text { Folsäuremangel, } \\
\text { perniziöse Anämie }\end{array}$ & Sprechen allgemein auf Substitutionsbehandlung an & Eilenberg (1960) \\
\hline $\begin{array}{l}\text { Pankreas-, Lungen- } \\
\text { karzinom }\end{array}$ & Depression geht oft anderen Symptomen voraus & $\begin{array}{l}\text { Holland et al. (1986) } \\
\text { Hughes (1985) }\end{array}$ \\
\hline Aids & $\begin{array}{l}\text { Depression charakterisiert durch Lethargie und Rückzug; kann durch } \\
\text { opportunistische ZNS-Infektionen ausgelöst werden; Imipramin > Plazebo }\end{array}$ & $\begin{array}{l}\text { Perkins u. Evans (1991) } \\
\text { Judd u. Mijch (1994) } \\
\text { Rabkin et al. (1994) }\end{array}$ \\
\hline
\end{tabular}


ptomatische Depressionen angenommen werden können. Die folgende Übersicht listet Medikamente auf, die Depressionen induzieren können.

Übersicht. Medikamente, die Depressionen induzieren können. (Nach Fava u. Sonino 1996)

- Baclofen,

- Cimetidin,

- Cycloserin,

- Digitalis,

- Fenfluramin,

- Flunarizin,

- L-Dopa,

- Methyldopa,

- orale Kontrazeptiva,

- Propranolol,

- Ranitidin,

- Reserpin,

- Steroide,

- Sulfonamide.

Symptome sind in der Regel mild und sistieren nach Absetzen der Präparate, mit Ausnahme von Fenfluramin, das Depression bei Absetzen induzieren kann.

\section{Organische Einflüsse auf Angstsymptome}

Eine ganz analoge Argumentation erfolgt auch für eine verstärkte Assoziation von Angststörungen bei definierten Krankheitsbildern und bestimmten Medikamenten bzw. psychotropen Substanzen (Tabelle 62.3). Hierunter sind zunächst Erkrankungen mit direkten Effekten auf umschriebene zentralnervöse Strukturen $\mathrm{zu}$ benennen. Ein spezielles Beispiel sind Tumoren des Temporallappens bzw. psychomotorische Anfallsleiden, die gehäuft zu pathologischer Angst und Panik führen können (Strian u. Ploog 1988).

Veränderte metabolische Zustände lösen ebenso Angstsymptome aus (Lishman 1998) wie Zustände von Hypoxie z. B. bei Lungenembolie (Dietch 1981). Hyperthyreotische Krisen (Kathol et al. 1986; Trzepacz et al. 1988), Phäochromozytome (Starkman et al. 1985) sowie paraneoplastische Syndrome bei Pankreas- und Lungenkarzinomen (Holland et al. 1986) müssen in der Ätiopathogenese von Angststörungen differentiell beachtet werden.

Aus klinischer Perspektive ist es relevant, daß schätzungsweise ca. $30 \%$ der Patienten mit therapeutisch refraktären Schmerzsyndromen an unerkannten Angststörungen leiden (Bouckoms u. Hackett 1991).

\subsection{3}

\section{Soziale Bedingungsfaktoren}

Bei der Entstehung und Aufrechterhaltung von klinisch relevanten Depressionen und Ängsten stellen ein krankheitsbedingter Verlust sozialer Rollen und Akti-
Tabelle 62.3. Somatische Krankheiten und Internistika mit einer verstärkten Angstassoziation. (Nach Goldberg u. Posner 1993; Colon u. Popkin 1996)

\begin{tabular}{|c|c|}
\hline Somatische Erkrankungen & Internistika \\
\hline $\begin{array}{l}\text { Kardiovaskulär } \\
\text { Angina pectoris, } \\
\text { Arrhythmie, } \\
\text { kongestive Kardiomyopathie, } \\
\text { Hypovolämie, } \\
\text { Myokardinfarkt, } \\
\text { Herzklappenfehler }\end{array}$ & $\begin{array}{l}\text { Anästhetika/Analgetika, } \\
\text { Anticholinergika, } \\
\text { Antidepressiva (Trizyklika, } \\
\text { SSRI), } \\
\text { Antihistaminika, } \\
\text { Antihypertensiva, } \\
\text { Antibiotika, } \\
\text { Bronchodilatoren, } \\
\text { Digitalis, } \\
\text { Ethosuximid, } \\
\text { Hydralazin, } \\
\text { Insulin, } \\
\text { Kalziumkanalblocker, } \\
\text { Koffein, } \\
\text { L-Dopa, } \\
\text { Muskelrelaxantien, } \\
\text { Neuroleptika, } \\
\text { nichtsteroidale Antiphlogi- } \\
\text { stika, } \\
\text { Östrogen, } \\
\text { Procain, } \\
\text { Procarbazin, } \\
\text { Schilddrüsenhormone, } \\
\text { Schwermetalle, Toxine, } \\
\text { Sedativa, } \\
\text { Steroide, } \\
\text { Sympathikomimetika, } \\
\text { Theophyllin }\end{array}$ \\
\hline $\begin{array}{l}\text { Endokrin } \\
\text { Hyperkortisolismus, } \\
\text { Hyperkalzämie, } \\
\text { Hypokalzämie, } \\
\text { Hyperthyreoidismus, } \\
\text { Karzinoid, } \\
\text { Phäochromozytom }\end{array}$ & \\
\hline \multicolumn{2}{|l|}{ Ulcus pepticum } \\
\hline $\begin{array}{l}\text { Metabolisch } \\
\text { Hyperkaliämie, } \\
\text { Hyperthermie, } \\
\text { Hypoglykämie, } \\
\text { Hyponatriämie, } \\
\text { Hypoxie, } \\
\text { Porphyrie }\end{array}$ & \\
\hline $\begin{array}{l}\text { Neurologisch } \\
\text { Akathisie, } \\
\text { Enzephalopathie, } \\
\text { Massenblutung, } \\
\text { postkontussionelles Syndrom, } \\
\text { epileptische Anfälle, } \\
\text { organischer Schwindel }\end{array}$ & \\
\hline $\begin{array}{l}\text { Respiratorisch } \\
\text { COPD, } \\
\text { Pneumothorax, } \\
\text { Lungenödem, } \\
\text { Lungenembolie }\end{array}$ & \\
\hline $\begin{array}{l}\text { Immunologisch } \\
\text { Anaphylaxie, } \\
\text { systemischer Lupus erythema }\end{array}$ & \\
\hline
\end{tabular}


vitäten, eine verstärkte soziale Isolierung und Entfremdung, eine Stigmatisierung durch die Erkrankung sowie finanzielle Probleme infolge der Erkrankung eigenständige Bedingungsgrößen dar (Rodin et al. 1991). Umgekehrt können wichtige soziale Unterstützungen in partnerschaftlichen und familiären Beziehungen sowie positive Einflüsse eines tragenden sozialen Netzes den negativen emotionalen Folgen einer somatischen Erkrankung bedeutsam moderierend entgegenwirken (Dew et al. 1990). Bei chronischer Progredienz einer Erkrankung und zunehmender Konfrontation mit dem Sterben treten möglicherweise negative soziale Bedingungsfaktoren eher in den Hintergrund. Die Bedeutung vorhandener oder aber fehlender interpersonaler und sozialer Unterstützungen nimmt hingegen noch zu. Bei einer chronischen Persistenz von krankheitsbedingten Symptomen und psychosozialen Folgen müssen aber auch die kurz skizzierten sozialen Rahmenbedingungen stets mitbedacht werden.

Wenn auch biologische Faktoren für die Akutmanifestation von emotionalen Störungen bei einer somatischen Krankheit wie z.B. hirnlokalisatorische Aspekte bei Schlaganfällen grundlegend sein mögen, so können diese organischen Faktoren im weiteren Verlauf oft nicht mehr als entscheidend diskriminierende Variablen nachgewiesen werden. Für die Remissionswahrscheinlichkeit bzw. das Risiko einer Chronizität der psychischen Störungen nach einem Hirninsult scheinen soziale Einflüsse von größerer Wichtigkeit zu sein (Robinson 1998).

\subsection{4}

Bedingungsgefüge am Beispiel „Krebserkrankung"

Als ausgewähltes Beispiel zur Illustration des komplexen Bedingungsgefüges von somatischer Krankheit und psychischer Störung wurde an dieser Stelle Depression und Suizidalität bei Krebserkrankungen gewählt (Abb. 62.2; Kapfhammer1996):

\section{Depressivität, affektive Störung und Risiko für eine spätere Tumorerkrankung}

Epidemiologische Langzeitstudien belegen mehrheitlich, daß depressive Dispositionen oder Persönlichkeitsmerkmale bei körperlich gesunden Personen aus der Allgemeinbevölkerung keinen oder einen nur geringen linearen Vorhersagewert für eine Krebserkrankung nach 10-20 Jahren besitzen. In kontrollierten Untersuchungen an Patienten mit affektiven Störungen läßt sich zwar ein erhöhtes Risiko für eine Reihe von kardiovaskulären, pulmonalen, atopischen und metabolischen Krankheiten, nicht aber für Karzinome belegen (Kapfhammer 1993a).

\section{Negative Lebenseregnisse, Depressivität und Tumorauslösung}

Die moderne Life-event-Forschung verneint einen unmittelbaren Zusammenhang zwischen gehäuften nega-

Abb. 62.2. Zusammenhang von Depression und Tumorerkrankung. (Nach Kapfhammer 1993 a)

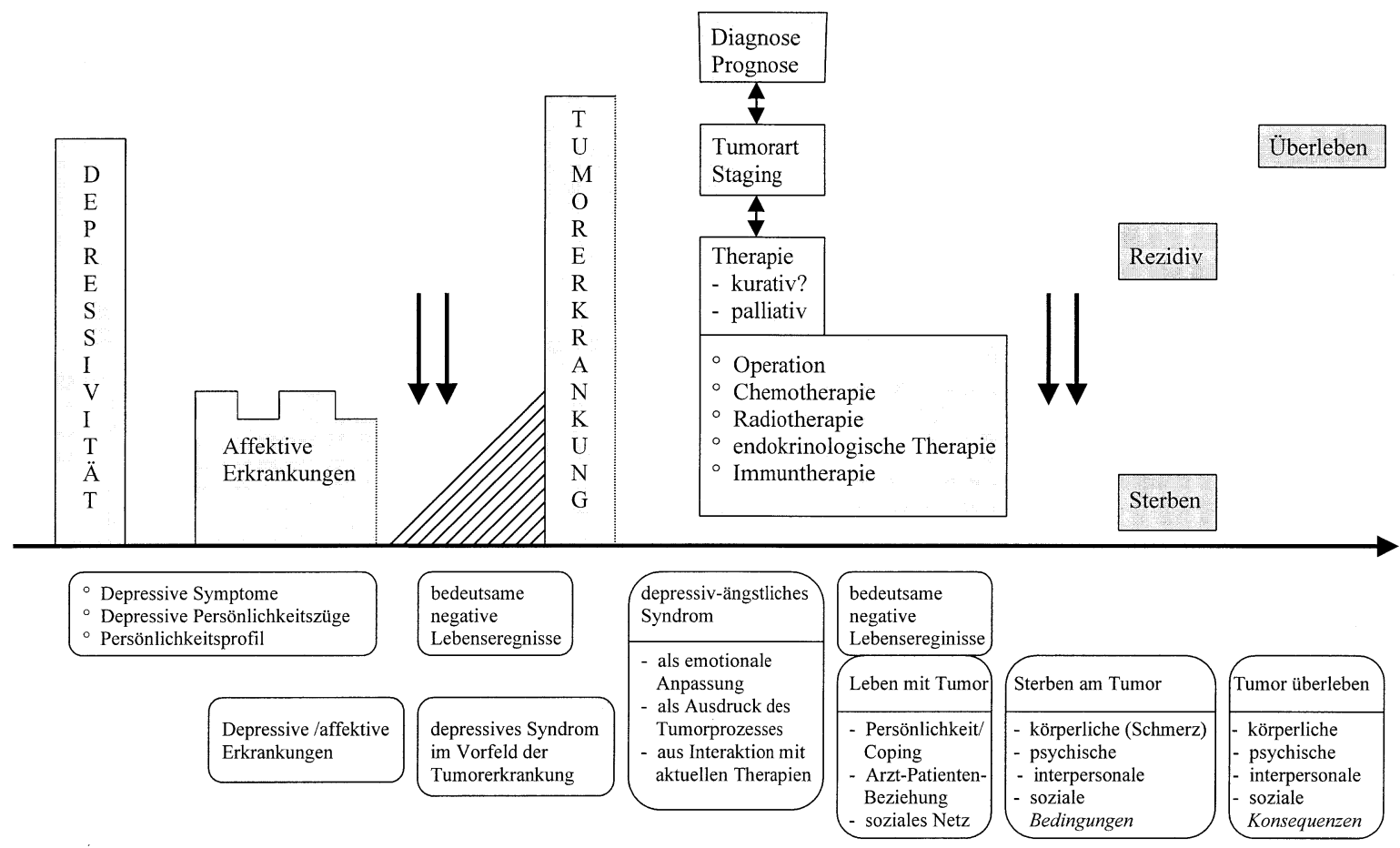


tiven Lebensereignissen, v. a. Verlusterlebnissen, einer hierdurch angestoßenen Depressivität und einer verstärkten Auftretenswahrscheinlichkeit von Tumorerkrankungen. Wenngleich Zusammenhänge zwischen Verlusten, depressiver Trauer und veränderten Immunreaktionen nachgewiesen werden können, so ist die Bedeutung für die Auslösung einer Tumorerkrankung weiterhin unklar (Levenson u. Bemis 1991).

\section{Prävalenz von depressiven Störungen bei Tumorpatienten}

Angaben zur Häufigkeit von depressiven Syndromen sind in einem hohen Maße abhängig von der Untersuchungsmethodologie. Stützt man sich in der Bewertung lediglich auf Studien mit einer strukturierten Erfassung depressiver Störungen, so liegen die Raten für eine Major Depression (DSM-III/IV) zwischen 6 und ca. $20 \%$. Bei einer ca. 6\%igen Sechsmonatsprävalenz für eine Major Depression in der Allgemeinbevölkerung ist es realistisch, die Erkrankungshäufigkeit bei Tumorpatienten auf 2- bis 4 fach erhöht zu schätzen. Sie bewegt sich somit in einer vergleichbaren Größenordnung wie bei anderen schweren somatischen Erkrankungen. Hierbei ist aber zu beachten, daß mit der Schwere des Tumorleidens, aber auch mit der Progression in ein Terminalstadium die Prävalenzzahlen noch deutlich ansteigen (Massie u. Holland 1990).

Berücksichtigt man zusätzlich die Häufigkeiten von Anpassungsstörungen mit depressiver/ängstlicher Stimmung, Angst- und organisch bedingten psychischen Störungen, so ist klinisch festzuhalten, daß bei ca. der Hälfte der Krebspatienten im Laufe einer Tumorerkrankung psychiatrisch und/oder psychotherapeutisch behandlungsbedürftige seelische Störungen vorliegen (Abb. 62.3; Derogatis et al. 1983).

\section{Schwierigkeiten in der Diagnosestellung depressiver Störungen}

Die Eröffnung der Diagnose „Krebs“ stellt trotz bedeutsamer therapeutischer Fortschritte nach wie vor eine existentielle Bedrohung für die meisten Betroffenen dar. Zentrale Ängste können sich auf so unterschiedliche Aspekte wie Sterben und Tod, übermäßige Abhängigkeit von familiärer Betreuung und ärztlicher Versorgung, entstellende Operationen mit Beeinträchtigungen im Selbstwerterleben und Körperbild, Verlust des beruflichen Status und soziale Isolation, Labilisierung persönlicher Bande, körperliches Unwohlsein und Schmerz beziehen. Je nach Persönlichkeit, psychosozialer Fertigkeit und soziokultureller Verankerung nimmt die emotionale Reaktion eines Krebspatienten eine unterschiedliche Gestalt an und variiert das Gelingen in der vielschichtigen Auseinandersetzung mit der Erkrankung.

Ärzte und Pflegepersonen verkennen häufig das Vorliegen einer behandlungsbedürftigen und auch behandelbaren Depression. Zum einen begnügen sie sich, in

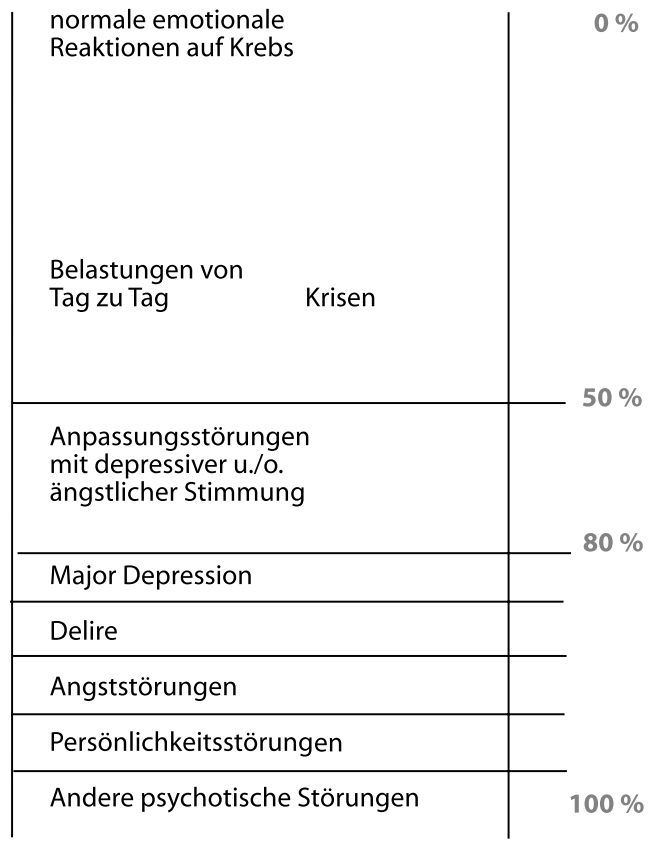

Abb. 62.3. Spektrum psychischer Störungen bei Tumorerkrankungen. (Nach Derogatis et al. 1983)

ihr lediglich eine ,angemessene“ emotionale Reaktionsweise angesichts einer oft tödlichen Bedrohung zu erblicken. Zum anderen herrscht nicht selten ein therapeutischer Nihilismus gegenüber der psychotherapeutischen und/oder psychopharmakologischen Beeinflußbarkeit dieser Affektstörungen und Verstimmungen vor.

Im Diagnoseprozeß selbst können sich zusätzliche Probleme ergeben, wenn v. a. somatische Symptome wie z. B. Müdigkeit, Energieverlust, Antriebsschwäche oder Abnahme des Körpergewichts sowohl integraler Bestandteil einer depressiven Verstimmung als auch Ausdruck der Tumorerkrankung bzw. aktueller Therapien sein kann.

Entscheidendere Hinweise auf das Vorliegen einer depressiven Störung ergeben sich durch die sorgfältige Exploration kognitiver und affektiver Symptome wie z. B. Schuld-, Versagensgefühle, Interesseverlust, Bestrafungsüberzeugung, Entscheidungsambivalenz, Selbstwertverlust, Hoffnungslosigkeit, Suizidgedanken. Für ein sicheres Erkennen von depressiven Störungen bei Krebspatienten ist also neben der psychodynamischen Erlebnisdimension auch die psychopathologische Symptomebene von grundlegender Bedeutung.

\section{Persönlichkeitstypus, Depressivität und Tumorprogression}

Dem klinisch tätigen Arzt können einige Indikatoren an die Hand gegeben werden, die es ihm erleichtern zu erkennen, welche Patienten mit einem erhöhten Risiko im 
Laufe einer Tumorerkrankung auch psychisch dekompensieren können. Sie helfen ihm, bei diesen Patienten besonders sorgfältig nach depressiven Symptomen zu fragen. Unter den Kennzeichen sind v. a. eine psychiatrische Anamnese, ein labiles Selbstwerterleben, mangelhafte soziale Unterstützung, behandlungsbezogene Komplikationen oder Toxizitätszeichen (s. unten), der Schweregrad der körperlichen Symptome sowie persistierend ungelöste Besorgnisse z.B. im Körperbilderleben zu nennen (Harrison u. Maguire 1994).

Ein Persönlichkeitstypus wird gehäuft unter Krebspatienten angetroffen. Er zeichnet sich durch eine hohe passive Kooperativität im Behandlungsverlauf, eine auffällige Orientiertheit an äußerlichen Normen, eine geringe Selbstbehauptung, v. a. aber eine ausgeprägte Tendenz zur Unterdrückung zentraler Gefühlsregungen, speziell von aggressiven Affekten aus. Ein gelegentlich gefundener Zusammenhang dieses Typus $\mathrm{C}$ mit einer schnelleren Tumorprogression muß nach neueren Ergebnissen aus prospektiven Studien wieder offen gehalten werden. Da mit dem Typus $\mathrm{C}$ aber wichtige andere Persönlichkeitscharakteristika wie innere Kontrollüberzeugung und Copingstil einhergehen, sind Auswirkungen auf die Verarbeitung des Krankheitsgeschehens und die Lebensqualität als sehr wahrscheinlich anzunehmen (Spiegel 1991).

\section{Tumorart und Depressivität}

Einzelne Tumorarten lassen möglicherweise eine differentielle Assoziation mit depressiven Störungen erkennen.

Beispiel ist das Pankreaskarzinom, das bei ca. 50\% der betroffenen Patienten bereits Monate vor diagnostizierbaren somatischen Beschwerden mit klinisch relevanten depressiven Verstimmungen einhergeht.

Primäre Gehirntumoren, v. a. bei einer linkstemporalen Lokalisierung, Tumoren aus Organen mit starker zerebraler Metastasierung wie Hypernephrom, Lungenund Mammakarzinom sowie hormonproduzierende Tumoren der Hypophyse und Nebennierenrinde zeigen ebenfalls ein gehäuftes Auftreten von depressiven Störungen.

Als wichtige biologische Vermittlungsmechanismen werden u. a. tumorproduzierte Antigene mit Interaktionen an Neurotransmitterrezeptoren, ektope ACTH-Sekretion, sekundärer Hyperkortisolismus, Hyperkalzämien sowie eine Produktion von psychoaktiven Substanzen diskutiert (Rodin et al. 1991).

Während bei diesen Tumorarten vorrangig biologisch-inhärente Faktoren in der Pathogenese zu beachten sind, wirken andere Tumorarten wie z. B. Karzinome im Mund- und Rachenbereich, aber auch Kolon- und Genitalkarzinome, die häufig entstellende Operationen nach sich ziehen, in erster Linie durch negative Interferenzen mit unterschiedlichen psychosozialen Bereichen depressionsfördernd (Liang et al. 1990).

\section{Stadium der Tumorerkrankung und Depressivität}

Zur Beziehung von Depressivität und Krankheitsstadium lassen sich folgende Aspekte herausstellen: Die Schwere der Erkrankung, wie sie z. B. über den Karnofsky-Index gemessen werden kann, chronische Schmerzsyndrome, tumorbedingte Anorexie, Übelkeit und Erbrechen tragen signifikant zu einer Depressivität bei. Als Einzelvariable scheint Depressivität einen nur geringen Einfluß auf die quantitative Überlebenszeit auszuüben, wohl aber auf die Qualität in der individuell jeweils verbleibenden Lebensspanne.

Wenngleich depressiv-ängstliche Verstimmungen im ersten Jahr nach einer Diagnosestellung in der Regel signifikant abnehmen, leidet ca. ein Drittel der Patienten auch nach dieser Zeit noch an psychiatrisch und psychotherapeutisch behandlungsbedürftigen Beschwerden. Selbst bei einer somatisch überwundenen Tumorerkrankung müssen die erlebnisreaktiven Konsequenzen, die Auswirkungen auf Partnerschaften, Sozialkontakte und berufliche Leistungsfähigkeit als mögliche Bedingungsfaktoren für depressive Störungen bedacht werden (Kapfhammer 1993 a).

\section{Onkologische Therapiemodalitäten und Depressivität}

Unter einigen chemotherapeutischen Behandlungsregimen treten wahrscheinlich gehäuft depressive Störungen auf. Erhöhte depressiogene Effekte werden für die Einzelsubstanzen Vincristin, Vinblastin, L-Asparaginase, Decarbazin, Procarbazin, Hexamethylamid sowie für die Kombination von Kortison, Medroxyprogesteronazetat und Aminogluthetimid diskutiert (Cull 1990). Eigenständige depressiogene Effekte müssen ferner bei einer Behandlung mit Glukokortikoiden, Interferon und einer Radiatio des Gehirns berücksichtigt werden (Lesko et al. 1993).

Psychopathologisch bedeutsam ist, daß die ausgelöste depressive Verstimmung häufig mit Einbußen in kognitiven Funktionen oder mit Symptomen eines Delirs einhergehen kann. Neben diesen toxischen Effekten spielen aber auch andere assoziierte Variablen wie induzierte Übelkeit, Erbrechen und somatische Dysfunktionen, emotionale Reaktionen auf ein verändertes Körperbilderleben sowie Besonderheiten des psychosozialen Umfelds eine wichtige Rolle in der Auslösung pharmakogener depressiver Verstimmungen.

\section{Suizidalität und Tumorerkrankung}

In einer vertrauensvollen und sicheren Beziehung zum Arzt gelingt es einem Krebspatienten, auch offen über vorhandene suizidale Gedanken und Phantasien zu sprechen. Klinisch scheint diese mitgeteilte Suizidalität häufiger vorzukommen, als dies in nüchternen Forschungsinterviews eruiert werden kann. Vor allem die psychodynamische Bedeutung für die subjektive Gefühlskontrolle und die Aufrechterhaltung von Autonomie des betroffenen Patienten ist hierbei zu beachten, 
ohne daß damit bereits eine ernsthafte Selbstgefährdung einhergehen muß. In der ärztlichen Führung von Krebspatienten können folgende Faktoren hilfreich zur Erkennung des Suizidrisikos sein:

- Krankheitsprogression,

- schlechte Prognose,

- unkontrollierte Schmerzsymptomatik,

- Depression und Hoffnungslosigkeit,

- Delir und Enthemmung,

- Kontrollverlust,

- vorbestehende Psychopathologie,

- frühere Suizidanamnese,

- Suizid in der Familie,

- umfassende Erschöpfung.

Das Suizidrisiko von Krebspatienten ist im Vergleich zur Allgemeinbevölkerung erhöht, bei Männern etwas höher als bei Frauen. Es betrifft alle Altersgruppen, v. a. aber über 50jährige und besonders auch jüngere Männer. Es ist im ersten Jahr nach Diagnosestellung am höchsten, nimmt dann signifikant wieder ab und gleicht sich nach wenigen Jahren dem allgemeinen Suizidrisiko an. Patienten ohne Behandlung, aber auch solche mit verstümmelnden operativen Eingriffen tragen ein erhöhtes Risiko, ebenso Patienten, die nach vorausgegangener Remission überraschend ein Rezidiv erleiden. Von klinisch großer Bedeutsamkeit ist, daß mit der Progression einer Krebserkrankung zwar die Depressivität und die Ängstlichkeit an Häufigkeit und Intensität zunehmen, das Suizidrisiko aber nicht analog erhöht ist (Wolfersdorf 1993).

\section{4}

\section{Symptomatologie}

\section{Depressive Störungen}

Es herrscht nach wie vor ein inkonsistentes Meinungsbild in der Literatur darüber vor, ob depressive Störungen, die in einem engen zeitlichen und auch kausalen Kontext einer somatischen Krankheit auftreten, ein charakteristisches symptomatologisches Bild zeigen. Nur wenige Studien widmeten sich bisher diesem Sachverhalt.

Clayton u. Lewis (1981) hoben in ihrer Studie zunächst eine phänomenologische Ähnlichkeit von sekundären Depressionen bei somatischen Erkrankungen und primären affektiven Störungen hervor. Sie fanden bei der ersten Gruppierung aber ein Überwiegen von Männern, einen früheren Beginn, eine erhöhte familiäre Prävalenz für Alkoholismus und eine normale REM-Latenz.

Freedland et al. (1992) studierten depressive Patienten mit koronaren Herzerkrankungen. Eine Subgruppe mit einer positiven Anamnese für frühere Depressionen war häufiger weiblich und jünger, zeigte einen höheren Score im Beck Depressionsinventar. Bei ihnen imponierten symptomatologisch häufiger Versagensgefühle, Selbstvorwürfe, Hoffnungslosigkeit und Weinen. Die andere Subgruppe ohne frühere Depressionsanamnese war häufiger männlich und älter.

Winokur (1990), ein Verfechter des diagnostischen Konzeptes der „sekundären Depression“ bei somatischen Krankheiten, unterstrich gegenüber einer primären Major Depression folgende diskriminierenden Kennzeichen:

- höheres Alter bei Beginn,

- größere Wahrscheinlichkeit einer positiven Response auf Elektrokrampftherapie,

- größere Wahrscheinlichkeit eines gebesserten Status bei Entlassung,

- häufigere assoziierte „organische“ Zeichen im psychopathologischen Status,

- niedrigere familiäre Belastungen für Alkoholismus und Depression (19\% bei somatischen Patienten vs. $36 \%$ bei psychiatrischen Patienten),

- seltenere Suizidgedanken und vollzogene Suizide (10\% Mortalität durch Suizid bei somatischen Patienten vs. $45 \%$ bei psychiatrischen Patienten).

\section{Symptome als Diskriminatoren}

Auch Moffic u. Paykel (1975) betonten in ihrer Untersuchung das geringere Ausmaß an Suizidalität bei depressiven Patienten mit somatischen Krankheiten. Sowohl ihre Studie als auch die Studie von Clark et al. (1983) setzte als diagnostisches Instrument das Beck Depressionsinventar ein. Letzere Untersuchung führte 2 Vergleiche durch:

- psychiatrische Patientengruppe mit Major Depression $(\mathrm{n}=101)$ vs. gesunde Kontrollgruppe und

- somatisch kranke Patientengruppe mit und ohne Depression $(\mathrm{n}=335)$.

In beiden Vergleichsgruppierungen bildeten die BDI-Scores eine zugrunde liegende Dimension der Depressionsschwere ab. 14 Symptome erwiesen sich im ersten Vergleich als signifikante Diskriminatoren, 7 Symptome im zweiten Vergleich, von denen 6 auch im ersten Vergleich gut diskriminierten (Tabelle 62.4). Diese 6 Symptome (Suizidgedanken, Versagensgefühl, Bestrafungsgefühl, Verlust an sozialem Interesse, Entschlußunfähigkeit und Unzufriedenheit) schienen also durch die somatische Erkrankung und den hiermit assoziierten Distreß nicht konfundiert zu sein.

Von klinischem Interesse war, daß das Symptom „Weinen“ bei der medizinisch kranken Patientengruppe als Diskriminator imponierte. Das Vorliegen dieses Symptoms rechtfertigt somit, einen Patient intensiver nach einer koexistenten Depression zu befragen.

Wiederum zeigten 8 Symptome (Hoffnungslosigkeit, Schuld, Selbsthaß, Selbstvorwurf, Irritabilität, schlech- 
Tabelle 62.4. Symptome (Beck Depressionsinventar), die signifikant zwischen depressiven und nichtdepressiven medizinisch kranken und psychiatrisch kranken Patienten diskriminieren. (Nach Creed 1997)

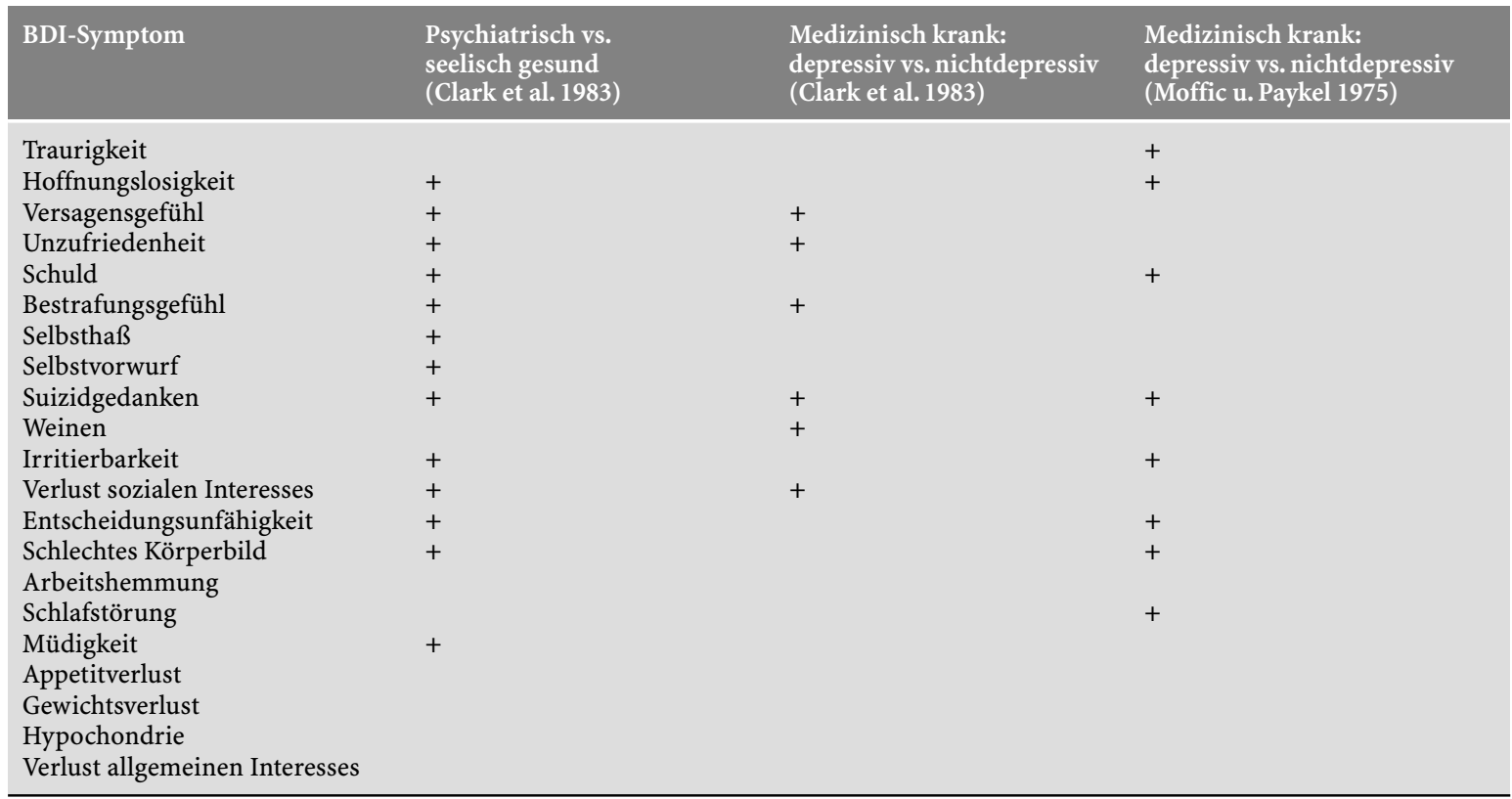

tes Körperbild, Arbeitshemmung, und Müdigkeit), die noch klar im ersten Vergleich zwischen psychiatrischen Patienten mit einer Major Depression und gesunden Kontrollprobanden unterschieden, in der somatisch kranken Patientengruppe ihre diskriminative Potenz nicht mehr. Diese depressiven Symptome schienen also bedeutsam durch die somatische Krankheit selbst bzw. die Reaktion hierauf, möglicherweise aber auch durch das höhere Lebensalter ( 57 vs. 39 Jahre) betroffen zu sein.

Die im BDI integrierten somatischen bzw. psychovegetativen Symptome erwiesen sich interessanterweise in beiden Vergleichsgruppierungen als wenig diskriminativ. Hier bestand eine gewisse Diskrepanz zur Studie von Moffic u. Pakel (1975), die zwar ebenfalls das häufige allgemeine Vorkommen dieser Symptome bei medizinischen Patienten feststellten, einigen Symptomen aber eine gewisse Unterscheidungskraft zusprachen. In einer näheren Betrachtung des Schweregrads einer depressiven Verstimmung zeichnete sich bei der psychiatrischen Subgruppe ab einer bestimmten Intensitätsschwelle eine Koexistenz quasi aller 14 diskriminativen Symptome ab, während die 7 diskriminativen Symptome bei der medizinischen Subgruppe eher kumulativ zur Depressionsschwere beitrugen.

In einer weiteren Studie zeigten Clark et al. (1998), daß die symptomatologische Kombination aus Freudlosigkeit (Anhedonie), niedrigem positivem Affekt (mangelnde lustvolle Auseinandersetzung mit der Umwelt, Desinteresse, niedrige Motivation, sozialer Rückzug) und hohem physiologischem Arousal am besten depressive Patienten mit somatischen Krankheiten gegenüber depressiven psychiatrischen Patienten und gesunden Kontrollprobanden unterscheiden konnte.

\section{Angststörungen}

Das symptomatologische Bild von Angststörungen im Kontext somatischer Krankheiten, sei es infolge akuter angstvoller Reaktionen auf die Erkrankung und die erwarteten Behandlungsprozeduren, sei es infolge der Exazerbation vorbestehender Angststörungen oder der Auslösung von Angststörungen als unmittelbarer Ausdruck des Krankheitsgeschehens oder intervenierender Therapien, unterscheidet sich zunächst sehr wenig von Angststörungen ohne diese Rahmenbedingungen. Dies trifft auf die Charakteristik akuter Angst- und Paniksymptome, phobischen Erlebens und Vermeidungsverhaltens, generalisierter Angstzustände und posttraumatischer Streßsymptome zu (Noyes u. Hoehn-Saric 1998).

Angstinhalte. Hoch bedeutsam müssen aber die typischen Angstinhalte aus der Konfrontation mit bestimmten somatischen Erkrankungen und bevorstehenden Therapiemaßnahmen angesehen werden (s. oben). Typisch sind wiederkehrende intrusive Gedanken und Vorstellungen von Krankheit und Unfall, von körperlicher Verletzung und Tod bei Aufnahme in ein Krankenhaus (Kaasa et al. 1993; Stefanek et al. 1989). Typisch sind ferner Ängste vor der Wiederkehr eines erlittenen somatischen Ereignisses z. B. eines Myokardinfarkts oder Apoplexes, eine leicht generalisierende antizipatorische Angst vor Umständen und Aktivitäten, die als gefährlich für ein solches Rezidiv erachtet wer- 
den. Diese Ängste erschweren das Verlassen der mit Sicherheit und Schutz verknüpften Station im Krankenhaus, den Wechsel in eine rehabilitative Einrichtung, die Wiederaufnahme der gewohnten körperlichen und sozialen Aktivitäten im Alltagsleben (Bishay et al. 1995; Burvill et al. 1995; Maguire u. Haddad 1996).

Angst nach intensivmedizinischer Behandlung. Besondere Probleme erwachsen bei jenen Patienten, die infolge eines allgemeinen Kreislaufversagens oder einer schweren Lungenerkrankung über längere Zeit einer respiratorischen Unterstützung bedurften, vom Respirator aufgrund der allgemeinen Statusbesserung entwöhnt werden könnten, aber nunmehr mit heftiger Angst und Panik reagieren (De Vito 1990). Die in der Entwöhnungsphase typischerweise auftretende Dyspnoe kann über den biologischen Mechanismus eines „falschen Erstickungsalarms“ (Klein 1993) geradezu einen Panikzustand triggern. Längerfristig anhaltender respiratorischer Disstreß bei intensiv medizinisch behandelten Patienten nach akutem Lungenversagen (ARDS) muß als wichtige Bedingung für das Auftreten von posttraumatischen Belastungsstörungen mit häufig chronischer Verlaufstendenz identifiziert werden (Kapfhammer et al. 2001).

\section{5}

\section{Verlauf und Prognose}

\section{Depressivität}

Sorgfältige Nachuntersuchungen belegen, daß die in ei- nem stationären Rahmen erkennbaren depressiven Verstimmungen nicht einfach nur vorübergehender Natur sind, sondern in $30-50 \%$ der Fälle auch noch nach einem Jahr als behandlungsbedürftig eingestuft werden müssen (Mayou et al. 1988).

Hiermit geht eine intensivere Beanspruchung diverser ambulanter medizinischer und sozialer Einrichtungen einher (Cassem 1995; Katon 1998). Die adäquate Behandlung z.B. von depressiven Patienten, die ein intensives medizinisches Inanspruchnahmeverhalten zeigten („high-utilizers“), konnte hingegen signifikant die Anzahl der Arbeitsunfähigkeitstage und die Scores auf diversen Beeinträchtigungsskalen bei einem EinjahresFollow-up reduzieren (von Korff et al. 1992).

\section{Angststörung}

Im Zuge der somatischen Erkrankung neu aufgetretene Angststörungen, diagnostisch zumeist Anpassungsstörungen, zeigen überwiegend eine günstige Verlaufstendenz. Der angstvolle Zustand bessert sich in aller Regel bei Stabilisierung des medizinischen Status auch ohne spezifische Interventionen, wobei Unterstützung und Versicherung jedoch in der Führung des Patienten bedeutsam sind (Kathol u. Wenzel 1992). Popkin et al.
(1990) fanden in ihrer Studie an konsiliarisch untersuchten Patienten mit einer Anpassungsstörung eine zufriedenstellende symptomatische Remission in 2 Drittel nach 30 Tagen. Maguire et al. (1978) registrierten in ihrer Längsschnittsstudie an Patientinnen mit Mammakarzinom und bedeutsamen Angstsymptomen vor der Mastektomie eine sukzessive Abnahme der pathologischen Angst im weiteren Verlauf. Bei $12 \%$ der Patientinnen dauerte die Angststörung zwischen 2 und 8 Monaten, $17 \%$ hatten mehr als 8 Monate Angst und bei $4 \%$ bestanden auch noch nach einem Jahr behandlungsbedürftige Angstsymptome.

Angststörungen, die in einen engen zeitlichen und kausalen Konnex eines medizinischen Krankheitsfaktors zu stellen sind, remittieren in aller Regel bei internistischer Korrektur z. B. einer Hyperthyreose vollständig und erfordern keine zusätzliche weiterführende psychiatrische Behandlung (Kathol et al. 1986). Umgekehrt gilt dies nicht unbedingt in jedem Fall auch für typische symptomatische Depressionen z. B. bei Hypothyreose, bei der auch nach internistisch ausgeglichener Schilddrüsenhormonsituation das affektive Syndrom fortbestehen kann und dann eine gezielte antidepressive Medikation erfordert (Fava 1994).

Bei schweren Angststörungen, speziell bei posttraumatischen Belastungsstörungen sollte nicht automatisch von einer hohen Spontanremission ausgegangen werden (s. oben). Dies gilt besonders für jene Patienten, die bereits vor ihrer somatischen Krankheit an bedeutsamen Angststörungen gelitten haben und bei denen die Erkrankung geradezu zu einer Bestätigung katastrophisierender Kognitionen und pathologischer Besorgnisse geführt hat. Spezielle Beachtung müssen auch solche Patienten erfahren, bei denen es infolge der gewählten Therapieform jederzeit zu einer Aktualisierung der Angst- und Panikerfahrung kommen kann, z. B. bei Patienten mit implantierten Kardioversionsdefibrillatoren (Pauli et al. 1999).

\section{Verlauf}

In der Verlaufsperspektive müssen nicht nur eine erhöhte assoziierte soziale Behinderung bei koexistenten depressiven und Angststörungen (Sherbourne et al. 1996; Wells et al. 1989), sondern auch eine insgesamt erhöhte Morbidität und Mortalität diskutiert werden (FrasureSmith et al. 1993; Hayward et al. 1990; Morris et al. 1993). Während die erhöhte Morbidität möglicherweise durch eine Symptomamplifikation, eine verstärkte Tendenz zu Somatisierung und hypochondrischer Fixierung mit erklärt werden kann (Katon 1998), müssen für die erhöhte Mortalität vielfältige Mechanismen diskutiert werden, z. B. suizidales und parasuizidales Verhalten, eine durch die depressive Verstimmung reduzierte Compliance bezüglich lebensnotwendiger Medikamente, pathophysiologisch durch depressive oder Angstaffekte vermittelte negative Effekte auf den Krankheits- 
prozeß selbst z. B. vermehrte kardiale Arrhythmien usw. (Bigger et al. 1992; Katon u. Sullivan 1990; Klein et al. 1995).

\section{6}

\section{Diagnostik und Differentialdiagnose}

Die in Abschn. 62.1 aufgeführten diagnostischen Kategorien der beiden aktuellen psychiatrischen Klassifikationssysteme von ICD-10 und DSM-IV stehen prinzipiell zur Verfügung, die im psychiatrischen Konsiliardienst vorgestellten depressiv-ängstlichen Störungen diagnostisch zu erfassen. Die zahlreichen vorliegenden Kategorien sind aber nicht unproblematisch, da sie speziell für den Manifestationskontext somatischer Erkrankungen meist nicht oder nur sehr unzureichend validiert worden sind. Dies betrifft speziell die diagnostische Wertigkeit von somatischen und psychovegetativen Symptomen, aber auch den Schweregrad von emotionalen Störungen.

Zur Abschätzung der diagnostischen Wertigkeit wurden unterschiedlichste Strategien empfohlen. Für alle Strategien lassen sich in Abhängigkeit von dem empirischen Forschungsziel einerseits, von der Nützlichkeit im klinischen Alltag andererseits wichtige Argumente aufführen, mit jeweils unterschiedlichen Konsequenzen für Reliabilität, Sensitivität und Spezifität der erhobenen Daten (Tabelle 62.5). Wenige empirische Studien haben sich bisher diesem Thema gewidmet (Chochinov et al. 1994; Kathol et al. 1990; Koenig et al. 1995, 1997).

Schwierigkeiten beim Abschätzen des Schweregrads der emotionalen Störung entstehen durch das häufige
Vorkommen von unterschwelligen depressiven und Angststörungen im klinischen Kontext, die nicht immer adäquat durch die Kategorie der Anpassungsstörung erfaßt werden (von Ammon Cavenough 1995).

\section{Pragmatische diagnostische Haltung}

Für eine pragmatische diagnostische Haltung in der konsiliarpsychiatrischen Tätigkeit empfiehlt es sich, von den großen Kategorien der Major Depression, Panikstörung, generalisierten Angststörung, spezifischen Phobien und posttraumatischen Belastungsstörung auszugehen. Der Tatsache häufiger subschwelliger emotionaler Störungen im Konsiliarbereich begegnet man am ehesten, wenn man von einem Spektrum unterschiedlichster Schweregrade ausgeht und hierauf die Anzahl der erhebbbaren Einzelsymptome bezieht.

Vorliegende somatische Symptome sollten hierbei gleichwertig gezählt werden, wenn sie für den betroffenen Patienten eine subjektiv deutliche Intensität angenommen haben, unverhältnismäßig schwer angesichts des vorliegenden somatischen somatischen Status erscheinen und/oder in einer engen zeitlichen Korrelation $\mathrm{zu}$ anderen kognitiven und affektiven Symptomen stehen (von Ammon Cavenough 1984, 1995).

Die diagnostischen Kategorien ,infolge eines medizinischen Krankheitsfaktors“ bzw. „infolge einer Substanzinduktion" sollten nur vorsichtig und bei überzeugender Nachweismöglichkeit verwendet werden. Eine Orientierung an empirisch gesicherten Assoziationen zu einzelnen somatischen Krankheiten bzw. pharmakologischen Substanzen ist vorteilhaft (s. Abschn. 62.3.2). Diagnostisch überzeugender ist es aber, spezifische Krankheitsfaktoren und medikamentöse Therapien eigenständig auf Achse III zu führen.

Tabelle 62.5. Ansätze zur Diagnose von depressiven Störungen bei körperlich kranken Patienten. (Nach Cohen-Cole u. Stoudemire 1987)

\begin{tabular}{|c|c|c|c|c|}
\hline Ansatz & Kernmerkmale & Stärken & Schwächen & Kommentar \\
\hline Inklusiv & $\begin{array}{l}\text { Zählen aller möglichen } \\
\text { Symptome, unabhängig ob } \\
\text { sie durch die zugrunde lie- } \\
\text { gende körperliche Krank- } \\
\text { heit verursacht oder nicht }\end{array}$ & $\begin{array}{l}\text { Einfach, phänomenolo- } \\
\text { gisch, hochreliabel, hoch- } \\
\text { sensitiv }\end{array}$ & $\begin{array}{l}\text { Eventuell überinklusiv, } \\
\text { evtl. hohe Anzahl falsch } \\
\text { Positiver (niedrige Spezi- } \\
\text { fität), unklare Validität }\end{array}$ & $\begin{array}{l}\text { Zu empfehlen für den klini- } \\
\text { schen Gebrauch, im Indivi- } \\
\text { dualfall zu modifizieren } \\
\text { durch klinisches Urteil }\end{array}$ \\
\hline Ätiologisch & $\begin{array}{l}\text { Ein Symptom im Hinblick } \\
\text { auf Diagnose nicht zählen, } \\
\text { wenn "klar infolge der me- } \\
\text { dizinischen Bedingung" }\end{array}$ & Theoretisch überzeugend & $\begin{array}{l}\text { Im klinischen Alltag } \\
\text { schwierige Kausalattribu- } \\
\text { tion auf reliable Art, un- } \\
\text { klare Validität }\end{array}$ & $\begin{array}{l}\text { Theoretisch überzeugend, } \\
\text { praktisch schwierig zu } \\
\text { implementieren }\end{array}$ \\
\hline Exklusiv & $\begin{array}{l}\text { Ausschluß von somatischen } \\
\text { Symptomen (z. B. Müdigkeit, } \\
\text { Appetitlosigkeit) aus der Liste } \\
\text { der depressiven Symptome }\end{array}$ & $\begin{array}{l}\text { Verringert mögliche kon- } \\
\text { fundierende Variablen; } \\
\text { höhere Spezifität; niedri- } \\
\text { ge Anzahl falsch Positiver }\end{array}$ & $\begin{array}{l}\text { Schwierigere Diagnose- } \\
\text { stellung, möglicherweise } \\
\text { hohe Anzahl falsch Negati- } \\
\text { ver (niedrige Sensitivität), } \\
\text { Vorenthalten einer wirk- } \\
\text { samen Therapie }\end{array}$ & $\begin{array}{l}\text { Vorteilhaft für Forschungs- } \\
\text { projekte, zu exklusiv für } \\
\text { den klinischen Gebrauch }\end{array}$ \\
\hline Substitutiv & $\begin{array}{l}\text { Ersetzen somatischer } \\
\text { Symptome (z. B. Müdigkeit, } \\
\text { Appetitlosigkeit) durch } \\
\text { kognitive Symptome (z. B. } \\
\text { Grübeln Entscheidungs- } \\
\text { schwäche) }\end{array}$ & Konzeptuell vernünftig & Unklar & $\begin{array}{l}\text { Konzeptuell vernünftig, } \\
\text { aber bisher nur wenige } \\
\text { empirische Erfahrungen }\end{array}$ \\
\hline
\end{tabular}


Die folgende Übersicht stellt wichtige Aspekte zur diagnostischen Spezifizierung dar.

Übersicht. Wichtige Aspekte zur diagnostischen Spezifizierung depressiv-ängstlicher Störungen bei körperlichen Erkrankungen

- Definierte somatische Erkrankung, Akuität vs. Chronizität der Erkrankung, Krankheitsstadium, Chancen einer kurativen oder palliativen Therapie, durchgeführte Therapie,

- Orientierung an somatischen Krankheiten und medikamentösen Substanzgruppen mit empirisch etablierter hoher Assoziation von depressiven und Angststörungen (s. Abschn. 62.3.2),

- symptomatologische Orientierung an den diagnostischen Kategorien von Major Depression, Panikstörung, generalisierter Angststörung, spezifischen Phobien und posttraumatischer Belastungsstörung unter Wahrung einer Schweregradsdimension (Anzahl der registrierbaren Symptome) und differentieller Bewertung der somatischen Symptome,

- eigenständige Beachtung von Suizidalität, Mißbrauch/Ab hängigkeit von Alkohol und psychotropen Substanzen,

- psychiatrische Eigen- und Familienanamnese,

- Beginn und Dauer der affektiven Verstimmung bzw. Angststörung im Hinblick auf die somatische Erkrankung und durchgeführte somatische Therapien,

- relevante psychosoziale Auslösesituationen,

- relevante intrapsychische, interpersonale und soziale Auswirkungen der somatischen Erkrankung,

- bekannte Therapieerfahrungen bei früheren depressiven oder Angstepisoden.

\section{Differentialdiagnose}

Differentialdiagnostisch ist in der akuten Auseinandersetzung mit und der anschließenden Verarbeitung der somatischen Erkrankung zunächst v. a. den sukzessiven Phasen eines Trauerprozesses mit Schock, Desorganisation, Verleugnung, Protest und Akzeptanz bzw. Resignation eine eigenständige Beachtung zu widmen. In der Tat lassen sich im klinischen Verlauf aber fließende Übergänge zu depressiven Störungen, aber auch posttraumatischen Belastungsstörungen ausmachen (Horowitz et al. 1997; Prigerson et al. 1997; Zisook et al. 1997).

Depressionssyndrome durch organische Erkrankung. Organische Zustände, die das klinische Bild einer Depression häufig imitieren können, sind metabolische Enzephalopathien bei Hypokaliämie, Hypoglykämie oder Hyperkalzämie, bei einem Organversagen von Leber, Niere oder Lunge, endokrine Störunge wie Hypothyreoidismus, Hyperkortisolismus oder paraneoplastische Syndrome (Parathoromon, Vasopressin, Methionin, Enkephalin, $\beta$-Endorphin), zerebrale Läsionen wie Tumoren, Metastasen, Hämorrhagien, Infarkte, Tumorkachexie sowie medikamenteninduzierte Effekte (Cassem 1990). Nicht selten imponiert psychopathologisch bei diesen als depressive Verstimmung verkannten $\mathrm{Zu}$ ständen ein hypoaktives Delir (Lipowski 1990).
Angstsymptome durch organische Erkrankung. Organische Zustände, die das klinische Bild unterschiedlicher Angststörungen imitieren können, sind in Tabelle 62.3 aufgeführt. Nicht selten gehen auch hyperaktivagitierte Delire mit heftigen Angstsymptomen einher (Lipowski 1990). Zu beachten ist, daß v. a. objektivierbare Herzerkrankungen wie koronare Herzkrankheit mit Angina pectoris, kardiale Arrhythmien, mikrovaskuläre Angina, idiopathische Kardiomyopathie, aber auch das harmlose Mitralklappenprolapssyndrom mit einer hohen Prävalenz von Panikstörungen assoziiert sind. Diese organischen Zustände müssen als Kausalbedingungen differentialdiagnostisch erkannt und primär behandelt werden (Zaubler u. Katon 1998).

\section{7}

\section{Therapie}

Bei jedem Patienten mit einer ernsthaften somatischen Erkrankung kommt dem ärztlichen Gespräch, der sensiblen Aufklärung und der auf die Bedürfnisse des kranken Menschen abgestimmten Informationsvermittlung in einer supportiv stützenden Arzt-Patienten-Beziehung die entscheidende Rolle zu. Spezifische Therapiemaßnahmen bei pathologischen Trauerreaktionen bzw. koexistenten depressiven Störungen und Angststörungen umfassen psychologische und biologische Ansätze.

\subsection{1}

\section{Psychologische Ansätze}

Jede psychologische Intervention bei Patienten mit akuter Erkrankung sollte zunächst kurzfristig sein und darauf zielen, den Patienten in seinen Copingfertigkeiten zu unterstützen und ihm die Anpassung an die unabänderliche Situation des Krankseins zu erleichtern. Psychologische Ansätze sollten hierbei nicht uniform bei jedem Patienten überlegt werden. Kurztherapeutische Interventionen erscheinen zunächst dann indiziert, wenn der Anpassungsprozeß in der Auseinandersetzung mit der Erkrankung erheblich problematisiert oder aber deutlich in die Länge gezogen ist und mit notwendigen diagnostischen und therapeutischen Maßnahmen negativ interferiert. Ein am Modell der traumatischen Streßreaktionen orientiertes Vorgehen kann hierbei sehr behilflich sein (Tabelle 62.6).

\section{Psychotherapeutische Strategien}

Für depressive oder ängstliche Patienten mit somatischen Krankheiten v. a. bei chronischem Verlauf existieren eine Fülle von psychotherapeutischen Strategien, die z.T. psychodynamisch orientiert, z. T. kognitiv-verhaltenstherapeutisch ausgerichtet sind und als Einzel- 
Tabelle 62.6. Klassifikation von psychotherapeutischen Behandlungsansätzen bei traumatischen Belastungssyndromen (Horowitz 1976) von körperlich kranken Patienten. (Nach Levenson u. Hales 1993)

\begin{tabular}{|c|c|c|}
\hline Prozesse & Verleugnungsphase & Intrusionsphase \\
\hline $\begin{array}{l}\text { Veränderung der } \\
\text { Kontrollprozesse }\end{array}$ & $\begin{array}{l}\text { Reduktion der Kontrollvorgänge } \\
\text { Interpretation von dominanten Abwehrmechanismen, } \\
\text { Hypnose, Suggestion } \\
\text { Aufdeckende Interpretationen }\end{array}$ & $\begin{array}{l}\text { Aktives Angebot von Kontrollhilfen } \\
\text { Strukturierung von Zeit und Ereignissen, } \\
\text { stellvertretende Übernahme von Ich-Funktionen } \\
\text { Reduktion der externen Reizvielfalt } \\
\text { Schaffung von Ruhebedingungen } \\
\text { Erlaubnis zu Idealisierung und Abhängigkeit }\end{array}$ \\
\hline $\begin{array}{l}\text { Veränderung der } \\
\text { Informations- } \\
\text { verarbeitung }\end{array}$ & $\begin{array}{l}\text { Ermutigung zu Abreaktion } \\
\text { Ermutigung zu Detailbeschreibung } \\
\text { Einsatz von Bildern in der Phantasie, } \\
\text { Inszenierungen (z.B. Rollenspiel) }\end{array}$ & $\begin{array}{l}\text { Entfernung von quälenden Erinnerungsstücken } \\
\text { aus der unmittelbaren Umgebung } \\
\text { Differenzierung von Realität und Phantasie, } \\
\text { vergangenen und gegenwärtigen Selbst- und } \\
\text { Objektbildern } \\
\text { Unterdrückung der Denkvorgänge (z.B. durch } \\
\text { sedierende Medikation) }\end{array}$ \\
\hline $\begin{array}{l}\text { Veränderung der } \\
\text { Emotions- } \\
\text { verarbeitung }\end{array}$ & $\begin{array}{l}\text { Ermutigung zu Katharsis } \\
\text { Erforschung emotionaler Erlebnisaspekte } \\
\text { Ermutigung zu emotionalen Beziehungen }\end{array}$ & $\begin{array}{l}\text { Unterstützung } \\
\text { Evozieren anderer Emotionen } \\
\text { Desensibilisierungsmaßnahmen } \\
\text { Entspannungsverfahren, Biofeedback }\end{array}$ \\
\hline
\end{tabular}

verfahren oder aber im Gruppensetting durchgeführt werden (Gore-Felton u. Spiegel 2000; Levenson et al. 2000; Yates u. Bowers 2000). Die Anzahl der vorliegenden Studien, die sich auf ein kontrolliertes Vergleichsdesign mit Randomisierung stützen können, ist aber nach wie vor sehr bescheiden (Guthrie 1996). Hierbei sind psychosoziale bzw. psychotherapeutische Interventionen zu unterscheiden, die bei körperlich kranken Patienten auf eine Verbesserung des psychosozialen Outcome und des psychopathologischen Status, auf eine Verbesserung medizinischer Outcomevariablen, oder aber auf eine Reduktion der gesundheits- bzw. krankheitsbezogenen Kosten zielten (Levenson 1992).

Als generelle Bewertung kann gelten, daß die überwiegende Mehrheit dieser Studien günstige Effekte zeigte (Spiegel u. Lazar 1997). Trotzdem ist in Einzelstudien eine überzeugende Wirkung dieser flankierenden psychotherapeutischen Maßnahmen nicht nachweisbar gewesen.

Es besteht v.a. nur ein sehr geringer empirischer Beleg dafür, psychotherapeutische Interventionen ohne klare Indikation und präzise therapeutische Zielsetzung unterschiedslos bei allen Patienten einzusetzen (Guthrie 1996).

\section{Psychotherapeutische Intervention bei Krebspatienten}

Als Beispiel für psychotherapeutischen Interventionen sollen empirische Studien bei Krebspatienten kurz aufgeführt werden. Psychosoziale und psychotherapeutische Ansätze können im Gesamtbehandlungsplan eines Krebspatienten mittlerweile eine unverzichtbare Rolle beanspruchen (Fawzy et al. 1998). Auf 4 empirisch erprobte Basiskomponenten in solchen Verfahren ist hinzuweisen:

- soziale Unterstützung gegen soziale Isolation,

- emotionaler Ausdruck v.a. starker negativer Affekte,
- kognitive Umstrukturierung ungünstiger Denkschemata in der Verarbeitung der Krebserkrankung sowie

- Training von Copingstrategien.

Psychotherapeutische Behandlungen können Angst, Depressivität und Schmerzintensität effektiv reduzieren.

In 3 randomisierten Studien ließ sich zudem eine signifikante Verlängerung der Überlebenszeiten für Patienten mit Mammakarzinomen, Lymphomen und malignen Melanomen objektivieren (Spiegel et al. 1989; Richardson et al. 1990; Fawzy et al. 1993) Als mögliche Vermittlungsmechanismen dieses erstaunlichen Befunds werden ein verändertes Gesundheitsverhalten, eine bessere Inanspruchnahme ärztlicher und rehabilitativer Maßnahmen sowie endokrine und immunologische Veränderungen diskutiert (Spiegel 1994).

Anderseits sind diese positiven Effekte auf die Überlebenszeit nicht in allen Studien belegbar wie z. B. bei Gellert et al. (1993). In einer Studie an Frauen mit Mammakarzinom am Bristol Cancer Help Centre stellte sich sogar unerklärlich eine negative Korrelation zwischen Teilnahme an einem psychologischen Unterstützungsprogramm und der Überlebenszeit dar (Bengal et al. 1990).

\subsection{2}

\section{Biologische Ansätze}

Ein psychopharmakologischer Ansatz bei somatisch kranken Patienten mit depressiv-ängstlichen Störungen bezieht sich vorrangig auf den Einsatz von Antidepressiva. Er sollte immer dann diskutiert werden, wenn der klinische Schweregrad der depressiven Verstimmung den diagnostischen Kriterien einer Major Depression entspricht und notwendige internistisch-medikamen- 
töse Korrekturen keine zufriedenstellende symptomatische Erleichterung erbracht haben.

Eine Psychopharmakotherapie ist nicht im Wider- streit mit psychologischen Interventionen, sondern in einer notwendiger Ergänzung zu ihnen zu sehen.

Prominente Angstsymptome, besonders auch bei suizidalen Krisen können eine zeitlich limitierte Verordnung von Benzodiazepinen erfordern. Diese können in aller Regel mit guter Sicherheit und hoher Wirksamkeit eingesetzt werden (Kapfhammer 1995; s. Übersicht).

Übersicht. Der Einsatz von Benzodiazepinen bei somatischen Erkrankungen. (Nach Kapfhammer 1995)

- Kardiovaskuläre Erkrankungen

- Nebenwirkungen auf Reizleitung und Leistung des Herzens vernachlässigbar,

- protektiver Schutz bei Herzinfarktpatienten durch Verringerung der angstassoziierten Tachykardien und $\mathrm{Ar}$ rhythmien,

- möglicher Anstieg des Digoxinspiegels durch Interaktion,

- günstiges Adjuvans bei streßreagiblem Hypertonus, bei implantiertem Kardioversionsdefibrillator und künstlichen Herzklappen mit beängstigend lauter Geräuschbildung.

- Pulmonale Erkrankungen

- günstiges Adjuvans zur Koupierung akuter Asthmaanfälle,

- möglicher atemdepressiver Effekt bei chronisch obstruktiven Atemwegserkrankungen, jedoch durchaus günstig bei chronischem Lungenemphysem und niedrigem oder normalem $\mathrm{pCO}_{2}$, aber verringertem $\mathrm{pO}_{2}$ („pink puffers"),

- Kontraindikation bei Schlafapnoe!,

- nach längerfristiger respiratorischer Unterstützung und intensivmedizinischer Behandlung mit Fentanyl und Midazolam oft Reboundphänomene mit schweren Panikzuständen: Erleichterung der Entwöhnung durch zeitlich überlappende Gabe eines länger wirksamen Benzodiazepins, z. B. Dikaliumclorazepat.

- Gastrointestinale Erkrankungen

- passagerer Einsatz bei assoziierten Angst- und Ärgeraffekten sinnvoll sowie bei anxiogenen Wirkungen von Internistika, z. B. Steroiden, Sulfasalazinen, Immunsuppressiva.

- Hepatorenale Erkrankungen

- wesentliche Beeinflussung der Metabolisierung und Elimination, Kumulation aktiver Metabolite mit Nebenwirkungen einer extremen Sedierung, Ataxie und Sturzneigung: Dosisreduktion,

- Clonazepam, Lorazepam, Oxazepam, Temazepam ohne aktive Metabolitenbildung.

- Ältere Patienten

- reduzierte Metabolisierungsleistungen, veränderte pharmakodynamische Bedingungen: erhöhte Nebenwirkungsempfindlichkeit: Sturzgefahr, paradoxe Effekte, kognitive Dysfunktionen, depressive Verstimmungen.

- Unter Triazolam erhöhte Rate zerebrovaskulärer Zwischenfälle (Hemmung des Plättchen-Aggregations-Faktors/PAF) beobachtet.

\section{Nutzen-Risiko-Abwägung}

Eine antidepressive Medikation bei somatischen Erkrankungen wird in der Literatur v.a. im Hinblick auf eine Nutzen-Risiko-Bewertung kontrovers diskutiert.
Ernste Nebenwirkungen gerade bei somatischen Krankheitsprozessen begrenzen nicht selten eine suffiziente antidepressive Behandlung (Popkin et al. 1985; Schwartz et al. 1988, 1989). Es gibt mittlerweile aber eine Reihe von Erfahrungsberichten (Cassem 1988; Rodin u. Voshart 1986; Winokur et al. 1988) wie auch von kontrollierten Studien über den erfolgversprechenden Einsatz von Antidepressiva bei definierten somatischen Erkrankungen in einem stationären Behandlungsrahmen (Andersen et al. 1980: M. Parkinson - Nortriptylin; Borson et al. 1992: chronische obstruktive Lungenerkrankung - Nortriptylin; Costa et al. 1985: Mammakarzinom - Mianserin; Evans et al. 1997: ältere Patienten mit diversen internistischen Erkrankungen - Fluoxetin; Light et al. 1986: chronische obstruktive Lungenerkrankung Doxepin; Lipsey et al. 1984: Apoplex - Nortriptylin; Lustman et al. 1997: Diabetes mellitus - Nortriptylin; Rifkin et al. 1985: unterschiedliche internistische Erkrankungen - Trimipramin; Robertson u. Trimble 1985: Epilepsie - Amitriptylin/Nomifensin; Roose et al. 1998: ischämische Herzerkrankung - Paroxetin/Nortriptylin; Veith et al. 1982: Herzerkrankung - Imipramin/Doxepin).

\section{Dosierung}

Grundsätzlich spricht wenig dafür, daß bei der antidepressiven Behandlung von somatisch kranken Patienten im Vergleich zu nicht körperlich beeinträchtigten Patienten niedrigere Dosierungen zu einem Therapieerfolg führten (Fava u. Sonnino 1996). Ausnahmen bilden lediglich Patienten mit fortgeschrittenen Lebererkrankungen oder mit einer terminalen Niereninsuffizienz. Auch bei älteren Patienten sind veränderte Metabolisierungsbedingungen zu beachten (Fava et al. 1988; Koenig u. Breitner 1990; Koenig et al. 1997).

\section{Substanzklassen der Antidepressiva}

Neben den traditionellen Substanzklassen der heterozyklischen Antidepressiva und den irreversiblen MAOHemmern ist die Entwicklung v. a. durch die Einführung von 2 Substanzklassen vorangetrieben worden. Es sind dies die selektiv wirksamen Serotoninwiederaufnahmehemmer (SSRI) einerseits sowie die selektiven und reversiblen MAO-Hemmer (RIMA) andererseits. Zusätzlich wurden als neue Präparate Venlafaxin (duales Wirkprinzip in der selektiven Wiederaufnahmehemmung von Serotonin und Nordrenalin, SSNI), Mirtazapin (noradrenerges und spezifisch serotonerges Antidepressivum, NaSSA), Nefazodon und Reboxetin (selektive Noradrenalinwiederaufnahmehemmung) eingeführt.

Im Konsiliarbereich werden ferner in letzter Zeit wieder verstärkt amphetaminerge Stimulanzien (Methylphenidat, Dextroamphetamin, Pemolin) eingesetzt. Sie sind als antidepressive Behandlungsstrategie bei somatisch kranken Patienten immer dann zu erwägen, wenn eine Kontraindikation für andere Antidepressiva vorliegt, oder aber ein rascher positiver Effekt z. B. zur früh- 
zeitigen Mobilisierung eines anergen Patienten angestrebt wird (Satel u. Nelson 1989). Die Rate der Nebenwirkungen ist insgesamt relativ niedrig. Zu beachten sind aber eine mögliche Agitiertheit, Angst, Schlaflosigkeit sowie eine depressive Verstimmung bei Absetzen nach einer längerfristigen Applikation (Masand et al. 1991).

\section{Bedingungen für die Wahl der Medikation}

Eine praktische Orientierung für den Einsatz von Antidepressiva bei somatisch kranken Patienten läßt sich auf 3 fache Weise erzielen (Kapfhammer 1998):

- durch eine detaillierte Kenntnis möglicher Nebenwirkungen einzelner Substanzklassen,

- durch ein detailliertes Wissen der hepatischen Metabolisierung v.a. im Zytochrom-P-450-System sowie bedeutsamer Interaktionen mit anderen Internistika,

- durch die Beachtung spezieller pathophysiologischer Grundbedingungen bei definierten Krankheitsbildern.

\section{Nebenwirkungen und Interaktionen}

Vergleicht man die neu eingeführten Antidepressiva mit den alt bewährten Präparaten, um eventuelle Fortschritte in der Pharmakotherapie depressiver Störungen zu ermessen, so genügt ein bloßer Wirksamkeitsvergleich nicht. Es ist vielmehr eine multidimensionale Vergleichsanalyse anzustellen. Diese beinhaltet nicht nur die Aspekte der globalen Wirksamkeit, des klinischen Wirkprofils und der Schnelligkeit des Wirkungseintritts, sondern berücksichtigt im gleichen Maße auch die globale Verträglichkeit, das typische Nebenwirkungsspektrum, das Letalitätsrisiko bei Intoxikationen und relevante Interaktionen mit anderen Pharmaka. Eine Beurteilung der einzelnen antidepressiven Substanzklassen stützt sich also auf eine differenzierte Nutzen-Risiko-Kalkulation (Möller 1996).

Die beiden hauptsächlichen Substanzklassen - Trizyklika und SSRI - weisen unterschiedliche Nebenwirkungsprofile auf, die eine Reihe von Vorteilen auf Seiten der SSRI vermuten lassen (s. Kap. 27). Auch die RIMA und die anderen neuen Antidepressiva beweisen insgesamt ein recht günstiges Nebenwirkungsprofil. Hinsichtlich des Letalitätsrisikos bei versehentlicher oder suizidaler Einnahme von toxischen Dosierungen erweisen sich die Trizyklika gegenüber den SSRI als ebenfalls nachteilig (Henry 1989).

Es ist sicherlich noch zu früh, die erst jüngst eingeführten Antidepressiva schon hinsichtlich ihres Nebenwirkungsprofils bei somatischen Risikogruppen zu bewerten. Einige interessante Alternativen deuten sich aber auch bei ihnen an (Kapfhammer 1998, 1999; Brown et al. 2001).

Tabelle 62.7 faßt internistisch relevante unerwünschte Arzneimittelnebenwirkungen der einzelnen antidepressiven Substanzgruppen zusammen. Tabelle 62.8 gibt einen Einblick in die differentielle hepatische Meta- bolisierung der Antidepressiva, speziell der SSRI durch das oxidative Zytochrom-P-450-System.

Die folgenden 3 Übersichten fassen wichtige Interaktionen der Antidepressiva mit anderen in der somatischen Medizin häufig eingesetzten Pharmaka zusammen.

Übersicht. Klinisch bedeutsame Interaktionen von Trizyklika mit anderen Pharmaka

- Typ-I-A/C Antiarrhythmika (Chinidin, Procainamid, Flecainid): mögliche Verlängerung der kardialen Überleitungszeit, erhöhtes Risiko für proarrhythmische Effekte,

- Phenothiazine: verlängertes QT-Intervall, erhöhte Plasmaspiegel der trizyklischen $\mathrm{AD}$,

- Disulfiram, Methylphenidat, Cimetidin, Fenfluramin, Allopurinol, Metronidazol, Chloramphenicol, Isoniazid, Sulfonamide: erhöhte Plasmaspiegel der trizyklischen $\mathrm{AD}$,

- orale Kontrazeptiva, Äthanol, Barbiturate, Phenytoin, Glutethimid, Methaqualon, Chloraldurat, Diphenhydramin, Nicotin, Griseofulvin, Colestyramin: erniedrigte Plasmaspiegel der trizyklischen $\mathrm{AD}$,

- Reserpin, Guanethidin, Clonidin, Methyldopa, Guanfacin: hypotensiver Effekt verringert,

- Prazosin, Hydralazin, Minoxidil, Captopril, Nifedipin, Thiazid- und Schleifendiuretika: hypotensiver Effekt verstärkt,

- Phenprocoumon (Marcumar): erhöhtes Blutungsrisiko bei verlängerter Prothrombinzeit,

- Antazida, L-Dopa: verringerte intestinale $\mathrm{AD}$-Absorption,

- Anticholinergika: verstärkter anticholinerger Effekt,

- Carbamazepin: erhöhte Kardiotoxizität möglich, verringerte AD-Plasmaspiegel, erhöhte Spiegel der AD-Metaboliten,

- Halothan, Pancuronium: Tachykardie, verstärkte Arrhythmien,

- L-Thyroxin: Tachykardie, verstärkte Arrhythmien,

- parenterale Sympathomimetika (z. B. Epinephrin, Norepinephrin): hypertensive Reaktionen möglich.

Übersicht. Klinisch bedeutsame Interaktionen von MAOHemmern mit anderen Pharmaka

- Pethidin: mögliche tödliche Zwischenfälle!

- L-Dopa, Methyldopa, Dopamin, Buspiron, Guanethidin, trizyklische AD, Carbamazepin: Blutdruckanstieg,

- direkte Sympathomimetika (Epinephrin, Norepinephrin, Isoproterenol): Blutdruckanstieg,

- indirekte Sympathomimetika (Kokain, Amphetamin, Tyramin, Methylphenidat, Ephedrin, Phenylpropanolamin), direkte/indirekte Sympathomimetika (Pseudoephedrin, Metaraminol, Phenylephedrin): hypertensive Krise,

- serotonerge Substanzen (Clomipramin, L-Tryptophan, SSRI): „Serotoninsyndrom“ (Ataxie, Nystagmus, Verwirrtheit, Fieber, Tremor),

- Koffein, Theophyllin, Aminophyllin: leichter Blutdruckanstieg,

- orale Antidiabetika/Insulin: verstärkte Blutzuckersenkung,

- Succinylcholin: verlängerte Wirkzeit,

- Antihypertonika (Diuretika, Propranolol, Prazosin, Kalziumkanalblocker): verstärkter hypotensiver Effekt. 
Tabelle 62.7. Ausgewählte Antidepressiva: Haupteffekte in aminergen Neurotransmittersystemen und Nebenwirkungsprofil. (Nach Baldessarini 1996)

\begin{tabular}{|c|c|c|c|c|c|c|c|}
\hline $\begin{array}{l}\text { Substanz- } \\
\text { gruppen }\end{array}$ & $\begin{array}{l}\text { Vermittlung } \\
\text { der antidepres- } \\
\text { siven Effekte }\end{array}$ & Sedierung & $\begin{array}{l}\text { Anticholinerge } \\
\text { Effekte }\end{array}$ & $\begin{array}{l}\text { Hypotension- } \\
\text { wirkung }\end{array}$ & $\begin{array}{l}\text { Kardiale } \\
\text { Wirkung }\end{array}$ & $\begin{array}{l}\text { Krampf- } \\
\text { anfälle }\end{array}$ & $\begin{array}{l}\text { Gewichts- } \\
\text { zunahme }\end{array}$ \\
\hline \multicolumn{8}{|l|}{ Heterozyklika } \\
\hline \multicolumn{8}{|l|}{ Tertiäre Amine } \\
\hline Amitriptylin & NA, 5-HT & +++ & +++ & +++ & +++ & ++ & ++ \\
\hline Clomipramin & $\mathrm{NA}, 5-\mathrm{HT}$ & ++ & +++ & ++ & +++ & +++ & + \\
\hline Doxepin & NA, 5-HT & +++ & ++ & +++ & ++ & ++ & ++ \\
\hline Imipramin & $\mathrm{NA}, 5-\mathrm{HT}$ & ++ & ++ & ++ & +++ & ++ & ++ \\
\hline Trimipramin & $\mathrm{NA}, 5-\mathrm{HT}$ & +++ & +++ & ++ & +++ & ++ & ++ \\
\hline \multicolumn{8}{|l|}{ Sekundäre Amine } \\
\hline Desipramin & NA & $0 /+$ & + & + & ++ & + & + \\
\hline Nortriptylin & NA & + & + & + & ++ & + & + \\
\hline Protriptylin & NA & $0 /+$ & ++ & + & +++ & ++ & + \\
\hline Maprotilin & NA & ++ & ++ & ++ & ++ & +++ & + \\
\hline \multicolumn{8}{|c|}{ Serotoninwiederaufnahmehemmer } \\
\hline Fluvoxamin & 5-HT & $0 /+$ & 0 & 0 & 0 & 0 & 0 \\
\hline Fluoxetin & 5-HT & $0 /+$ & 0 & 0 & 0 & 0 & 0 \\
\hline Paroxetin & $5-\mathrm{HT}$ & $0 /+$ & 0 & 0 & 0 & 0 & 0 \\
\hline \multicolumn{8}{|c|}{ Atypische Antidepressiva } \\
\hline Trazodon & 5-HT & +++ & 0 & ++ & $0 /+$ & 0 & + \\
\hline \multicolumn{8}{|l|}{ MAO-Hemmer } \\
\hline Tranylcypromin & NA, 5-HT, DA & + & 0 & ++ & 0 & 0 & + \\
\hline
\end{tabular}

NA noradrenerg, 5-HT serotonerg, $D A$ dopaminerg; 0 fehlend, + gering, ++ mäßig, +++ ausgeprägt.

Tabelle 62.8. Antidepressiva als Inhibitoren wichtiger im oxydativen Zytochrom-P-450 System involvierter Enzymsubsysteme. (Nach Brøsen 1996; Ereshefsky 1996)

\begin{tabular}{|c|c|c|c|c|}
\hline Enzymsubsysteme & CYP 1A1/1A2 & CYP 2C9/19 & CYP 2D6 & CYP 3 A4 \\
\hline Modellsubstrate & Phenacetin & Mephenytoin & $\begin{array}{l}\text { Spartein } \\
\text { Debrisoquin }\end{array}$ & $\begin{array}{l}\text { Ketoconazol } \\
\text { Erythromycin } \\
\text { Midazolam }\end{array}$ \\
\hline Substrate & $\begin{array}{l}\text { Theophyllin } \\
\text { Propranolol } \\
\text { Marcumar } \\
\text { Trizyklika } \\
\text { Clozapin } \\
\text { Koffein } \\
\text { Olanzapin }\end{array}$ & $\begin{array}{l}\text { Diazepam } \\
\text { Marcumar } \\
\text { Phenytoin } \\
\text { Trizyklika } \\
\text { Clozapin } \\
\text { Barbital } \\
\text { Moclobemid }\end{array}$ & $\begin{array}{l}\text { Antiarrhythmika } \\
\beta \text {-Blocker } \\
\text { Kodein } \\
\text { SSRI } \\
\text { Clozapin } \\
\text { Haloperidol } \\
\text { Risperidon } \\
\text { Trizyklika } \\
\text { Olanzapin }\end{array}$ & $\begin{array}{l}\text { Carbamazepin } \\
\text { Benzodiazepine } \\
\text { Trizyklika } \\
\text { Cyclosporine } \\
\text { Nifedipin }\end{array}$ \\
\hline Inhibitoren & $\begin{array}{l}\text { Fluvoxamin } \\
\text { Cimetidin }\end{array}$ & $\begin{array}{l}\text { Fluoxetin } \\
\text { Fluvoxamin } \\
\text { Sertralin }\end{array}$ & $\begin{array}{l}\text { Fluoxetin } \\
\text { Paroxetin } \\
\text { Sertralin } \\
\text { Venlafaxin } \\
\text { Fluphenazin } \\
\text { Thioridazin } \\
\text { Citalopram } \\
\text { Cimetidin }\end{array}$ & $\begin{array}{l}\text { Fluoxetin } \\
\text { Fluvoxamin } \\
\text { Sertralin } \\
\text { Cimetidin }\end{array}$ \\
\hline Induktoren & Rauchen & Rifampicin & Unbekannt & $\begin{array}{l}\text { Carbamazepin } \\
\text { Barbiturate, Phenytoin }\end{array}$ \\
\hline Polymorphismen & Möglich & $\mathrm{Ja}$ & Ja & Unklar \\
\hline
\end{tabular}


Übersicht. Klinisch bedeutsame Interaktionen von SSRI mit anderen Pharmaka

- Digitoxin: Verdrängung aus Proteinbindung, verstärkte Digitoxinwirkung,

- Antiarrhythmika (Propafenon, Flecainid): erhöhte Plasmaspiegel der Antiarrhythmika,

- Phenprocoumon (Marcumar): erhöhtes Blutungsrisiko bei verlängerter Prothrombinzeit,

- Cyclosporin: erhöhte Cyclosporinspiegel,

- Carbamazepin: erhöhte Carbamazepinspiegel, erhöhte Neurotoxizität,

- Alprazolam, Diazepam: verlängerte HWZ,

- trizyklische AD, Trazodon, Neuroleptika: erhöhte Plasmaspiegel,

- Neuroleptika: verstärkte EPMS,

- MAO-Hemmer, Fenfluramin, L-Tryptophan, Sumatriptan: "Serotoninsyndrom“,

- Lithium: erhöhte Neurotoxizität,

- Pentazocin: schwere Erregung, u. a. neurotoxische Zeichen,

- orale Antidiabetika/Insulin (v. a. Sulfonylharnstoffe): verstärkte Blutzuckersenkung.

\subsection{3}

\section{Einsatz von Antidepressiva \\ bei definierten internistischen Erkrankungen}

\section{Zustand nach Myokardinfarkt}

Der Einsatz von trizyklischen Antidepressiva 6 Wochen nach einem unkompliziertem Myokardinfarkt gilt als unbedenklich. Diese Zeitspanne entspricht der vermutlichen Dauer bis zur Stabilisierung bzw. Heilung des infarzierten Myokards. Eine Gabe von trizyklischen Antidepressiva vor Ablauf dieses Zeitraumes wurde früher bei unkompliziertem Postinfarktverlauf für vertretbar gehalten. Die Ergebnisse der CAST-Studie, die eine höhere Mortalitätsrate von Postmyokardinfarktpatienten unter Klasse-I-Antiarrhythmika auch über diesen Zeitraum hinaus belegen, raten aber insgesamt zu einer vorsichtigen Grundeinstellung hinsichtlich des Einsatzes von trizyklischen Antidepressiva bei diesen Patienten. Andererseits sprechen experimentelle Daten auch dafür, daß unter diesen Substanzen die myokardiale Reperfusion durch verstärkte Bildung von Kollateralen gefördert werden kann (Manoach et al. 1989).

Notwendigkeit der Behandlung. Die hohe Prävalenz von behandlungsbedürftigen depressiven Verstimmungen nach überstandenem Myokardinfarkt illustriert die Notwendigkeit, für diese Patienten ein sicheres antidepressiv wirkendes Medikament zur Hand zu haben. Bei nicht behandeltem depressivem Syndrom kann eine mögliche Verzögerung des weiteren Gesundungsprozesses resultieren. Ein Zusammenhang mit einer erhöhten Komplikationsrate und Mortalität durch kardiovas- kuläre Ereignisse wird diskutiert (Dalack u. Roose 1990; Frasure-Smith 1991).

Nutzen-Risiko-Kalkulation. In jedem Fall sollte eine Entscheidung für den Einsatz von Trizyklika auf einer individuellen Nutzen-Risiko-Kalkulation erfolgen. Hierbei muß auch bedacht werden, daß es bei Infarktpatienten, die bereits zuvor unter antidepressiver Medikation standen, durch Absetzen der trizyklischen Antidepressiva zu einem Rezidiv der depressiven Störung kommen kann. Damit einhergehend erscheint eine Verschlechterung kardialer Parameter möglich. So kann es gerade bei abruptem Absetzen zu gefährlichen Arrhythmien kommen (Regan et al. 1989).

Interaktionen und Nebenwirkungen. Eine Behandlung mit trizyklischen Antidepressiva nach Myokardinfarkt geht Hand in Hand mit einer optimalen Behandlung möglicher Folgezustände wie beispielsweise einer Herzinsuffizienz. Es ist darauf zu achten, daß die Elektrolyte, v. a. Kalium, im Normbereich liegen. Die ungünstige Interaktion von Trizyklika und Antiarrhythmika vom Chinidintyp muß vermieden werden (Levenson $\mathrm{u}$. Friedel 1985). Bei der Wahl des Antidepressivums sollte man auf die unterschiedlichen Auswirkungen auf die atrioventrikuläre und ventrikuläre Reizbildung und Reizleitung (vorteilhaft: Mianserin) achten sowie auf orthostatische Regulationsstörungen (vorteilhaft: Nortriptylin). Unter kontinuierlicher Kontrolle von Herzrhythmus und Blutdruck, und unter Beachtung von möglichen Nebenwirkungen und Interaktionen mit anderen Pharmaka, kann in vorsichtigen Schritten eine mittlere Tagesdosierung angestrebt werden.

Andere Antidepressiva. Die SSRI, die reversiblen und selektiven MAO-Inhibitoren, Venlafaxin, Mirtazapin, Reboxetin und Nefazoden zeichnen sich durch ein insgesamt günstigeres kardiales Nebenwirkungsprofil aus. Es muß aber betont werden, daß detaillierte klinische Erfahrungen mit dem Einsatz dieser Präparate bei Myokardinfarktpatienten nicht existieren. Als Vorteil von Fluoxetin kann angesehen werden, daß es Ärgerund Feindseligkeitsaffekte bei einer Depression reduzieren kann. Hinsichtlich eines möglichen Zusammenhangs von Serotonin, Ärger und kardiovaskulärem Risiko ergäbe sich deshalb für Fluoxetin eine interessante Indikation (Fava et al. 1993).

Benzodiazepine. Die Akutphase eines Herzinfarkts kann problemlos durch der Einsatz von Benzodiazepinen wie Lorazepam oder Alprazolam überbrückt werden. Beiden Präparaten kann eine milde stimmungsaufhellende Wirkkomponente und eine gute Streßprophylaxe zugesprochen werden. Bei längerfristiger Applikation muß aber die Gewöhnungsgefahr beachtet werden. 
Stimulanzien. Unter stationären Bedingungen ist in der Anfangsphase sehr wohl auch eine niedrig dosierte Therapie mit amphetaminergen Stimulanzien (Beispiel: Methylphenidat) mit langsamer Höherdosierung und Kontrolle möglicher Nebenwirkungen als probate Therapiealternative zu erwägen. Gerade bei anergen Syndromen, die einer gewünschten Rehabilitation eines Patienten negativ entgegenstehen, kann z.B. Methylphenidat versucht werden. Nach einer initialen Gabe von 2,5 mg kann die Dosis meistens ohne wesentliche Probleme innerhalb weniger Tage auf 10-20 mg gesteigert werden. Von Vorteil ist es, die Tagesdosis auf 1-2 Einzeldosen aufzuteilen. Ein Therapieeffekt zeichnet sich in aller Regel bereits nach wenigen Tagen ab, so daß über Fortführung oder Abbruch der Behandlung meist schon nach kurzer Zeit entschieden werden kann (Chiarello u. Cole 1987).

\section{Rhythmusstörungen}

\section{QT-Verlängerung und WPW-Syndrom}

Eine strenge Kontraindikation für den Einsatz von trizyklischen Antidepressiva besteht, wenn bereits vor Therapie eine signifikante QT-Verlängerung vorliegt, oder aber nach Behandlungsbeginn auftritt.

Als oberste Grenze kann 0,44 s für das korrigierte QT-Intervall (QTc) angegeben werden (Stoudemire u. Atkinson 1988). Patienten mit einem WPW-Syndrom sind ebenfalls als eine spezielle Risikogruppe zu behandeln, bei der Trizyklika maligne Arrhythmien auslösen können, v.a. wenn eine sehr kurze refraktäre Periode von unter $0,27 \mathrm{~s}$ besteht.

Bei Patienten mit diesen Rhythmusanomalien sollten keine Trizyklika verordnet werden und statt dessen auf Präparate aus anderen Wirkklassen ausgewichen werden. Doch auch hier sollte eine verstärkte kardiologische Überwachung erfolgen. Ist nur vorrübergehend eine symptomatische Entlastung erforderlich, können auch Benzodiazepine verordnet werden. Bei schwerwiegenden Depressionen ist eine Elektrokrampftherapie (EKT) zu diskutieren. Weder Benzodiazepine noch eine EKT induzieren QT-Veränderungen. Auch MAO-Hemmer besitzen hier eine günstigeres Nebenwirkungsprofil. Die orthostatischen Nebenwirkungen und die zahlreichen Medikamenteninteraktionen sind allerdings zu beachten.

\section{Schenkelblock}

Patienten mit sowohl einem Rechts- als auch einem Linksschenkelblock haben ein gewisses Risiko, unter Trizyklika einen kompletten AV-Block zu entwickeln. Hierbei ist es nicht möglich, dieses Risiko abzuschätzen (Roose et al. 1987). Bei einem bifaszikulären oder inkompletten trifaszikulären Block, einem alternierendem Schenkelblock, einem AV-Block 2. oder 3. Grades kann der Einsatz von Trizyklika nur vertreten werden, wenn ein Schrittmacher implantiert wurde (Stoudemire u. Atkinson 1988). Präparate aus anderen Wirkklassen gelten bei diesen Erregungsleitungsstörungen nicht als kontraindiziert. Die Patienten müssen aber in jedem Fall unter Antidepressiva engmaschig überwacht werden.

\section{AV-Block}

Bei Patienten mit einem AV-Block 1. Grades (PR-Intervall $>0,20$ s) gilt die Behandlung mit Trizyklika als unproblematisch. In mehreren Studien konnte die nach theoretischen Überlegungen denkbare Induktion eines höhergradigen AV-Blocks nicht bestätigt werden (Dietch u. Fine 1990; Roose et al. 1987).

\section{Herzinsuffizienz}

Trizyklika, aber auch Antidepressiva aus anderen Wirkklassen können mit guter Sicherheit bei herzinsuffizienten Patienten eingesetzt werden. Voraussetzung ist selbstverständlich eine ausreichende internistische Stabilisierung. Aus klinischer Sicht ist jedoch zu bedenken, daß bei einer bestehenden Herzinsuffizienz das Risiko einer trizyklikainduzierten orthostatischen Hypotension erheblich ansteigen kann. Nortriptylin erscheint unter den trizyklischen Antidepressiva am ehesten geeignet und besitzt möglicherweise auch gegenüber den SSRI gewisse Vorteile.

Bei einer Herzinsuffizienz kommt es zu einer interstitiellen Flüssigkeitsretention und hierüber zu einem verminderten zentralen Verteilungsvolumen. Zudem können auch der hepatische Blutfluß und die enzymatische Aktivität in der Leber erheblich eingeschränkt sein. All diese Faktoren können zu höheren Plasmakonzentrationen der hepatisch metabolisierten Antidepressiva beitragen. Kleinere Tagesdosen sind deshalb ausreichend. Eine Plasmaspiegelbestimmung unter "steady-state“-Bedingungen ermöglicht eine bessere Orientierung.

\section{Erhöhter und erniedrigter Blutdruck}

Die meisten trizyklischen Antidepressiva, aber auch die klassischen MAO-Hemmer besitzen einen eigenständigen hypotensiven Effekt. SSRI, RIMA, Venlafaxin, Mirtazapin, Reboxetin und Nefazodon weisen diesbezüglich Vorteile auf. Bei Venlafaxin kann unter höherer Dosierung eine Blutdrucksteigerung auftreten.

Klinische Probleme können sich aus den Interaktionen von Trizyklika mit Antihypertonika ergeben. Die zu beobachtende Wirkung ist uneinheitlich. Einerseits kann die hypotensive Wirkung von Guanethidin, Methyldopa, Clonidin, Reserpin oder Guanfacin reduziert, andererseits kann der blutdrucksenkende Effekt von Thiazid- und Schleifendiuretika, Nifedipin, Minoxidil, Hydralazin, Prazosin oder Captopril verstärkt werden (Stoudemire et al. 1990). 
Beim Phäochromozytom sind Trizyklika und SSRI kontraindiziert (Fava et al. 1988).

Bei der Behandlung einer symptomatischen arteriellen Hypotonie ist zu beachten, daß adrenerge Kreislaufmittel und trizyklische Antidepressiva infolge einer Blockade der Adrenorezeptoren zu einem Überwiegen $\operatorname{der} \beta$-agonistischen Wirkung und hierüber zu einer Verstärkung der Hypotonie führen können. (Bandelow u. Rüther 1993). Für die klinische Praxis sind bei einer arteriellen Hypotonie Präparate vorzuziehen, die wie Nortriptylin weniger zu orthostatischer Dysregulation führen.

\section{Atemwegs- und Lungenerkrankungen}

Vor allem ältere Patienten mit Dyspnoe im Rahmen von respiratorischen Störungen scheinen besonders anfällig für depressive Verstimmungen zu sein, wobei dem gestörten Schlafrhythmus, der ausgeprägten funktionellen Behinderung und den möglichen hypoxischen Effekten eine besondere prädisponierende Rolle zugesprochen wird (Thompson u. Thompson 1984, 1993).

Trizyklische Antidepressiva können der Mehrzahl der Patienten mit pulmonalen Erkrankungen ohne zusätzliche Einschränkung gegeben werden. Bei gesunden Personen führen sie nicht zu einer Atemdepression. Allerdings liegen nur wenige empirische Daten für $\mathrm{Pa}-$ tienten mit einer chronischen obstruktiven Atemwegserkrankung vor. Theoretisch läßt ein zentraler wie auch ein peripherer anticholinerger Effekt neben der antidepressiven Wirkung auch eine günstige bronchodilatatorische Wirkung erwarten, andererseits kann dieser Effekt auch eine nicht erwünschte Zunahme der Herzfrequenz bewirken. Light et al. (1986) fanden für Doxepin gegenüber Plazebo nur geringfügige Vorteile, wohingegen Borson und Mitarbeiter (1992) für Nortriptylin durchaus positive Effekte fanden. Antiasthmatische Wirkungen werden dem Amitriptylin nachgesagt (Ananth 1974; Meares et al. 1971; Wilson 1974).

Ältere Patienten. Vor allem bei älteren Patienten muß darauf geachtet werden, daß eine Hypoxämie kardiale Arrhythmien begünstigt. Diese Situation kann durch die sedierende Komponente der Trizyklika ungünstig beeinflußt werden. Eine Kombination mit Benzodiazepinen führt zu einer weiteren Erhöhung dieses Risikos. Die anticholinergen Effekte von Trizyklika können zu einer Austrocknung der Bronchialschleimhaut führen, das Bronchialsekret zähflüssiger machen und dadurch das Abhusten von Schleim erschweren.

Patienten mit Allergien. In seltenen Fällen können tartrazinhaltige Farbstoffe (gelb, orange), die in der galenischen Aufbereitung einiger trizyklischen Präparate enthalten sind, einen Bronchospasmus provozieren. Besonders gefährdet erscheinen Patienten mit ei- nem aspirinsensitiven Asthma (Thompson u. Thompson 1993).

Die Gabe von MAO-Hemmern ist bei jenen Patienten kontraindiziert, die z.B. ephedrinhaltige Substanzen zum Abschwellen der Schleimhäute bei Rhinitis oder Sinusitis erhalten (Fava et al. 1988).

Neuere Antidepressiva. Für die in jüngerer Zeit entwickelten antidepressiven Präparate gibt es kaum zuverlässige Daten hinsichtlich ihres Einsatzes bei dieser Patientengruppe. Eine ungünstige Beeinflussung der Symptomatik von COPD-Patienten ist nicht bekannt. $\mathrm{Zu}$ beachten ist aber, daß der Hersteller von Fluoxetin als häufige Nebenwirkungen ( $>1 / 100$ Patienten) „Bronchitis", als nicht häufige Ereignisse (zwischen 1/100 und 1/1.000 Patienten) „Asthma, Hyperventilation und Pneumonie" und als seltene Effekte $(<1 / 1.000$ Patienten) „Apnoe und Hypoxie“ aufführt (Bass u. Colebatch 1992; Gonzalez et al. 1995; Rubey u. Lydiard 1994).

\section{Gastrointestinalen Erkrankungen}

Erkrankungen des Magen-Darm-Trakts gehen häufig mit psychischen Auffälligkeiten einher (Fava et al. 1985). Die pharmakologische Behandlung depressivängstlicher Störungen mit Antidepressiva orientiert sich zunächst am Nebenwirkungsprofil der jeweiligen Substanzklassen.

\section{Anticholinerge Nebenwirkungen}

Hervorzuheben sind zunächst die bekannten, vorrangig über eine anticholinerge Komponente vermittelten Nebenwirkungen der Trizyklika. Klinisch bedeutungsvoll können werden:

- eine Refluxösophagitis,

- eine verzögerte Darmpassage mit störendem postprandialen Völlegefühl und

- eine schwerwiegende Obstipation, die v. a. bei älteren Patienten bis zum paralytischen Ileus reichen kann.

MAO-Hemmer, Mianserin und Trizyklika mit der Struktur von sekundären Aminen, wie z. B. Desipramin oder Nortriptylin, erscheinen gegenüber solchen, die tertiäre Amine darstellen, durch die geringe oder fehlende anticholinerge Wirkung vorteilhaft. Dies gilt auch für die SSRI, Venlafaxin, Mirtazapin, Reboxetin und Nefazodon.

$\mathrm{Zu}$ beachten sind allerdings die häufigen Klagen über Übelkeit und Erbrechen unter den SSRI, aber auch unter Clomipramin. Sie bilden bei diesen Substanzen die Hauptgründe für ein Absetzen bzw. eine Umstellung auf ein geringer serotonerg wirksames Antidepressivum.

\section{Erkrankungen des Magens und Duodenums}

Trimipramin und Doxepin reduzieren aufgrund ihrer ausgeprägten antihistaminergen Wirkkomponente die 
Säureproduktion des Magens und beschleunigen somit die Heilung des peptischen Ulcus ventriculi und des Ulcus duodeni (Haggerty u. Drossman 1985; Ries et al. 1984).

Cimetidin kann über eine Hemmung des hepatischen oxydativen Metabolismus einen relevanten Anstieg der Antidepressivaplasmaspiegel bewirken. Neuere $\mathrm{H}_{2}$-Antagonisten wie Ranitidin, Famotidin oder Nizatidin erweisen sich aufgrund weniger stark ausgeprägter Medikamenteninteraktionen als vorteilhaft (Epstein 1994).

Erkrankungen im Duodenum können zu einer verminderten Resorption der Antidepressiva führen. In dieser Situation sind Plasmaspiegelmessungen hilfreich und liefern eine rationale Grundlage für eine Dosiskorrektur (Leipzig 1990).

\section{Colon irritabile und entzündliche Darmerkrankungen}

Steht bei einem Colon irritabile die Diarrhö im Vordergrund, ist der Einsatz von anticholinerg wirksamen Trizyklika günstig. Werden wegen Bauchkrämpfen Spasmolytika verordnet, muß bedacht werden, daß auch diese Medikamente eine anticholinerge Wirkung aufweisen und daß additive Effekte möglich sind. Ist die Obstipation das führende Syndrom, kann die zusätzliche Hemmung der Darmmotilität ein toxisches Megakolon induzieren (Drossman u. Thompson 1992).

Vergleichbares gilt für Patienten mit entzündlichen Darmerkrankungen wie M. Crohn und Colitis ulcerosa. Bei der erstgenannten Erkrankung scheint häufiger eine psychiatrische Komorbidität in Form von Angst und Depression vorzuliegen. In jedem Fall muß die potentiell depressionsauslösende Wirkung der Kortikosteroide berücksichtigt werden (Tarter et al. 1987).

\section{Hepatische Erkrankungen, speziell Leberinsuffizienz}

Depressive Störungen bei schweren Lebererkrankungen sind häufig (Trzepacz et al. 1989). Da fast alle Antidepressiva hauptsächlich in der Leber verstoffwechselt werden, sind bei einer Störung der Organfunktion Änderungen der Metabolisierung zu erwarten. Antidepressiva zählen zu den Pharmaka mit einer hohen hepatischen Extraktionsrate, d.h. sie weisen einen bedeutsamen "first-pass"-Effekt auf und unterliegen einer raschen hepatischen Metabolisierung. Der geschwindigkeitsbegrenzende Faktor liegt im Ausmaß des hepatischen Blutflusses. Es sind 2 metabolische Reaktionstypen zu unterscheiden, die bei bestimmten Lebererkrankungen unterschiedlich stark betroffen sein können (Kapfhammer 1993 b; Leipzig 1990; Secor u. Schenker 1987):

Phase-I-Reaktionen. Die Mehrzahl dieser nichtsynthetischen Reaktionen durch Oxydation (Hydroxylierung, N-Demethylierung, Sulfoxidation) findet im glat- ten endoplasmatischen Retikulum (ER) statt. Beteiligt ist das Zytochrom-P-450-System, welches vorrangig perizentral, d.h. um die Zentralvene herum, lokalisiert ist. Weitere nichtsynthetische Reaktionen in Form von Reduktion (Reduktasen) und Hydrolyse (Hydrolasen) laufen hingegen v. a. nicht ER-gebunden ab. Durch Phase-IReaktionen entstehen aktive und inaktive Metaboliten mit einer höheren Wasserlöslichkeit.

Phase-II-Reaktionen. Bei diesen synthetischen Reaktionen, die in der Regel mit einer Inaktivierung einhergehen, werden die in Phase I entstandenen Metabolite entweder durch Bindung an endogene hydrophile Moleküle oder durch Neubildung von - $\mathrm{OH}-,-\mathrm{COOH}-$, $-\mathrm{NH}_{2}$, oder -SH-Gruppen höhergradig polarisiert. In dieser Form können sie leichter von der Niere ausgeschieden werden. Die häufigste Reaktion ist die Glukuronidierung im rauhen ER über die UDPG-Transferase, die v. a. periportal hoch konzentriert ist.

\section{Pharmakodynamik}

Die folgenden pharmakologischen bzw. -kinetischen Schritte bei der Aufnahme und Verstoffwechslung von Antidepressiva können durch verschiedene Lebererkrankungen in unterschiedlichem Ausmaß betroffen sein (Sellers u. Bendyan 1987):

Intestinale Absorption. Zirrhotischer Umbau der Leber und portaler Hypertonus können zu einer bedeutsamen Verzögerung der Absorption führen.

Metabolisierung. Akute virale Hepatitiden und alkoholische Hepatopathien betreffen bevorzugt die perizentrale Region. Sie beeinträchtigen als Konsequenz in erster Linie die oxydativen Enzymsysteme. Bei chronischen Hepatitiden hingegen wird v.a. die periportale Region in Mitleidenschaft gezogen. Solange noch kein zirrhotischer Umbau stattgefunden hat, bleiben also die oxydativen nichtsynthetischen Reaktionen lange Zeit weitgehend unberührt. Bei einer primären biliären Zirrhose verhält es sich ganz analog. Die Glukuronidierungsschritte können infolge eines besonderen Schutzes hinter einer lipophilen „Barriere“ sowie extrahepatischer Ausweichmöglichkeiten im Darm und in der Niere sowohl bei akuten als auch bei chronischen Hepatitiden relativ intakt bleiben. Azetylierungsreaktionen, die ähnlich wie die Phase-I-Reaktionen v. a. perizentral lokalisiert sind, werden sowohl bei akuten als auch chronischen Lebererkrankungen stark reduziert.

Plasmaproteinbindung. Eine eingeschränkte Syntheseleistung führt zu einer reduzierten Albuminkonzentration im Serum. Reziprok steigt der Anteil der freien, nichtgebundenen Pharmkakonzentration an. Analoge Effekte können beobachtet werden, wenn vermehrt anfallende endogene Hemmsubstanzen eine Ver- 
drängung der aktiven Wirkmoleküle einzelner Pharmaka aus ihrer Bindung an Albumin verursachen.

Verteilungsvolumen. Aszites und Ödembildung können zu einer Vergrößerung des Verteilungsvolumens eines bestimmten Pharmakons führen.

Elimination. Extrahepatische Shuntbildungen, aber auch eine Obliteration der sinusoidalen Architektur reduzieren die hepatischen Eliminationsleistungen. Aus diesem Grund ist bei Vorliegen einer Zirrhose der oben beschriebene First-pass-Effekt erheblich vermindert.

\section{Metabolisierung und aktive Metabolite}

Die hepatische Metabolisierung der tri- und tetrazyklischen Antidepressiva, aber auch von MAO-Hemmern, SSRI, Venlafaxin, Mirtazapin, Reboxetin und Nefazoden geschieht v. a. über das perizentral lokalisierte oxydative Zytochrom-P-450-System. Es entstehen hierüber aktive Metabolite mit einer relativ langen Halbwertszeit. Diese Phase-I-Reaktionen können besonders bei akuten viralen Hepatitiden und alkoholtoxisch bedingten Hepatopathien empfindlich gestört werden.

Bei trizyklischen Antidepressiva entstehen unter diesen Umständen aktive Metaboliten, deren Halbwertszeit auf ein Vielfaches derjenigen unter ungestörten Bedingungen verlängert sein kann. Ein wichtiger Hinweis auf eine solche Situation kann eine ausgeprägte Sedierung sein. Ein verminderter First-pass-Effekt macht es notwendig, nicht nur die Erhaltungsdosis $\mathrm{zu}$ reduzieren, sondern auch schon die Initialdosis.

Bei Patienten mit einer Verlangsamung des intestinalen Transits kann die anticholinerg bedingte zusätzliche Verweildauer des Darminhalts zu einer verstärkten Absorption von toxischen Substanzen führen und so eine hepatische Enzephalopathie anstoßen.

Das Risiko der Triggerung einer hepatischen Enzephalopathie bzw. der Demaskierung subklinischer Formen besteht auch bei MAO-Hemmern.

\section{Einzelne Präparate}

- Fluoxetin. Fluoxetin unterscheidet sich gegenüber den anderen SSRI durch eine wesentlich längere Halbwertszeit. Sie beträgt 2-3 Tage gegenüber einer Halbwertszeit von ca. $24 \mathrm{~h}$ bei Paroxetin oder Sertralin. Sein aktiver Hauptmetabolit Norfluoxetin besitzt sogar eine Halbwertszeit von 7-9 Tagen. Bei einer Leberzirrhose verlängern sich diese Halbwertszeiten von Fluoxetin und Norfluoxetin auf je ca. 8 bzw. 12 Tage. Bei Vorliegen einer Leberdekompensation können diese Halbwertszeiten noch zusätzlich ansteigen.

Um keine toxischen Effekte auszulösen, müssen die Tagesdosen daher deutlich reduziert werden. Um den veränderten pharmakologischen und -kinetischen Bedingungen in erster Annäherung gerecht zu werden, sollte die Dosis dieser Pharmaka um 50\% reduziert werden. Die Gabe an jedem zweiten Tag stellt ein prak- tikables Alternativverfahren dar (Castiella u. Arenas 1994; Schenker et al. 1988). Zahlreiche Interaktionen mit anderen Medikamenten infolge einer ausgeprägten Hemmung des oxydativen Zytochrom-P-450-Systems machen den Einsatz von Fluoxetin beim Patienten mit schwerer Lebererkrankung zusätzlich problematisch (Ciraulo u. Shader 1990 a, b).

Paroxetin, Sertralin und Fluvoxamin. Auch Paroxetin sollte bei Leberpatienten in einer reduzierten Dosis verabreicht werden. Es interagiert in einer ähnlichen Weise wie Fluoxetin mit dem Zytochrom-P-450-System. Sertralin bildet in dieser Hinsicht eine bemerkenswerte Ausnahme, da es nur eine minimale Interaktion mit diesem Oxydationssystem aufweist. Fluvoxamin hat eine Halbwertszeit von ca. $15 \mathrm{~h}$, die bei Vorliegen einer Leberzirrhose auf ca. $24 \mathrm{~h}$ ansteigen kann. Im Unterschied zu anderen SSRI inhibiert es jedoch das ZytochromP450-1 A 2 -Subsystem, dessen Substrat u.a. auch Clozapin ist (Brosen 1996; Goodnick 1994).

Venlafaxin. Venlafaxin (HWZ: 4,1 \pm 1,3 h, Desmethyl-Venlafaxin: HWZ: $10,4 \pm 1,7 \mathrm{~h}$ ) interagiert mit anderen Medikamenten, die das Zytochrom-P-450-2D Subsystem hemmen (s. oben). Da bei Bestehen einer Leberzirrhose die Halbwertszeit von Venlafaxin um ca. ein Drittel, die seines aktiven Hauptmetaboliten um ca. $60 \%$ ansteigt, sollten die sonst üblichen Dosierungen um etwa $50 \%$ reduziert werden (Holliday u. Benfield 1995).

Mirtazapin. Mirtazapin zeigt eine Eliminationshalbwertszeit von 20-40 h. In geringerem Ausmaß wird auch ein aktiver Metabolit Demethyl-Mirtazapin gebildet. Bei Leberfunktionsstörungen kann es zu einem Anstieg der Halbwertszeit um ca. $40 \%$ kommen. Eine Dosisreduktion ist deshalb bei dieser Patientengruppe ebenfalls empfehlenswert (Montgomery 1995).

Trazodon. Entgegen des früher unter dieser Indikationssstellung als potentiell vorteilhafter eingestuften Trazodons (Secor u. Schenker 1987), haben neuere pharmakologische Untersuchungen auch für Trazodon eine intensive hepatische Metabolisierung nachgewiesen (Rotzinger et al. 1999). Fälle einer Trazodon-induzierten Hepatotoxizität wurden berichtet (Fernandes et al.2000). Dies gilt auch für das strukturchemisch sehr verwandte neuere Antidepressivum Nefazodon (Ehrentraut et al. 2002). Es ist also eine große Zurückhaltung bei depressiven Patienten mit bereits bekannten Leberschädigungen angesagt.

\section{Dosierung}

Die bei einer Leberinsuffizienz notwendige Dosisreduktion der meisten Antidepressiva beträgt in aller Regel zwischen 30 und $50 \%$. Führen im weiteren Krankheitsverlauf eingeschränkte Syntheseleistungen zu reduzierten Albuminkonzentrationen, können reziprok die 
Fraktionen der freien, ungebundenen Wirkkonzentrationen der Antidepressiva ansteigen. Aszites und Ödembildung wiederum vergrößern das Verteilungsvolumen. In beiden Fällen ist eine sorgfältige Kontrolle der Antidepressivaplasmakonzentrationen anzuraten (Kapfhammer 1993b).

\section{Nierenerkrankungen}

Patienten mit chronischen Nierenerkrankungen und konsekutiver Dialysepflichtigkeit sind in hohem Maße anfällig für depressive Störungen (Surman 1991). Die chronische Erkrankung betrifft fast alle psychosozialen Lebensbereiche und beeinträchtigt Körperbild, Sexualität, Familienleben, Arbeitsfähigkeit usw. Urämische Symptome, Anämie, Elektrolytstörungen, aber auch die zugrunde liegende organische Erkrankung, wie beispielsweise ein Lupus erythematodes, können aufgrund einer ähnlichen klinischen Symptomatologie einen depressiven Zustand mimikryartig darstellen. Der Internist muß - soweit möglich - eine sorgfältige Korrektur dieser Zustände vornehmen, bevor neben psychotherapeutischen Techniken auch an den Einsatz von Antidepressiva gedacht werden kann.

Da alle Antidepressiva mit Ausnahme von Trazodon bevorzugt hepatisch metabolisiert werden, können sie auch bei Niereninsuffizienz eingesetzt werden. Eine renale Elimination der Muttersubstanzen erfolgt nicht oder nur in geringfügigem Umfang. Dies gilt aber nicht in gleichem Maße für die bei der hepatischen Verstoffwechselung anfallenden unterschiedlichen Metabolite. Diese werden wiederum überwiegend renal, in variablem Prozentsatz auch biliär ausgeschieden.

Patienten unter Dialyse. Da Antidepressiva wie die meisten Psychopharmaka eine hohe Plasmaproteinbindung aufweisen, besteht für sie keine relevante Dialysierbarkeit (Kapfhammer 1993 b).

Bei einer Niereninsuffizienz müssen deshalb Antidepressiva nach durchgeführter Dialyse nicht substituiert werden. Systemische pH-Verschiebungen können aufgrund einer veränderten Verteilung der Wirksubstanzen höhere Plasmakonzentrationen der freien Wirksubstanzen verursachen und so eine Reduktion der Tagesdosen notwendig machen.

Aktive Metabolite. Klinisch ist v. a. das Problem der aktiven, insbesondere der konjugierten Metabolite zu beachten, die u. a. für außerordentlich störende Sedierungseffekte und eine erhöhte Sensitivität gegenüber anderen Nebenwirkungen verantwortlich gemacht werden. Im Hinblick darauf empfiehlt sich eine Dosisreduktion (Dawling et al. 1982; Lieberman et al. 1985; Sellers u. Bendayan 1987).

Trizyklika. Vor allem bei stark anticholinerg wirksamen trizyklischen Antidepressiva kann eine ohnehin meist schon aufgrund der somatischen Bedingungen vorliegende orthostatische Hypotension verstärkt und die verbleibende Harnausscheidung weiter reduziert werden.

Trizyklische Antidepressiva zeigen wichtige Interaktionen mit einer Reihe von internistisch indizierten Medikamenten, die bei niereninsuffizienten Patienten häufig eingesetzt werden. So können Trizyklika die über eine Stimulation zentraler $\alpha_{2}$-Adrenorezeptoren vermittelte Blutdrucksenkung durch Clonidin aufheben. Ähnliche Effekte lassen sich auch bei anderen Antihypertonika wie Guanethidin, Methyldopa oder Reserpin beobachten (Blackwell 1981). Günstiger ist hier der Einsatz von ß-Blockern, Diuretika oder Hydralazin. Mianserin und Maprotilin zeigen diese Interaktionen nicht.

Als eine allgemeine Regel kann gelten, daß trizyklische Antidepressiva bei Patienten mit Nierenerkrankungen, speziell mit Niereninsuffizienz zunächst in niedriger Dosierung einschleichend verabreicht und eine Höherdosierung unter sorgfältiger Kontrolle auftretender Sedierungseffekte, einer orthostatischen Hypotension und anderer Nebenwirkungen vorgenommen werden sollte.

MAO-Hemmer. Die klassischen MAO-Hemmer sind auch bei niereninsuffizienten Patienten mit gutem Erfolg eingesetzt worden. Hier ist aber v. a. die wenig kalkulierbare orthostatische Hypotension als klinisches Problem zu beachten (Levy 1990).

SSRI. Der Einsatz der moderneren Präparate wie z.B. der SSRI ist für die geschilderte Patientengruppe bisher noch wenig erforscht worden. Die Plasmakonzentrationen von Fluoxetin und Norfluoxetin scheinen durch eine Hämodialyse kaum verändert zu werden. Zwischen renaler Funktionseinschränkung und der Eliminationsgeschwindigkeit, dem Verteilungsvolumen oder der Plasmaproteinbindung wurde bei Fluoxetin kein Zusammenhang gefunden (Aronoff et al. 1984; Bergstrom et al. 1993). Andererseits ist Fluoxetin zu einem sehr hohen Prozentsatz (94\%) an Plasmaprotein gebunden und kann eine Reihe von anderen Medikamenten, beispielsweise Digoxin oder Phenprocoumon (Marcumar), aus dieser Proteinbindung verdrängen. Durch den erhöhten freien Anteil dieser Substanzen kann eine gesteigerte klinische Wirkung verursacht werden. Auch bei zahlreichen anderen Medikamenten kann Fluoxetin erhöhte Plasmaspiegelkonzentrationen induzieren. Hierzu zählen Cyclosporine, $\beta$-Blocker, Antikonvulsiva, Antiarrhythmika und v. a. andere Psychopharmaka. Eine vorsichtiges Vorgehen mit niedrigen Dosierungen muß deshalb für Fluoxetin bei nierenkranken Patienten empfohlen werden.

Paroxetin. Auch zum Einsatz von Paroxetin beim niereninsuffizienten Patienten liegen bislang kaum em- 
pirische Daten vor. Steigende Plasmaspiegel in Abhängigkeit vom Grad der renalen Funktionsstörung legen insgesamt niedrigere Tagesdosen nahe (Doyle et al. 1989).

Venlafaxin. Die Clearance von Venlafaxin und seinem Hauptmetaboliten ist bei renalen Erkrankungen signifikant erniedrigt. Eine Dosisanpassung ist deshalb notwendig (Troy et al. 1994).

\section{Karzinome}

Vor dem Beginn einer jeden antidepressiven Medikation bei Karzinomleiden sollten somatische Faktoren oder Medikamente mit spezifischer Wirkung als Auslöser der depressiven Störung erwogen werden. So ist speziell an

- Hirnmetastasen,

- Elektrolytverschiebungen,

- Ernährungsmängel,

- Nebenwirkungen einer Chemotherapie oder einer Radiatio

$\mathrm{zu}$ denken. Unter den onkologisch eingesetzten Pharmaka zeichnen sich Vincristin, Vinblastin, L-Asparaginase, Dacarbazin, Procarbazin, Hexamethylamin, aber auch Interferon und v. a. Kortikosteroide durch einen bedeutsamen depressionsauslösenden Effekt aus (Kapfhammer 1993 a).

Der Einsatz irreversibler MAO-Hemmer bei Tumorpatienten wird allgemein zurückhaltend beurteilt. Moclobemid könnte aber durchaus eine Anwendungsmöglichkeit behaupten. Die SSRI, aber auch Venlafaxin oder Mirtazapin, versprechen unter dieser Indikationsstellung eine vorteilhafte Anwendung. Das Problem der häufigen Nebenwirkungen von Übelkeit und Erbrechen ist aber gesondert zu beachten. Psychostimulanzien werden dann als wichtige Therapiealternative erwogen, wenn ein rascher Effekt bei anergen Zuständen wünschenswert ist. Eine gute analgetische Zusatzwirkung sowie eine fehlende Sedierung können sich als Vorteile erweisen.

Nebenwirkungen und Interaktionen. Der Einsatz eines bestimmten Antidepressivums kann sich an den Zielsymptomen des depressiven Syndroms orientieren (Bluestine u. Lesko 1994). Entsprechend ihres Nebenwirkumsspektrums bewähren sich Amitriptylin oder Doxepin bei agitierten Patienten mit ausgeprägten Schlafstörungen, Nortriptylin oder Desipramin eher bei Zuständen, bei denen eine starke Sedierung unerwünscht ist. Antidepressiva mit deutlich anticholinerger Wirkung sollten vermieden werden, wenn eine chemooder radiotherapeutisch bedingte Stomatitis, eine verringerte Darmmotilität oder eine Blasenentleerungsstörung, beispielsweise nach einem operativen Eingriff, vorliegen. Metoclopramid, welches häufig wegen seiner antiemetischen Wirkung eingesetzt wird, kann zu einer ausgeprägten Plasmaspiegelerhöhung der trizyklischen Antidepressiva führen.

Darreichung und Dosierung. Von klinischer Bedeutsamkeit kann sein, daß die meisten trizyklischen Antidepressiva auch als Suppositorien aufbereitbar sind. Amitriptylin, Imipramin und Doxepin lassen sich auch i. m. applizieren. Eine häufig veränderte Stoffwechsellage, meist zahlreiche Medikamenteninteraktionen sowie eine möglicherweise verstärkte Nebenwirkungssensibilität erfordern in aller Regel eine antidepressive Medikation in niedrigeren Dosierungen.

Schmerztherapie. Bei Patienten ohne depressive Symptomatik sollten Antidepressiva nicht als Monotherapie zur Bekämpfung von Schmerzzuständen eingesetzt werden. Obgleich viele dieser Substanzen einen eigenständigen analgetischen Effekt besitzen, stellen sie doch nur ein Adjuvans in der Schmerzbehandlung dar. Allerdings ist es durch ihre Anwendung möglich, andere Analgetika, beispielsweise Morphinpräparate, einsparen.

\section{Patienten unter Lithiumprophylaxe}

Patienten, die wegen einer phasenhaft verlaufenden affektiven Erkrankung prophylaktisch Lithium einnehmen, stellen einen Sonderfall innerhalb des medizinischen Behandlungssettings dar, auch wenn sie keine depressive Verstimmung aufweisen (Müller et al. 1995). $\mathrm{Zu}$ berücksichtigen sind einerseits Arzneimittelinteraktionen, andererseits der Einfluß der Erkrankung selbst auf den Lithiumspiegel.

Interaktionen. Thiazide, Spironolacton und Triamteren, Kalziumkanalblocker, ACE-Hemmer sowie nichtsteroidale Antiphlogistika bewirken eine signifikante Lithiumspiegelerhöhung und können zu einer Lithiumintoxikation führen. Metronidazol erhöht ebenfalls den Lithiumspiegel und verändert das Risiko einer Nephrotoxizität unter Lithium. Theophylline, Aminophylline und Azetazolamid erniedrigen wiederum den Lithiumspiegel und können den prophylaktischen Schutz klinisch relevant absenken (Stoudemire et al. 1991).

Einfluß der Erkrankung selbst. Bei zahlreichen internistischen Erkrankungen können Begleitumstände wie Erbrechen, Durchfälle, Polyurie oder starkes Schwitzen zu einer bedeutsamen Verringerung des Körperflüssigkeitsvolumens führen und hierüber einen u. U. bedenklichen Anstieg des Lithiumspiegels bewirken. Dieser Effekt kann zusätzlich verstärkt werden, wenn bereits vorher eine lithiuminduzierte Polyurie bestand. 


\section{Operationen}

Das Problem des Flüssigkeitsvolumens akzentuiert sich, wenn sich der Patient einer Operation unterziehen muß und eine präoperativ angeordnete Nahrungs- und Flüssigkeitskarenz den Konzentrationsvorgang noch weiter intensiviert. Für eine ausreichende parenterale Flüssigkeitssubstitution muß also gesorgt werden. 2-3 Tage vor dem elektiven Operationstermin sollte Lithium abgesetzt werden. Zwischen Lithium und Narkosemittel bestehen keine Nebenwirkungen. Eine mögliche Wirkverlängerung von Muskelrelaxantien ist aber zu bedenken. Sobald postoperativ eine ausgeglichene Flüssigkeitsbilanz vorliegt, spätestens aber wenn der Patient wieder selbständig trinkt, ist Lithium erneut anzusetzen.

Eine Lithiumpause von wenigen Tagen bedeutet für den Patienten im Regelfall keine signifikante Minderung seines Rezidivschutzes. Einige wenige Patienten aber, bei denen aus früheren Absetzversuchen eine rasche psychopathologische Destabilisierung bekannt ist, benötigen während dieser lithiumfreien Zeit eine besonders engmaschige Überwachung. Ein überbrückender Einsatz eines Neuroleptikums oder eines Benzodiazepins kann notwendig sein.

\section{Nierenerkrankungen}

Bei Patienten mit akutem Nierenversagen ist die Fortführung der Lithiummedikation kontraindiziert. Ein Ausweichen auf Neuroleptika ist möglich. Patienten mit chronischer Niereninsuffizienz und Dialysepflichtigkeit sind bei sorgfältiger Überwachung aber in aller Regel ohne größere Probleme mit Lithium weiterzubehandeln. Lithium ist voll dialysabel. Nach einer Dialyseeinheit genügt die Substitution durch eine Einmalgabe von ca. 300-600 mg, da bis zum nächsten Dialysetermin die Spiegel aufrecht erhalten bleiben. Initial empfiehlt sich eine Konzentrationsbestimmung jeweils nach der Postdialysegabe. Später sollte aber auch unmittelbar vor einer Dialyseeinheit eine Messung durchgeführt werden, um ein zwischenzeitliches Ansteigen der Konzentration erfassen und korrigieren zu können.

Patienten nach einer Nierentransplantation können in der Akutphase wegen der massiven perioperativen Wasser- und Elektrolytverschiebungen nicht mit Lithium behandelt werden. Dies wird in einem stabilen postoperativen Abschnitt aber in aller Regel wieder möglich. Da nierentransplantierte Patienten meist Kortikosteroide und/oder Cyclosporine einnehmen, müssen deren vermindernde Effekte auf die Lithiumclearence berücksichtigt werden. Eine Dosisanpassung durch reduzierte Lithiumgabe ist vorzunehmen (Kapfhammer 1993b).

\section{Literatur}

Ammon Cavanaugh S von (1984) Diagnosing depression in the hospitalized patient with chronic medical illness. J Clin Psychiatry $45: 13-16$

Ammon Cavanaugh S von (1995) Depression in the medically ill. Critical issues in diagnostic assessment. Psychosomatics 36: $48-59$

Ananth J (1974) Antiasthmatic effect of amitriptyline. Can Med Assoc J 110:1133

Ananth J, Swartz R, Burgoyne K et al. (1994) Hepatic disease and psychiatric illness. Psychother Psychosom 62:146-159

Andersen J, Aabro E, Gulman N (1980) Antidepressant treatment in parkinson's disease. Acta Neurol Scand 62:210-219

Aronoff G, Bergstrom R, Pottratz S et al. (1984) Fluoxetine kinetics and protein binding in normal and impaired renal function. Clin Pharmacol Ther 36:138-144

Baldessarini RJ (1996) Drugs and the treatment of psychiatric disorders. Depression and mania. In: Hardman JG, Limbird LE (eds) Goodman \& Gilman's The Pharmacological Basis of Therapeutics, 9th edn. McGraw-Hill, New York, p 431

Bandelow B, Rüther E (1993) Besonderheiten der Psychopharmakatherapie bei psychisch Kranken mit körperlichen Erkrankungen. In: Möller HJ (Hrsg) Therapie psychiatrischer Erkrankungen. Enke, Stuttgart, S 791-800

Bass S, Colebatch H (1992) Fluoxetine-induced lung damage (letter). Med J Aust 156:364-365

Beckson M, Cummings JL (1992) Neuropsychiatric aspects of stroke. Int J Psychiatry Med 21:1-15

Beitman BD, Mukerji J, Lamberti JW et al. (1989) Panic disorder in patients with chest pain and angiographically normal coronary arteries. Am J Cardiol 63:1399-1403

Bergstrom R, Beasley C, Levy N et al. (1993) The effect of renal and hepatic disease on the pharmacokinetics, renal tolerance, and risk-benefit profile of fluoxetine. Int Clin Psychopharmacol 8: 261-266

Berrios GE, Quemada JI (1990) Depressive illness in multiple sclerosis. Br J Psychiatry 156:10-16

Beutel M, Weiner H (1993) Trauer und Depression nach einem Objektverlust. Forum Psychoanal 9:224-239

Bigger JT, Fleiss JL, Steinman RC et al. (1992) Frequency domain measures of heart rate period variability and mortality after myocardial infarction. Circulation 85:164-171

Bishay NR, Tarrier N, Roberts AP (1995) Cognitive therapy of agoraphobia in reaction to physical illness: An uncontrolled study. Ir J Psychol Med 12:135-138

Blackwell B (1981) Adverse effects of antidepressant drugs. Drugs $21: 201-219$

Bluestine S, Lesko L (1994) Psychotropic medications in oncology and in AIDS patients. Adv Psychosom Med 21:107-137

Borson S, McDonald G, Gayle T et al. (1992) Improvement in mood, physical symptoms, and functions with nortriptyline for depression in patients with chronic obstructive pulmonary disease. Psychosomatics 33:190-201

Bouckoms A, Hackett TP (1991) The pain patient: Evaluation and treatment. In: Cassem N (ed) Massachusetts General Hospital Handbook of General Hospital Psychiatry, 3rd edn. Mosby Year Book, St. Louis, pp 39-68

Brosen K (1996) Are pharmacokinetic drug interactions with the SSRI an issue? Int Clin Psychopharmacol 11 (Suppl 1): 23-27

Brown JH, Paraskevas F (1982) Cancer and depression: Cancer presenting with depressive illness: An autoimmune disease? $\mathrm{Br} \mathrm{J}$ Psychiatry $141: 227-232$

Brown RG, MacCarthy B, Gotham AM et al. (1988) Depression and disability in Parkinson's disease. Psychol Med 18:49-55

Brown TM, Stoudemire A, Fogel BS, Moran MG (2000) Psychopharmacology in the medical patient. In: Stoudemire A, Fogel BS, Greenberg DB (eds) Psychiatric care of the medical patient. $2^{\text {nd }}$ edn. Oxford University Press, Oxford, pp 329-372

Bukberg J, Penman D, Holland JS (1984) Depression in hospitalized cancer patients. Psychosom Med 46:199-212

Burvill PW, Johnson GA, Vamrozik KD et al. (1995) Anxiety disorders after stroke: Results from the Perth Community Stroke Study. Br J Psychiatry 166:328-332 
Callies AL, Popkin MK (1987) Antidepressant treatment in medical-surgical inpatients by nonpsychiatric physicians. Arch Gen Psychiatry 44:157-160

Cassem EH (1988) Depression secondary to medical illness. Rev Psychiatry 7:256-273

Cassem EH (1990) Depression and anxiety secondary to medical illness. Psychiatr Clin North Am 13:597-612

Cassem EH (1995) Depressive disorders in the medically ill. Psychosomatics 36:2-10

Castiella A, Arenas J (1994) Fluoxetine hepatotoxicity (letter). Am J Gastroenterol 89:458-459

Chiarello RJ, Cole JO (1987) The use of psychostimulants in general psychiatry: A reconsideration. Arch Gen Psychiatry 44: 286-295

Chochinov HM, Wilson KG, Enns M et al. (1994) Prevalence of depression in the terminally ill: Effects of diagnostic criteria and symptom threshold judgements. Am J Psychiatry 4:537-540

Ciraulo DA, Shader RI (1990 a) Fluoxetine drug-drug interactions, I. Antidepressants and antipsychotics. J Clin Psychopharmacol $10: 48-50$

Ciraulo DA, Shader RI (1990 b) Fluoxetine drug-drug interactions, II. J Clin Psychopharmacol 10:213-217

Clark DC, von Ammon Cavanaugh S, Gibbons RD (1983) The core symptoms of depression in medical and psychiatric patients. J Nerv Ment Dis 171:705-713

Clark DA, Cook A, Snow D (1998) Depressive symptom differences in hospitalized, medically ill, depressed psychiatric inpatients and nonmedical controls. J Abnorm Psychol 107:38-48

Clayton PJ, Lewis CE (1981) The significance of secondary depression. J Affect Disord 3:25-35

Cohen-Cole SA, Stoudemire A (1987) Major depression and physical illness. Special considerations in diagnosis and biologic treatment. Psychiatr Clin North Am 10:1-17

Colon EA, Popkin MK (1996) Anxiety and panic. In: Randell JR, Wise MG (eds) Textbook of consultation-liaison psychiatry. American Psychiatric Press, Washington/DC, pp 402-425

Costa D, Mogos I, Toma T (1985) Efficacy and safety of mianserin in the treatment of depression of women with cancer. Acta Psychiatr Scand 72 (Suppl 329): 85-92

Coulehan JI, Schulberg HC, Block MR, Zettler-Segal M (1988) Symptom patterns of depression in ambulatory medical and psychiatric patients. J Nerv Ment Dis 176:284-288

Craven JL, Rodin GM, Johnson L, Kennedy SH (1987) The diagnosis of major depression in renal dialysis patients. Psychosom Med 49:482-492

Creed F (1997) Assessing depression in the context of physical illness. In: Robertson MM, Katona CLE (eds) Depression and physical illness. Wiley \& Sons, Chichester, pp 3-19

Cull A (1990) Invited review: Psychological aspects of cancer and chemotherapy. J Psychosom Res 34:129-140

Cummings JL (1992) Depression and Parkinson's disease. Am J Psychiatry 149:443-454

Dalack GW, Roose SP (1990) Perspectives on the relationship between cardiovascular disease and affective disorder. J Clin Psychiatry 51 (Suppl): 4-9

Dawling S, Lynn K, Rosser R et al. (1982) Nortriptyline metabolism in chronic renal failure: Metabolite elimination. Clin Pharmacol Ther 32:322-329

De Vito AJ (1990) Dyspnoe during hospitalizations for acute phase of illness as recalled by patients with chronic obstructive airway disease. Heart Lung 19:186-191

Derogatis LR, Wise TN (eds) (1989) Anxiety and depressive disorders in the medical patient. American Psychiatric Press, Washington, DC

Derogatis LR, Morrow GR, Fetting J et al. (1983) The prevalence of psychiatric disorders among cancer patients. JAMA 249: 751-757

Deshpande SN, Sundaram KR, Wig NN (1989) Psychiatric disorders among medical inpatients in an Indian hospital. Br J Psychiatry 154:504-509

Dew MA, Ragi MV, Nimorwicz P (1990) Infection with human immunodeficiency virus and vulnerability to psychiatric distress. Arch Gen Psychiatry 47:737-744

Dietch J (1981) Diagnosis of organic anxiety. Psychosomatics 22: 661-665
Dietch J, Fine M (1990) The effect of nortriptyline in elderly patients with cardiac conduction disease. J Clin Psychiatry 51: 65-67

Doyle G, Laher M, Kelly J et al. (1989) The pharmacokinetics of paroxetine in renal impairment. Acta Psychiatr Scand 80 (Suppl 350): 89-90

Drossman DA, Thompson WG (1992) The irritable bowel syndrome: Review and a graduated multicomponent treatment approach. Ann Intern Med 116:1009-1016

Ehrentraut S, Rothenhäusler HB, Gerber A, Rau HG, Thiel M, Schirren CA (2000) Akutes Leberversagen unter NefazodonTherapie? Nervenarzt 73:686-689

Eilenberg MD (1960) Psychiatric illness and pernicious anemia J Ment Sci 106:1539-1548

Epstein SA (1994) Psychotropic medications in gastrointestinal and hepatic disease. In: Silver P (ed) Psychotropic drug use in the medically ill. Adv Psychosom Med, vol 21. Karger, Basel, pp 49-60

Ereshefsky L (1996) Drug interactions of antidepressants. Psychiatr Ann 26:342-350

Evans M, Hammond M, Wilson K et al. (1997) Placebo-controlled treamtent trial of depression in elderly physically ill patients. Int J Geriatr Psychiatry 12:817-824

Fava GA (1994) Affective disorders and endocrine disease. New insights from psychosomatic studies. Psychosomatics 35: $341-353$

Fava GA, Molnar G (1987) Criteria for diagnosing depression in the setting of medical disesase. Psychother Psychosom 48:21-25

Fava GA, Sonino N (1996) Depression associated with medical illness. CNS Drugs 5:175-189

Fava GA, Sonino N, Morphy MA (1993) Psychosomatic view of endocrine disorders. Psychother Psychosom 59:20-33

Fava GA, Trombini G, Barbara L et al. (1985) Depression and gastrointestinal illness: The joint use of biological and clinical criteria. Am J Gastroenterol. 80:195-199

Fava GA, Sonino N, Wise TN (1988) Management of depression in medical patients. Psychother Psychosom 49:81-102

Fava M, Rosenbaum JF, Pava J et al. (1993) Anger attacks in unipolar depression, part 1: Clinical correlates and response to fluoxetine treatment. Am J Psychiatry 150:1153-1163

Fawzy FI, Fawzy NW, Hyun CS et al. (1993) Malignant melanome: Effects of an early structured psychiatric intervention, coping, and affective state on recurrence and survival 6 years later. Arch Gen Psychiatry 50:681-689

Fawzy FI, Fawzy NW, Canada AL (1998) Psychosocial treatment of cancer: An update. Curr Opin Psychiatry 11:601-605

Feldman E, Mayou R, Hawton K et al. (1987) Psychiatric disorder in medical inpatients. QJM 63:405-412

Fernandes NF, Martin RR, Schenker S (2000) Trazodone-induced hepatotoxicity. A case report with comment on drug-induced hepatotoxicity. Am J Gastroenterol 95:532-538

Fifer SK, Mathias SD, Patrick DL et al. (1994) Untreated anxiety among adult primary care patients in a health maintenance organization. Arch Gen Psychiatry 51:740-750

Fogel BS (1990) Major depression versus organic mood disorder: A questionable distinction. J Clin Psychiatry 51:53-56

Folstein SE, Abbott MH, Chase GA et al. (1983) Huntington's disease in a case series and in a family. Psychol Med 13:537-542

Ford MF (1986) Treatment of depression in Huntington's disease with monoamine oxidase inhibitors. Br J Psychiatry 149: 654-656

Frank RG, Beck NC, Parker JC et al. (1988) Depression in rheumatoid arthritis. J Rheumatol 15:920-925

Frasure-Smith N (1991) In-hospital symptoms of psychological stress as predictors of long-term outcome after acute myocardial infarction in men. Am J Cardiol 67:121-127

Frasure-Smith N, Lesperance F, Talajic M (1993) Depression following myocardial infarction, impact on 6-month survival. JAMA 270:1819-1825

Freedland KE, Carney RM, Lustman PJ et al. (1992) Major depression in coronary artery disease patients with vs. without prior history of depression. Psychosom Med 54:416-421

Gellert GA, Maxwell RM, Siegel BS (1993) Survival of breast cancer patients receiving adjunctive psychosocial support therapy: A 10-year follow-up study. J Clin Oncol 11:66-69 
Goldberg RJ, Posner DA (1993) Anxiety in the medically ill. In: Stoudemire A, Fogel BS (eds) Psychiatric care of the medical patient. Oxford University Press, New York, pp 87-104

Gonzalez-Rothi R, Zander D, Ros P (1995) Fluoxetine hydrochloride (Prozac)-induced pulmonary disease. Chest 107:1763-1765

Goodnick PJ (1994) The pharmacokinetic optimization of treatment with newer antidepressants. Clin Pharmacokinet 27: 307-330

Gore-Felton C, Spiegel D (2000) Group psychotherapy for medically ill patients. In: Stoudemire A, Fogel BS, Greenberg DB (eds) Psychiatric care of the medical patient. $2^{\text {nd }}$ edn. Oxford University Press, Oxford, pp 41-49

Greer S (1994) Psycho-oncology: Its aims, achievements, and future tasks. Psychooncology 3:87-101

Guthrie E (1996) Emotional disorders in chronic illness: Psychotherapeutic interventions. Br J Psychiatry 168:265-273

Haggerty JJ, Drossman DA (1985) Use of psychotropic drugs in patients with peptic ulcer. Psychosomatics 26:277-284

Hama N, Boumpas DTD (1995) Cerebral lupus erythematosus. CNS Drugs 3:416-426

Harrison J, Maguire P (1994) Predictors of psychiatric morbidity in cancer patients. Br J Psychiatry 165:593-598

Hayward C, Clark DB, Taylor CB et al. (1990) Panic disorder, anxiety, and cardiovascular risk. In: Ballenger JC (ed) Clinical aspects of panic disorder. Wiley-Liss, New York, pp 99-110

Henry JA (1989) A fatal toxicity index for antidepressant poisoning. Acta Psychiatr Scand 80:37-45

Hinrichsen GA, Liebermann JA, Pollack S et al. (1989) Depression in hemodialysis patients. Psychosomatics 30:284-289

Holland JC, Hughes A, Korzan AH et al. (1986) Comparative psychological disturbance in patients with pancreatic and gastric cancer. Am J Psychiatry 143:982-986

Holliday SM, Benfield P (1995) Venlafaxine - A review of its pharmacology and therapeutic potential in depression. Drugs 49: 280-294

Horowitz MJ (1976) Stress response syndromes. Jason Aronson, New York

Horowitz MJ, Siegel B, Holen A et al. (1997) Diagnostic criteria for complicated grief disorder. Am J Psychiatry 154:904-910

Hughes JE (1985) Depressive illness and lung cancer. Eur J Surg Oncol 11:15-20

Judd FK, Mijch AM (1994) Depression in patients with HIV and AIDS. Aust NZ. J Psychiatry $28: 642-650$

Kaasa S, Malt U, Jagen S et al. (1993) Psychological distress in cancer patients with advanced disease. Radiotherp Oncol 27 192-197

Kapfhammer HP (1993a) Epidemiologie der Depression im Rahmen von Tumorerkrankungen. In: Staab HJ, Ludwig M (Hrsg) Depression bei Tumorpatienten. Thieme, Stuttgart, S 29-41

Kapfhammer HP (1993b) Nieren- und leberinsuffiziente Patienten - ein therapeutisches Problem. In: Möller HJ, Przuntek H (Hrsg) Therapie im Grenzgebiet von Psychiatrie und Neurologie. Springer, Berlin Heidelberg New York Tokyo, S 75-92

Kapfhammer HP (1995) Benzodiazepine bei Angsterkrankungen. In: Kasper S, Möller HJ (Hrsg) Praxis der Angst- und Panikerkrankungen. Fischer, Stuttgart, S 261-288

Kapfhammer HP (1996) Depression und Suizidalität bei Krebserkrankungen. TW Neurologie Psychiatrie 10:281-283

Kapfhammer HP (1998) Internistische Aspekte der Behandlung mit Antidepressiva. In: Hewer W, Lederbogen F (Hrsg) Internistische Probleme bei psychiatrischen Erkrankungen. Enke, Stuttgart, S 51-87

Kapfhammer HP (1999) Psychopharmakotherapie depressiver Störungen bei somatischen Erkrankungen mit selektiven Serotonin-Wiederaufnahmehemmern, anderen neueren Antidepressiva und amphetaminergen Substanzen. In: Diefenbacher A (Hrsg) Aktuelle Konsiliarpsychiatrie und -psychotherapie. Thieme, Stuttgart, S 131-160

Kapfhammer HP (2001) Begleitdepression bei körperlichen Krankheiten: Epidemiologie und Diagnostik. Jatros Neurologie/Psychiatrie (Sonderpublikation): 6-17

Kapfhammer HP (2002) Organisch depressive Störungen: Koronare Herzerkrankung, Diabetes mellitus, Krebs. In: Laux G (Hrsg) Depression 2000. Springer, Berlin Heidelberg New York Tokyo, S 65-86
Kapfhammer HP, Rothenhäusler HB, Krauseneck T, Stoll C, Schelling G (2001) Posttraumatische Belastungsstörung bei Patienten mit ARDS. Jahrbuch der Medizinischen Psychologie 20. Hogrefe, Göttingern, Bern, Toronto, Seattle, S 119-138

Kathol RG, Wenzel RP (1992) Natural history of symptoms of depression and anxiety during inpatient treatment on general medicine wards. J Gen Int Med 7:287-293

Kathol RG, Turner R, Delahunt J (1986) Depression and anxiety associated with hyperthyroidism: Response to antithyroid therapy. Psychosomatics 27:501-505

Kathol RG, Mulgi A, Williams J et al. (1990) Diagnoss of major depression in cancer patients according to four stes of criteria. Am J Psychiatry 1021-1024

Katon W (1998) The effect of major depression on chronic medical illness. Semin Clin Neuropsychiatry $2: 82-86$

Katon W, Sullivan DM (1990) Depression and chronic medical illness, J Clin Psychiatry 51 (Suppl 6): 3-11

Katon W, Berg AO, Robins AJ, Risse S (1986) Depression: Medical utilization and somatization. West J Med 114:564-568

Katz IR (1996) On the inseparability of mental and physical health in aged persons. Am J Geriatr Psychiatry 4:1-6

Klein DF (1993) False suffocation alarms, spontaneous panics, and related conditions: An integrative hypothesis. Arch Gen Psychiatry $50: 306-317$

Klein E, Cnaani E, Harel T et al.(1995) Altered heart rate variability in panic disorder patients. Biol Psychiatry 37:18-24

Klerman GL (1981) Depression in the medically ill. Psychiatr Clin North Am 4:301-317

Koenig HG, Breitner JCC (1990) Use of antidepressants in medically old patients. Psychosomatics $31: 22-32$

Koenig HG, Meador KG, Cohen HJ et al. (1988) Self-rated depression scales and screening for major depression in the older hospitalized patient with medical illness. J Am Geriatr Med 36: 699-706

Koenig HG, Georage LK, Meador KG (1997) Use of antidepressants by nonpsychiatrists in the treatment of medically ill hospital ized depressed elderly patients. Am J Psychiatry 154:1369-1375

Koenig HG, Pappas P, Hoslinger T et al. (1995) Assessing diagnostic approaches to depression in medically ill older adults: How reliably can mental health professions make judgements about the cause or symptoms? J Am Geriatr Soc 43:472-478

Korff M von, Shapiro S, Burke JD et al. (1987) Anxiety and depression in a primary care clinic: Comparison of Diagnostic Interview Schedule, General Health Questionnaire, and practitioner assessments. Arch Gen Psychiatry 44:152-156

Korff M von, Ormel H, Katon W et al. (1992) Disability and depression among high utilizers of health care: A longitudinal analysis. Arch Gen Psychiatry 49:91-100

Larcombe NA, Wilson PH (1984) An evaluation of cognitive behavior therapy for depression in patients with multiple sclerosis. Br J Psychiatry 145:366-371

Leipzig RM (1990) Psychopharmacology in patients with hepatic and gastrointestinal disease. Int J Psychiatry Med 22:109-139

Lesko LM, Massie MJ, Holland J (1993) Oncology. In: Stoudemire A, Fogel (eds) Psychiatric care of the medical patient. Oxford University Press, Oxford New York Toronto, pp 565-590

Levenson H, Hales RE (1993) Brief psychodynamically informed therapy for medically ill patients. In: Stoudemire A, Fogel BS (eds) Medical-psychiatric practice, vol 2. American Psychiatric Press, Washington/DC London, pp 3-37

Levenson H, Servis M, Hales RE (2000) Brief psychodynamic therapy in the medically ill. In: Stoudemire A, Fogel BS, Green berg DB (eds) Psychiatric care of the medical patient. $2^{\text {nd }}$ edn. Oxford University Press, Oxford, pp 17-30

Levenson J (1992) Psychosocial interventions in chronic medical illness. Gen Hosp Psychiatry 14:43-49

Levenson JL, Bemis C (1991) The role of psychological factors in cancer onset and progression. Psychosomatics 32:124-132

Levenson JL, Friedel RO (1985) Major depression in patients with cardiac disease. Psychosomatics 26:91-102

Levy N (1990) Psychopharmacology in patients with renal failure. Int J Psychiatry Med 20:325-334

Liang LP, Dunn SM, Gorman A, Stuart-Harris R (1990) Identifying priorities of psychoocial need in cancer patients. Br J Cancer $62: 1000-1003$ 
Lieberman JA, Cooper TB, Suckow RW et al. (1985) Tricyclic antidepressant and metabolite levels in chronic renal failure. Clin Pharmacol Ther 37:301-307

Light RW, Merrill EJ, Despars J et al. (1986) Doxepin treatment of depressed patients with chronic obstructive pulmonary disease. Arch Intern Med 146:1377-1380

Lipowski Z (1990) Delirium: Acute confusional states. Oxford University Press, New York Oxford

Lipowski ZJ (1975) Physical illness, the patient and his environment. In: Reiser M (ed) American handbook of psychiatry, vol 4. Basic Books, New York, pp 3-42

Lipsey JR, Robinson RG, Pearlson GD et al. (1984) Nortriptyline treatment of post-stroke depression. Lancet i: 297-300

Lishman WA $\left(1987,{ }^{3} 1998\right)$ Organic psychiatry. The psychological consequences of cerebral disorder, 2nd edn. Blackwell, Abingdon

Lloyd GG, Cawley RH (1978) Psychiatric morbidity in men one week after first acute myocardial infarction. $\mathrm{Br} \mathrm{J}$ Med 2: 1453-1454

Lustman PJ, Griffith LS, Clouse RE et al. (1997) Effects of nortriptyline on depression and glycemic control in diabetes: Results of a double-blind, placebo-controlled trial. Psychosom Med $59: 241-250$

Maricle RA, Hosenpud JD, Norman DJ et al. (1989) Depression in patients being evaluated for heart transplantation. Gen Hosp Psychiatry 11:418-424

Maguire P, Haddad P (1996) Psychological reactions to physical reactions. In: Guthrie E, Creed F (eds) Seminars in liaison psychiatry. Royal College of Psychiatrists, Gaskell Glasgow, pp 157-191

Maguire GP, Julier DL, Hawton KE et al. (1974) Psychiatric morbidity and referral on two general medical wards. Br Med J: 268-270

Maguire GP, Lee EG, Bevington DJ et al. (1978) Psychiatric problems in the first year after mastectomy. Br Med J: 963-965

Manoach M, Varon D, Neumann M et al. (1989) The cardioprotective features of tricyclic antidepressants. Gen Pharmacol 20: 269-275

Masand P, Pickett P, Murray G (1991) Psychostimulants for secondary depression in medical illness. Psychosomatics 32: 203-208

Massie MJ, Holland JC (1990) Depression and the cancer patient. J Clin Psychiatry 51 (Suppl 7): 12-19

Mayeux T (1990) Depression in the patient with Parkinson's disease. J Clin Psychiatry 51:20-23

Mayou RA, Sharpe M (1995) Psychiatric illness associated with physical disease. Baillieres Clin Psychiatry 1:201-223

Mayou R, Hawton K, Feldman E (1988) What happens to medical patients with psychiatric disorder? J Psychosom Res 32:541-549

Meares RA, Mills JE, Horvath TB (1971) Amitriptyline and asthma. Med J Aust 2:25-28

Moffic HS, Paykel ES (1975) Depression in medical in-patients. Br J Psychiatry 126:346-353

Möller HJ (1996) Forschungsstandort Deutschland: Klinische Entwicklung neuer Antidepressiva. Psycho 22:147-157

Montgomery SA (1995) Safety of mirtazapine: A review. Int Clin Psychopharmacol 10 (Suppl 2): 37-45

Morris PL, Robinson RG, Andrzejewski P et al. (1993) Association of depression with 10 year post-stroke mortality. Am J Psychiatry $150: 124-129$

Morrison MF, Kastenberg JS (1997) Differentiation of secondary from primary mood dissorders: Controversies and consensus. Semin Clini Neuropsychiatry 2:232-243

Müller N, Kapfhammer HP, Spatz R, Hippius H (1995) Lithiumprophylaxe: Hohe Effizienz und geringes Risiko bei regelmäßiger Überwachung. Dtsch Ärztebl 92: A365-A369

Murray GB, Shea V (1987) Electroconvulsive therapy for poststroke depression. J Clin Psychiatry 47:258-260

Noyes R, Hoehn-Saric R (1998) The anxiety disorders. Cambridge University Press, Cambridge

Patten SB, Love EJ (1997) Drug-induced depression. Psychother Psychosom 66:63-73

Pauli P, Wiedemann G, Dengler W et al. (1999) Anxiety in patients with an automatic implantable cardioverter defibrillator: What differentiates them from panic patients? Psychosom Med 61: $69-76$
Perez-Stable WJ, Miranda J. Munoz RF et al. (1990) Depression in medical outpatients: Underrecognition and misdiagnosis. Arch Int Med 150:1983-1988

Perkins D, Evans DL (1991) HIV-related major depression. Psychosomatics 32:451-454

Popkin MK, Callies AL, Mackenzie TB (1985) The outcome of antidepressant use in the medically ill. Arch Gen Psychiatry 42: $1160-1166$

Popkin MK, Callies AL, Lentz RD et al. (1988) Prevalence of major depression, simple phobia, and other psychiatric disorders in patients with long-standing Type I diabetes mellitus. Arch Gen Psychiatry 45:64-68

Popkin MK, Callies AL, Colon EA et al. (1990) Adjustment disorders in medically ill patients referred for consultation at a university hospital. Psychosomatics 31:410-414

Prigerson HG, Shear K, Frank E et al. (1997) Traumatic grief: A case of loss-induced trauma. Am J Psychiatry 154:1003-1008

Rabkin JG, Rabkin R, Harrison W et al. (1994) Effect of imipramine on mood and enumerative measures of immune status in depressed patients with HIV infection. Am J Psychiatry 151: $516-523$

Reding MJ, Orto LA, Winter SW et al. (1986) Antidepressant therapy after stroke. Arch Neurol 43:763-765

Regan W, Margolin R, Mathew R (1989) Cardiac arrhythmia following rapid imipramine withdrawal. Biol Psychiatry 25: 482-484

Reifler BV, Teri L, Raskind M et al. (1989) Double-blind trial with imipramine in Alzheimer's disease patients with and without depression. Am J Psychiatry 146:45-49

Richardson JL, Zarnegar Z, Bisno B, Levine A (1990) Psychosocial status at initiation of cancer treatment and survival. J Psychosom Res 34:189-201

Ries RK, Gilbert DA, Katon W (1984) Tricyclic antidepressants in the therapy for peptic ulcer disease. Arch Intern Med 144:566-569

Rifkin A, Reardon G, Siris S et al. (1985) Trimipramin in physical illness with depression. J Clin Psychiatry 46:4-8

Robertson MM, Trimble MR (1985) The treatment of depression in patients with epilepsy. J Affect Disord 9:127-136

Robertson MM, Trimble MR Townsend HRA (1987) Phenomenology of depression in epilepsy. Epilepsia 28:364-372

Robbins E, Guze S (1972) Classification of affective disorders. In: Williams T, Katz M, Shields A (eds) Recent advantages in the psychobiolgy of the depressive illnesses. Government Printing Office, Washington/DC

Robinson RG, Starr LB, Kubos KL et al. (1983) A two-year longitudinal study of post-stroke mood disorders: Findings during the initial evaluation. Stroke 14:736-741

Robinson RG (1998) The clinical neuropsychiatry of stroke. Cognitive, behavioral and emotional disorders following vascular brain injury. Cambridge University Press, Cambridge

Rodin G, Voshart K (1986) Depression in the medically ill. Am J Psychiatry 143:696-705

Rodin R, Craven J, Littlefield C (1991) Depression in the medically ill. An integrated approach. Brunner/Mazel, New York

Roose SP, Glassman A, Giardina E (1987) Tricyclic antidepressants in depressed patients with cardiac conduction disease. Arch Gen Psychiatry 44:273-275

Roose SP, Laghrissi-Thode F, Kennedy JS et al. (1998) Comparison of paroxetine and nortriptyline in depressed patients with ischemic heart disease. JAMA 279:287-291

Rotzinger S, Bourin M, Akimoto Y, Coutts RT, Baker GB (1999) Metabolism of some „second“- and „fourth“-generation antidepressants: Iprindole, viloxazine, bupropion, mianserin, maprotiline, trazodone, nefazodone, and venlafaxine. Cell Mol Neurobiol 19(4):427-442

Rubey RN, Lydiard RB (1994) Psychopharmacology in the medically ill. In: Silver P (ed) Psychotropic drug use in the medically ill. Adv Psychosom Med, vol 21. Karger, Basel, pp 1-27

Saran AS (1985) Depression after minor closed head injury. J Clin Psychiatry 46:335-338

Satel SL, Nelson JC (1989) Stimulants in the treatment of depression. A critical overview. J Clin Psychiatry 50:241-249

Satlin A (1998) The comorbidity of depression and medical illness in the elderly. In: Tohen M (ed) Comorbidity in affective disorders. Dekker, New York Basel, pp 157-178 
Schag CAC, Heinrich RL (1989) Anxiety in medical situations: Adult cancer patients. J Clin Psychology 45:20-27

Schelling G, Scholl C, Haller M et al. (1998) Health-related quality of life and post-traumatic stress disorder in survivors of the acute respiratory distress syndrome (ARDS). Crit Care Med 26:651-659

Schenker S, Bergstrom R, Wolen R et al. (1988) Fluoxetine disposition and elimination in cirrhosis. Pharmacol Ther 44:353-359

Schiffer RB, Wineman NM (1990) Antidepressant pharmacotherapy of depression associated with multiple sclerosis. Am J Psychiatry 147: 1493-1497

Schiffer RB, Herndon RM, Rudick RA (1985) Treatment of pathological laughing and weeping with amitryptiline. N Engl J Med 312:1480-1482

Schmale AH (1979) Reactions to illness. Psychiatr Clin North Am $2: 321-330$

Schulberg HC, Saul M, McClelland M (1985) Assessing depression in primary medical and psychiatric practices. Arch Gen Psychiatry $12: 1164-1170$

Schüßler G (1998) Krankheitsbewältigung und Psychotherapie bei körperlichen und chronischen Erkrankungen. Psychotherapeut $43: 382-390$

Schwab JJ, McGinness NH, Marder L et al. (1966) Evaluation of anxiety in medical patients. J Chron Dis 19:1049-1057

Schwartz JA, Speed N, Clavier E (1988) Tricyclic antidepressants in treatment of the medically ill: The value of psychiatric consultation. Int J Psychiatry Med 18:235-241

Schwartz JA, Speed N, Beresford TP (1989) Antidepressants in the medically ill: Prediction of benefits. Int J Psychiatry Med 19: 363-369

Secor JW, Schenker S (1987) Drug metabolism in patients with liver disease. Adv Intern Med 32:379-406

Sellers EM, Bendayan R (1987) Pharmacokinetics of psychotropic drugs in selected patient populations. In: Meltzer HY (ed) Psychopharmacology: The third generation of progress. Raven, New York, pp 1397-1406

Shakin EJ, Holland J (1988) Depression and pancreatic cancer. J Pain Symptom Manage 3:194-198

Sharpe M, Hawton K, Seagrott V et al. (1994) Depressive disorders in long-term survivors of stroke. Br J Psychiatry 164:380-386

Sherbourne CD, Jackson CA, Meredith LS et al. (1996) Prevalence of comorbid anxiety disorders in primary care outpatients. Arch Fam Med 5:27-34

Sliman RJ, Donahue TA, Jarjoura D et al. (1992) Recognition of depression by internal medicine residents. J Community Health $17: 143-152$

Sonino N, Fava GA, Belluardo P et al. (1993) Course of depression in Cushing's syndrome: Response to treatment and comparison with Graves' disease. Horm Res 39:202-206

Spiegel D (1991) Psychosocial aspects of cancer. Curr Opin Psychiatry $4: 889-897$

Spiegel D (1994) Krebs und Depression. Verhaltenstherapie 4: $81-88$

Spiegel D, Lazar SG (1997) The need for psychotherapy in the medically ill. Psychoana Inquiry (Suppl) 17:45-50

Spiegel D, Bloom JR, Kraemer HC et al. (1989) Effect of psychological treatment on survival of patients with metastatic breast cancer. Lancet ii: $888-889$

Starkman MN, Zelnik TC, Nesse TC et al. (1985) Anxiety in patients with pheochromocytomas. Arch Intern Med 145:248-252

Starkstein SE, Robinson RG (1989) Affective disorder and cerebral vascular disease. Br J Psychiatry 154: 170-182

Starkstein SE, Preziosi TJ, Bolduc PL, Robinson RG (1990) Depression in parkinson's disease. J Nerv Ment Dis 178:27-31

Stefanek ME, Shaw A, DeGeorge D et al. (1989) Illness-related worry among cancer patients: Prevalence, severity and content. Cancer Invest 7:365-371
Stoudemire A, Atkinson P (1988) Use of cyclic antidepressants in patients with cardiac conduction disturbances. Gen Hosp Psychiatry 10:389-397

Stoudemire A, Hales RE (1991) Psychological and behavioral factors affecting medical conditions and DSM-IV. Psychosomatics $32: 5-13$

Stoudemire A, Moran MG, Fogel BS (1990) Psychotropic use in the medically ill: Part I. Psychosomatics 31:377-391

Stoudemire A, Fogel BS, Gulley LR (1991) Psychopharmacology in the medically ill: An update. In: Stoudemire A, Fogel BS (eds) Medical psychiatric practice. American Psychiatric Press, Washington/DC London, pp 29-97

Strian F, Ploog D (1988) Anxiety related to nervous system dysfunction. In: Noyes R, Roth M, Burrows GD (eds) Handbook of anxiety 2. Elsevier, Amsterdam, pp 431-475

Surman O (1991) Hemodialysis and renal transplantation. In: Cassem N (ed) Massachusetts General Hospital Handbook of General Hospital Psychiatry, 3rd edn. Mosby Year Book, St. Louis/ MO, pp 401-430

Tarter RE, Switala J, Carra J et al. (1987) Inflammatory bowel disease: Psychiatric status of patients before and after disease onset. Int J Psychiatry Med 17:173-181

Thompson WL, Thompson TL (1984) Treating depression in asthmatic patients. Psychosomatics 25:809-812

Thompson WL, Thompson TL (1993) Pulmonary disease. In: Stoudemire A, Fogel B (eds) Psychiatric care of the medical patient. Oxford University Press, New York, pp 591-610

Troy S, Schultz R, ParkerV et al. (1994) The effect of renal disease on the disposition of venlafaxine. Clin Pharmacol Ther 56:14-21

Trzepacz PT, McCue M, Klein I et al. (1988) A psychiatric and neuropsychological study of patients with untreated Graves' disease. Gen Hosp Psychiatry 10:49-55

Trzepacz PT, Brenner R, van Thiel DH (1989) A psychiatric study of 247 liver transplantation candidates. Psychosomatics 30 : 147-153

Veith RC, Raskind MA, Caldwell JH et al. (1982) Cardiovascular effects of tricyclic antidepressants in depressed patients with chronic heart disease. N Engl J Med 306:954-959

Wells KG, Golding JM, Burnham MA (1988) Psychiatric disorder in a sample of the general population with and without chronic medical conditions. Am J Psychiatry 145:976-981

Wells KB, Golding JM, Burnam MA (1989) Affective substance use, and anxiety disorders in persons with arthritis, diabetes, heart disease, high blood pressure, or chronic lung conditions. Gen Hosp Psychiatry 11:320-327

Whitlock FA (1982) Symptomatic affective disorders. Academic Press, Sydney

Wilson RCD (1974) Antiasthmatic effect of amitriptyline. Can Med Assoc J 3:212

Winokur G (1990) The concept of secondary depression and its relationship to comorbidity. Psychiatr Clin North Am 13: 567-583

Winokur G, Black DW, Nashrallah A (1988) Depressions secondary to other psychiatric disorders and medical illnesses. Am J Psychiatry $145: 233-237$

Wolfersdorf M (1993) Suizidalität bei Tumor- und anderen körperlichen Erkrankungen. In: Staab HJ, Ludwig M (Hrsg) Depression bei Tumorpatienten. Thieme, Stuttgart New York, S 49-67

Yates WR, Bowers WA (2000) Cognitive therapy in the medicalpsychiatric patient. In: Stoudemire A, Fogel BS, Greenberg DB (eds) Psychiatric care of the medical patient. $2^{\text {nd }}$ edn. Oxford University Press, Oxford, pp 51-60

Zaubler T, Katon W (1998) Panic disorder in the general medical setting. J Psychosom Res 44:25-42

Zisook S, Paulus M, Shuchter SR, Judd LL (1997) The many faces of depression following spousal bereavement. J Affect Disord 45: 85-95 
Sektion X

Persönlichkeits-

und Verhaltensstörungen 